\title{
Massively parallel spectroscopy of sources in Galactic globular clusters
}

\author{
Dissertation \\ zur Erlangung des mathematisch-naturwissenschaftlichen \\ Doktorgrades \\ „Doctor rerum naturalium" \\ der Georg-August-Universität Göttingen \\ im Promotionsprogramm Physik \\ der Georg-August University School of Science (GAUSS) \\ vorgelegt von \\ Fabian Göttgens \\ aus Hildesheim
}

Göttingen, 2021 
Betreuungsausschuss

Prof. Dr. Stefan Dreizler

Institut für Astrophysik, Georg-August-Universität Göttingen, Göttingen, Deutschland

Prof. Dr. Ansgar Reiners

Institut für Astrophysik, Georg-August-Universität Göttingen, Göttingen, Deutschland

Dr. Tim-Oliver Husser

Institut für Astrophysik, Georg-August-Universität Göttingen, Göttingen, Deutschland

Mitglieder der Prüfungskommission

Referent: Prof. Dr. Stefan Dreizler

Institut für Astrophysik, Georg-August-Universität Göttingen, Göttingen, Deutschland

Korreferent: Prof. Nate Bastian

Donostia International Physics Center, Donostia-San Sebastián, Spanien

Weitere Mitglieder der Prüfungskommission

Prof. Laura Covi

Institut für Theoretische Physik, Georg-August-Universität Göttingen, Göttingen, Deutschland

PD Dr. René Heller

Max-Planck-Institut für Sonnensystemforschung, Göttingen, Deutschland

Prof. Dr. Wolfram Kollatschny

Institut für Astrophysik, Georg-August-Universität Göttingen, Göttingen, Deutschland

Prof. Dr. Ansgar Reiners

Institut für Astrophysik, Georg-August-Universität Göttingen, Göttingen, Deutschland

Tag der mündlichen Prüfung: 8.9.2021 


\section{Abstract}

Globular clusters (GCs) consist of hundreds of thousands of stars, densely packed into a spherical shape. Not only do GC contain ordinary main sequence and red giant stars, but also the products of frequent stellar encounters, stellar remnants, and potentially intermediate-mass black holes (IMBH). But how can we find these objects hidden between thousands of other stars? With the progress in observation techniques used in astronomy, it is possible to observe individual stars in the cores of GCs, the most crowded regions. In particular, the development of large integral-field spectrographs, such as Multi-Unit Spectroscopic Explorer (MUSE) at the Very Large Telescope, and adaptive optics to correct for atmospheric distortions, enable high-resolution observations from the ground. Using these techniques, we can efficiently observe GCs and measure individual spectra of thousands of stars simultaneously. During an automated search for emission-line objects in these spectra, we detected a previously unknown nebula in M22. The spectrum of this nebula shows emission lines of hydrogen, nitrogen, and sulphur, but it does not look like a typical spectrum of a planetary nebula (PN). Not only are its spectral lines unusual, but with its small size and a low luminosity, the nebula also does not resemble any of the four known PNe in Galactic GCs, including the one in M22. The literature contains many attempts to detect a central IMBH in a Galactic GC. So far, there is no unambiguous discovery. We use data taken with the MUSE narrow-field mode with a spatial resolution comparable to the Hubble Space Telescope to analyse the motion of stars in M80. To overcome the usual problems of previous attempts, we employ both a model based on the Jeans equations and an independent $N$-body model of M80. We find two equally probable solutions with different dynamical cluster centres: One solution has its centre close to the photometric centre from the literature, and it does not need an IMBH to explain the observed stellar motions. Another solution has a centre with a small offset from the first one. Here, a central IMBH with a mass of several thousand solar masses is needed. The $N$-body models exclude the existence of many stellar-mass black holes, which could mimic the effect of an 
IMBH on the stellar motions. 


\section{ACKNOWLeDgements}

Firstly, I would like to thank Stefan Dreizler for giving me the opportunities I had and his continuous support during my $\mathrm{PhD}$ and previous phases of my studies. I would also like to thank Sebastian Kamann, Tim-Oliver Husser, and Benjamin Giesers for answering many of my questions over the years and building the data analysis system that is the basis of this work and others. I also thank all co-authors of my works, especially Holger Baumgardt and Peter Weilbacher, for providing their expertise. I would also like to thank the many people I met at the institute, in particular Wolfram Kollatschny and Rick Hessman for the activities at conferences and during an excursion. Special thanks go to Sofia Gallego and Kaspar Schmidt for the wonderful time during our observations in Chile. Finally, I would like to thank the many authors, actors, and artists whose work inspires many people by showing us what our common future could look like. 


\section{Contents}

$\begin{array}{lll}1 & \text { Introduction } & 1\end{array}$

1.1 Evolution of astronomical observation techniques . . . . . . . 3

1.1.1 History and future of telescopes . . . . . . . . . . . 3

1.1 .2 Stellar spectroscopy $\ldots \ldots \ldots \ldots$. . . . . . . . . . . . 4

1.1.3 Multi-object and integral-field spectroscopy . . . . . . 6

$1.1 .4 \quad$ Basics of adaptive optics . . . . . . . . . . . . 7

1.1 .5 The MUSE adaptive optics system . . . . . . . . . . . 8

1.1.6 The MUSE Globular Cluster Survey . . . . . . . . . . . 8

1.2 Globular clusters . . . . . . . . . . . . . . . . . . . 9

1.2.1 The globular cluster system of the Milky Way . . . . . 9

1.2 .2 Origin of Galactic globular clusters. . . . . . . . . . . 11

1.2 .3 Globular cluster formation and evolution . . . . . . . . . 12

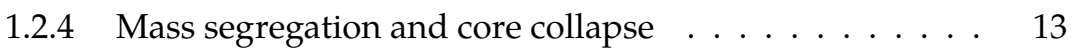

1.2 .5 The contents of old globular clusters . . . . . . . . . . . . 14

$1.2 .6 \quad$ Multiple populations . . . . . . . . . . . . . 17

1.3 Modelling globular clusters $\ldots \ldots \ldots \ldots$

1.3 .1 Observing the kinematics of globular clusters . . . . . . 18

1.3 .2 Analysing motions in globular clusters . . . . . . . . 20

1.3 .3 Jeans models of globular cluster kinematics. . . . . . . . 21

1.4 Hypothetical IMBHs in GCs . . . . . . . . . . . . . . . 26

1.4 .1 Detection methods . . . . . . . . . . . . . . . . . 26

1.4 .2 Extra-galactic IMBH candidates. . . . . . . . . . . . 27

1.4 .3 IMBH Candidates in Galactic GCs . . . . . . . . . . . 28

1.4 .4 Formation scenarios . . . . . . . . . . . . . . . . . . . . . 29

1.5 Aims of his work . . . . . . . . . . . . . . . . . . . . 30

2 Peer-reviewed journal publications 31

$2.1 \quad$ Discovery of an Old Nova Remnant in the Galactic Globular

Cluster M22 (Göttgens et al.|2019a)] . . . . . . . . . . . . . . 31 
2.2 Central kinematics of the Galactic globular cluster M80 (Göttgens

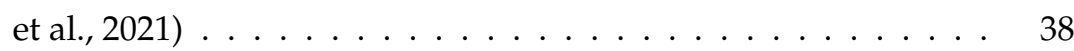

3 Additions to emission-line analysis $\quad 55$

4 Conclusions and outlook

4.1 The small emission nebula in M22 . . . . . . . . . . . . . . . 57

4.2 Is there an IMBH in M80? . . . . . . . . . . . . . . . . . 57

4.3 Central high-velocity stars in GCs . . . . . . . . . . . . . . 58

4.4 Proper-motions from MUSE NFM observations. . . . . . . . . . 59

4.5 Future observations with MUSE and BlueMUSE . . . . . . . . . 62

\begin{tabular}{ll}
\hline A Appendix & 75
\end{tabular}

A.1 A Stellar Census in Globular Clusters with MUSE: A Spectral Catalogue of Emission-Line Sources (Göttgens et al. [2019b)] . . 75 


\section{Chapter 1}

\section{INTRODUCTION}

In the large-scale order of objects humans have discovered in the universe so far, globular clusters (GCs) sit between open clusters with several thousand stars and galaxies which contain billions of stars. At this position, GCs consist of several hundred thousand to a million gravitationally bound stars, which form a roughly spherical collection with a typical diameter of several parsecs. Globular clusters are found in the haloes of galaxies, including about 160 of them in the Milky Way, where they are on orbits around the centre of their host galaxy. The spatial density of stars in a GC is much higher compared to the solar neighbourhood and among the highest found in the universe. Historically, astronomical observations of individual stars in the dense core of GCs were therefore difficult tasks. Today, with large telescopes, efficient instruments, electronic detectors, and systems to correct for the effects of atmospheric turbulence, it is possible to observe stars in the centres of GCs. Not only is it now possible to measure their positions and brightnesses but also their spectra, allowing us to determine stellar velocities and physical properties of their atmosphere. Figure 1.1 shows the large Galactic GC NGC 104 (47 Tuc). The stellar density increases from the outskirts of the cluster toward the centre where individual stars are no longer visible due to crowding. This illustrates the difficulty in observing GCs, especially their dense cores, where the stellar density $10^{4.4} \mathrm{M}_{\odot} \mathrm{pc}^{-3}$ in the case of NGC 104 (Baumgardt \& Hilker. 2018). While Section 1.2 describes more properties of Galactic GCs, I will first discuss the evolution of the observing techniques that enabled astronomers to analyse GCs and derive their characteristics that will be important for the remainder of this work. 


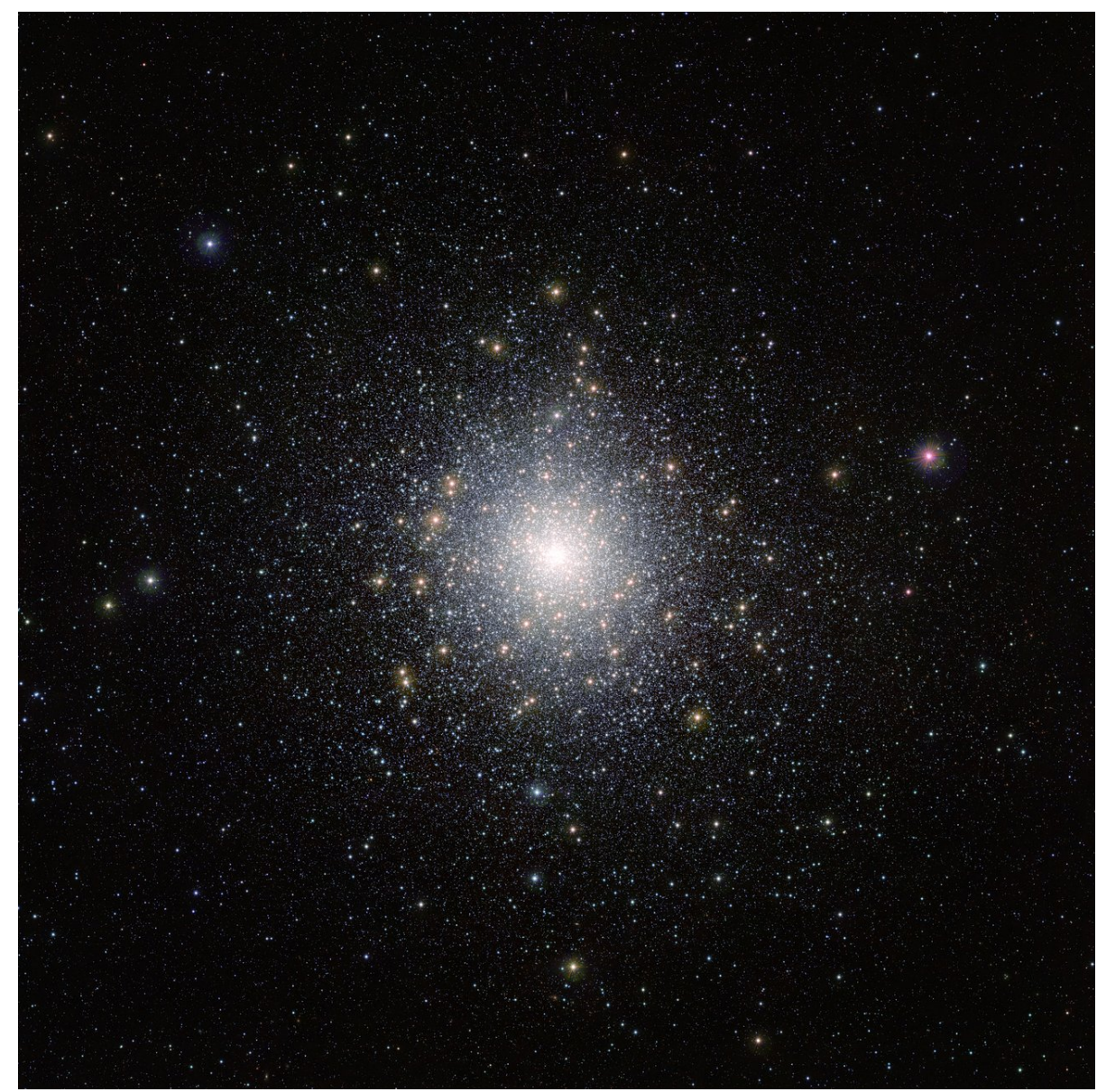

Figure 1.1: The Galactic globular cluster NGC 104. This image was combined using three infrared images taken with VISTA. The field of view is $46 \mathrm{arcmin}$ by 46 arcmin (ESO, 2013). Credit: ESO/M.-R. Cioni/VISTA Magellanic Cloud survey. Acknowledgement: Cambridge Astronomical Survey Unit. License: https://creativecommons.org/licenses/by/4.0/ 


\subsection{Evolution of astronomical observation techniques}

There has been huge progress in the observational techniques of astronomy in the last two hundred years. This progress is visible in the design of telescopes, the construction of instruments fed with the light of these telescopes, and the analytical tools that are used to derive results from the data.

\subsubsection{History and future of telescopes}

Professionally used telescopes went from the small refractors of Galileo, who invented the telescope around 1600, and single-mirror reflecting telescopes used by Herschel and others in the 19th century, to adaptive-optics assisted single-mirror (e.g. ESO's Very Large Telescope, VLT, Wilson, 1999, p.205) and multi-mirror telescopes such as the Large Binocular Telescope (Wilson. 1999. p.190) to segmented-mirror reflecting telescopes (Keck telescopes, Wilson, 1999, p.205) and to space-based reflecting telescopes such as the Hubble Space Telescope (HST, Wilson, 1999, p.193), which were all designed and constructed at the end of the 20th century. During this evolution, the size of the light-collecting area, i.e. lens diameter for refractors and the mirror diameter in case of reflectors, increased by slightly more than two orders of magnitude: from a few centimetres at the beginning of the 17th century to about 10 metres until the end of the 20th century. As not only the size of the light-collecting area increased but also the sensitivity of the light-detecting instruments and the possible exposure time, the overall improvement in astronomical observations is much larger than two orders of magnitude. Until the end of the 19th century, astronomers had only one way to conduct observations and to record them: to see through the telescope with their own eyes and make drawings of what they saw. After the invention of photographic techniques in the mid-19th century, astronomers began to take photographs of the Moon, the Sun, Vega, and the Great Comet of 1882 (Graham-Smith, 2016, p.19). In contrast to naked-eye observations, photographic observations could be done over an extended period of time, while collecting light from the astronomical source as long as it could be reliably centred on the same spot on the photographic medium. Increasing the focal length and thus the magnification of telescopes became less important after the introduction of photography because the photographic plates could record details on small spatial scales much better than the human eye. This is in contrast to the diameter of the telescope mirror which continues to increase until today (Graham-Smith, 2016, p.19). Modern telescopes are equipped with even more efficient detectors made of light-sensitive semiconducting materials, charge-coupled devices (CCD), that can be read out electronically. 
The first observation of a globular cluster was probably made by Ihle in 1665 when he observed M22 (NGC 6656, Steinicke, 2017, p.16). Later observations were made by Halley, Messier, and Herschel (Steinicke, 2017, p.16). It was Herschel who first named this spherical collection of stars a 'globular cluster'. The catalogue of Messier, still in use today, already contained among galaxies and open clusters 29 globular clusters (Stoyan et al., 2008, p.55). The later 'General Catalogue of Nebulae and Clusters' of Herschel, the precursor of the widely-used New General Catalogue (NGC), contains 38 objects that are today known as GCs (Steinicke, 2017, p.32). As photography was not yet invented, we have only sketches, written articles, and catalogues as proof or records of the observations of Ihle, Messier and Herschel.

The future of ground-based optical observations will be several extremely large telescopes with apertures of 25 to $40 \mathrm{~m}$ which are currently under construction and are expected to see first light in the mid- to late-2020s: the Giant Magellan Telescope, the Thirty Meter Telescope, and ESO's Extremely Large Telescope (Gilmozzi \& Spyromilio, 2007: Graham-Smith, 2016, p.215). Just as the telescopes in the previous generation, these are built in Chile and on Hawaii, where the light pollution is negligible, the atmospheric turbulence is low, and existing facilities are close by.

In space, the successor of HST will be the James Webb Space Telescope (JWST) equipped with a $6.5 \mathrm{~m}$ main mirror, as well as photometric and spectroscopic instruments. In contrast to HST, JWST instruments will only be sensitive to red to mid-infrared (600 nanometres to 28 micrometres) wavelengths (Gardner et al. 2006). Like many large-scale public projects, JWST has a history of delays and its launch, planned for 2007 in 1998 (Lilly, 1998), is currently planned for October 2021.

\subsubsection{Stellar spectroscopy}

Stellar spectroscopy is the astronomical tool that allows us to determine the composition of a stellar atmosphere among other properties.

Historically, Newton discovered in the 17th century that the light from the Sun can be split with a prism into the colours of the rainbow, which he combined again into white light by adding another prism (Hearnshaw, 2014. p.15). Joseph Fraunhofer was the first astronomer to study the light of the Sun and a few bright stars using spectroscopy. To magnify the spectra, he added a small telescope after the prism (Hearnshaw, 2014, p.18). He used this spectroscope to discover dark lines in the otherwise bright solar spectrum, an observation that was already made about 10 years earlier by William Hyde Wollaston (Hearnshaw, 2014, p.16). Fraunhofer labelled these absorption lines, 
the Fraunhofer lines, using Roman letters . He later observed the spectra of the Moon, Mars, and Venus (Hearnshaw, 2014, p.18). Kirchhoff and Bunsen investigated in laboratory work which emission lines are caused by different chemical elements, and Kirchhoff produced a catalogue of the solar absorption lines and the responsible element (Hearnshaw, 2014, p.29). In the 1860s, Anders Ångström and others observed the Fraunhofer lines with improved gratings intending to produce accurate wavelengths for the solar absorption lines. Henry Rowland achieved the best wavelength precision at that time of $0.001 \mathrm{~nm}$ with a curved grating (Hearnshaw, 2014, p.2). Roughly at the same time, William Huggins, Angelo Secci, and others observed the spectra of bright stars and found the same elements as in the Sun. These observations were still done with one or multiple prisms as the diffractive element (Hearnshaw, 2014, p.43). Henry Draper and Willian Huggins introduced photographic plates into stellar spectroscopy (Hearnshaw, 2014, p.45). The work of Henry Draper and others resulted in the Henry Draper (HD) catalogue published in the early 20th century which (including its extension) contained spectra of 360,000 stars (Hearnshaw. 2014. p.83). It used a stellar classification based on the spectral lines present or absent in the spectrum, the Harvard classification, developed and applied by Annie Jump Cannon (Hearnshaw, 2014, p.71). Another development in the instrumentation for stellar spectroscopy was the invention of the Coudé configuration for telescopes which directed the light to a fixed focus point so that larger and heavier spectrographs could be placed in a separate room (Hearnshaw, 2014, p.6). Gratings, in particular efficient blazed gratings, started to replace prisms as the diffractive element around 1930 (Hearnshaw, 2014. p.8). Echelle spectrographs that use a cross-dispersing element to produce a stacked set of spectra instead of a single long strip were invented in 1949 by George Harrison (Hearnshaw, 2014, p.9). This type of spectrograph is especially useful in combination with a typically quadratic CCD sensor. Many modern spectrographs are of the echelle type, including the ESO instruments HARPS (Mayor et al. 2003), and ESPRESSO (Pepe et al. 2014).

Besides stellar classification, stellar spectra were used to measure the radial velocities of stars and for spectral analysis. The radial velocity $v$ of a star changes the wavelengths $\lambda$ of spectral lines relative to the laboratory wavelength and this Doppler shift $\Delta \lambda$ can be measured and converted into a velocity: $v=c \Delta \lambda / \lambda$. Radial velocity surveys of globular clusters are discussed in Section 1.3.1. Quantitative spectral analysis, i.e. determination of element abundances, was only possible after advances in theoretical physics, especially in the theory of atoms and ionisation, and also in laboratory physics to measure the required properties of elements (Hearnshaw, 2010). 


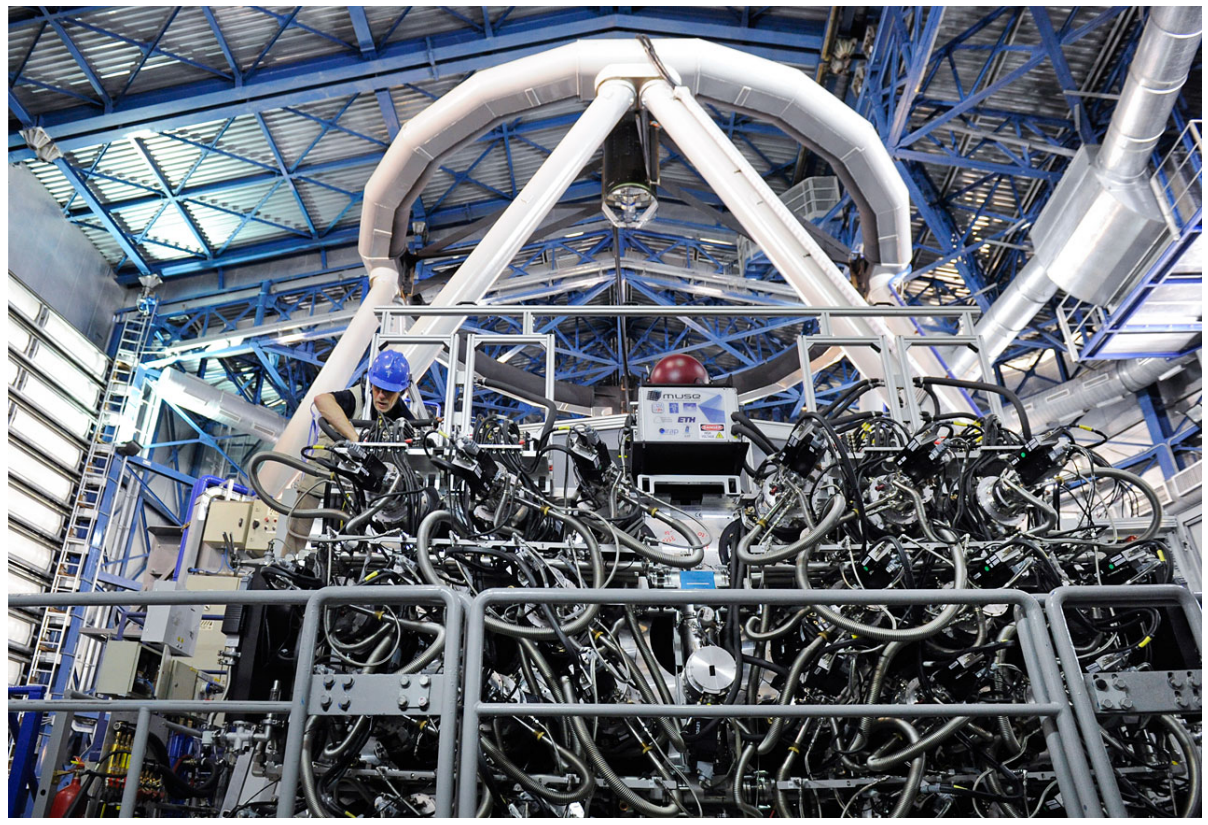

Figure 1.2: The integral-field spectrograph MUSE in front of the UT4 telescope. The visible hoses are part of the cooling system. (ESO, 2015). Credit: ESO License: https://creativecommons .org/licenses/by/4.0/

\subsubsection{Multi-object and integral-field spectroscopy}

In the case of dense stellar fields such as globular clusters or galaxy clusters, taking the spectrum of a single star or galaxy one after another is a waste of telescope time. Multi-object spectrographs can observe the spectra of several sources at the same time, increasing the efficiency. For example, fibre-fed multi-object spectrographs use optical fibres positioned in the focal plane at the location of the selected sources which direct the light side-by-side into a usual long-slit spectrograph. The resulting exposure contains the individual spectra next to each other (Bacon \& Monnet, 2017, p.70).

An integral-field spectrograph (IFS) is an extension of the concept of multiobject spectrographs. An IFS produces a whole spectrum for each pixel in its field of view (FoV). The final data product is not a two-dimensional image of a spectrum but a three-dimensional datacube with one spectral axis and two spatial axes (Bacon \& Monnet, 2017, p.95). In the case of the Multi-Unit Spectroscopic Explorer (MUSE, Bacon et al. 2010, see Fig. 1.2) at the VLT, the FoV has a size of $1 \operatorname{arcmin}^{2}$ that is sliced by mirrors into 24 strips that are directed into individual spectrographs. In front of each spectrograph, another image slicer divides the incoming light into 48 slices that are then dispersed inside the spectrograph. With 3600 spectral pixels covering the range from 
4750 to $9350 \AA$ and 100,000 spatial pixels, the instrument has about 360 million pixels in total. The advantage of integral-field spectrographs such as MUSE compared to multi-object spectrographs is that the FoV is sampled without gaps, which avoids losing stars in the gaps, especially in the crowded cores of GCs. Disadvantages are the smaller FoV diameters in the order of arcseconds to 1 arcminute, and a lower spectral resolution (Bacon \& Monnet, 2017, p. 133). Given the high stellar density in globular clusters, a single observation with MUSE of a nearby GC contains the spectra of several thousand stars, see Section 1.1.6.

\subsubsection{Basics of adaptive optics}

Adaptive optics is the technique that increases the image quality by correcting for atmospheric distortion. Turbulent motion of air pockets with different temperatures cause spatial and temporal variations of the refractive index, distorting the wavefronts of stellar light going through the atmosphere (Davies \& Kasper. 2012: Rigaut \& Neichel. 2018). This distortion is compensated in realtime by e.g. a deformable mirror in the telescope optics (Tyson. 2011. chapter 6). To determine the optimal shape, the deformable mirror, the adaptive optics system of a telescope measures the wavefronts received from a single or multiple bright point sources (guide stars) close to the observation target. The wavefront measurements are then used to bring the deformable mirror into a shape that removes the distortions. Ideally, the light from the guide stars experiences the same distortions as the light from the target object and the new shape of the deformable mirror also corrects distortions in the wavefronts of the light for the target object. The guide stars can be natural, e.g. stars or compact astronomical sources, or they can be artificially created using lasers. Since the guide stars need to be bright enough to allow wavefront measurements, not all stars can be used. This limits the area on the sky for AO observations where the target object can be located to less than about one per cent of the whole sky. In contrast, artificial guide stars can always be produced where the telescope is pointing towards and, since an additional less bright star is typically still needed, the sky coverage is much better (about 30 to 70 per cent, Rigaut \& Neichel 2018). Systems with multiple laser guide stars have a larger FoV where the AO system improves the image quality compared to systems with only one laser guide star. Many variants of $\mathrm{AO}$ systems exist, with differences in their complexity and capability. In general, a system that corrects over a large FoV will have a lower increase in image quality compared to a system that covers a small FoV (see Fig. 2 in Rigaut \& Neichel. 2018).

All extremely large telescopes will be equipped with AO to achieve diffraction- 
limited observations, at least for some instrument modes. Most planned instruments at the extremely large telescopes are expected to work with wavelengths from the red to near-infrared, as the $\mathrm{AO}$ correction is more effective for these wavelengths (see e.g. Busoni et al., 2020).

\subsubsection{The MUSE adaptive optics system}

UT4 at the VLT is equipped with an adaptive optics system that includes a deformable secondary mirror, four lasers to create artificial guide stars, and an $\mathrm{AO}$ module specific for each AO-supported instrument (e.g. GALACSI for MUSE, Stuik et al., 2006; Ströbele et al., 2012). The four sodium lasers excite atoms in a small volume in the atmospheric sodium layer at a height of about $90 \mathrm{~km}$, the light they emit is used by the wavefront sensors.

Depending on the configuration of the adaptive optics system, the size of the area with $\mathrm{AO}$ correction and the magnitude of the correction differs. For example, MUSE (Bacon et al. 2010) has two AO modes: the wide-field mode (WFM) and the narrow-field mode (NFM), see Stuik et al.(2006); Ströbele et al. (2012). When observing in the WFM, the FoV is $1 \mathrm{arcmin}^{2}$ and the AO correction decreases the FWHM of point sources to about 0.3 arcsec, even when the atmospheric seeing is about 1 arcsec. Compared to observations without $\mathrm{AO}$, the optical path contains a spectral filter to block the sodium emission from the very bright lasers. In NFM observations, the four laser guide stars are much close to the centre of the FoV. The size of the FoV is now smaller (7.5 arcsec by 7.5 arcsec), but the FWHM is much lower, typically about 60 to 80 mas (e.g. Haffert et al., 2019: Kollatschny et al., 2020) but it can increase to 150 mas under bad conditions (Xie et al. 2020). Compared to the WFM, the shape of a point source at a given wavelength, the point spread function (PSF), has a more complex shape in NFM observations. While the WFM PSF can be accurately modelled using a Moffat function, the NFM PSF has to include a model for the uncorrected spatial frequencies and for atmospheric residuals (Fétick et al. 2019). Figure 1 in Section 2.2 demonstrates that the quality of a white-light image created from a MUSE NFM datacube of GC core can be better than that of an HST ACS/WFC image.

\subsubsection{The MUSE Globular Cluster Survey}

The MUSE GC survey is an ongoing survey of Galactic and nearby extra-galactic GCs. Since starting in 2014, 33 GCs have been observed usually more than once with the integral-field spectrograph MUSE at the VLT. These GCs were selected based on their high central velocity dispersion and their relatively small heliocentric distance. After extracting a spectrum for each star from the MUSE 
datacube using Pampelmuse (Kamann et al. 2013), each spectrum is analysed with a full-spectrum fit against a grid of PhoENix models (Husser et al., 2013). The data analysis is described in detail in Kamann et al. (2018). The spectrum fit results in several stellar parameters and an estimate of the associated uncertainties, including the effective temperature of the star, its metallicity measured as $[\mathrm{M} / \mathrm{H}]$, and a radial velocity. For many stars, multiple extracted spectra and fit results are available because the GC observation was repeated after a while, or the star is located in a region in the GC where different MUSE pointings overlap. The number of observations for a given star is particularly high if both conditions are fulfilled. Assuming that multiple spectra and fit results exist, both the spectra and the fit results can be averaged to produce data with a higher signal-to-noise ratio or lower uncertainties, respectively. If multiple radial velocities are available, a time series can be computed to check for radial velocity variations that could indicate binarity or pulsations, see Section 1.3.1.

\subsection{Globular clusters}

\subsubsection{The globular cluster system of the Milky Way}

Current catalogues of Galactic globular clusters contain about 160 GCs (Baumgardt \& Hilker, 2018, Harris, 1996, 2010 version). Even more than two hundred years since the first systematic observations of GCs by Herschel, it is expected that the current list of clusters is not complete due to observational difficulties: In the Galactic plane, interstellar extinction complicates the observations of stars and clusters which leads to fewer known GCs on the far side of the Galactic centre (Ryu \& Lee, 2018). Globular clusters with large heliocentric distances far outside the Galactic plane are also hard to find because of their apparent faintness (Webb \& Carlberg, 2021). Due to the ongoing search for GCs and the uncertain classification of some objects, the GC lists in the often used catalogues of Baumgardt \& Hilker (2018) and Harris (1996, 2010 version) are not identical and possibly not up-to-date. Estimates of the number of undetected GCs range for a few GCs in the Galactic halo (Webb \& Carlberg, 2021. Contenta et al., 2017) to about 30 on the far side of the Galactic centre (Ryu \& Lee, 2018). As ultra-faint dwarf galaxies have similar absolute magnitudes to GCs, kinematic observations of new candidate GCs are needed to check if the dark matter content is low to confirm the classification as a GC (e.g. Voggel et al. 2016).

Figure 1.3 shows the locations of the GCs listed in the Harris (1996, 2010 version) catalogue in the Galactic context. Not all known GCs are plotted due to the large galactocentric distances of some of them. The large grey circles 


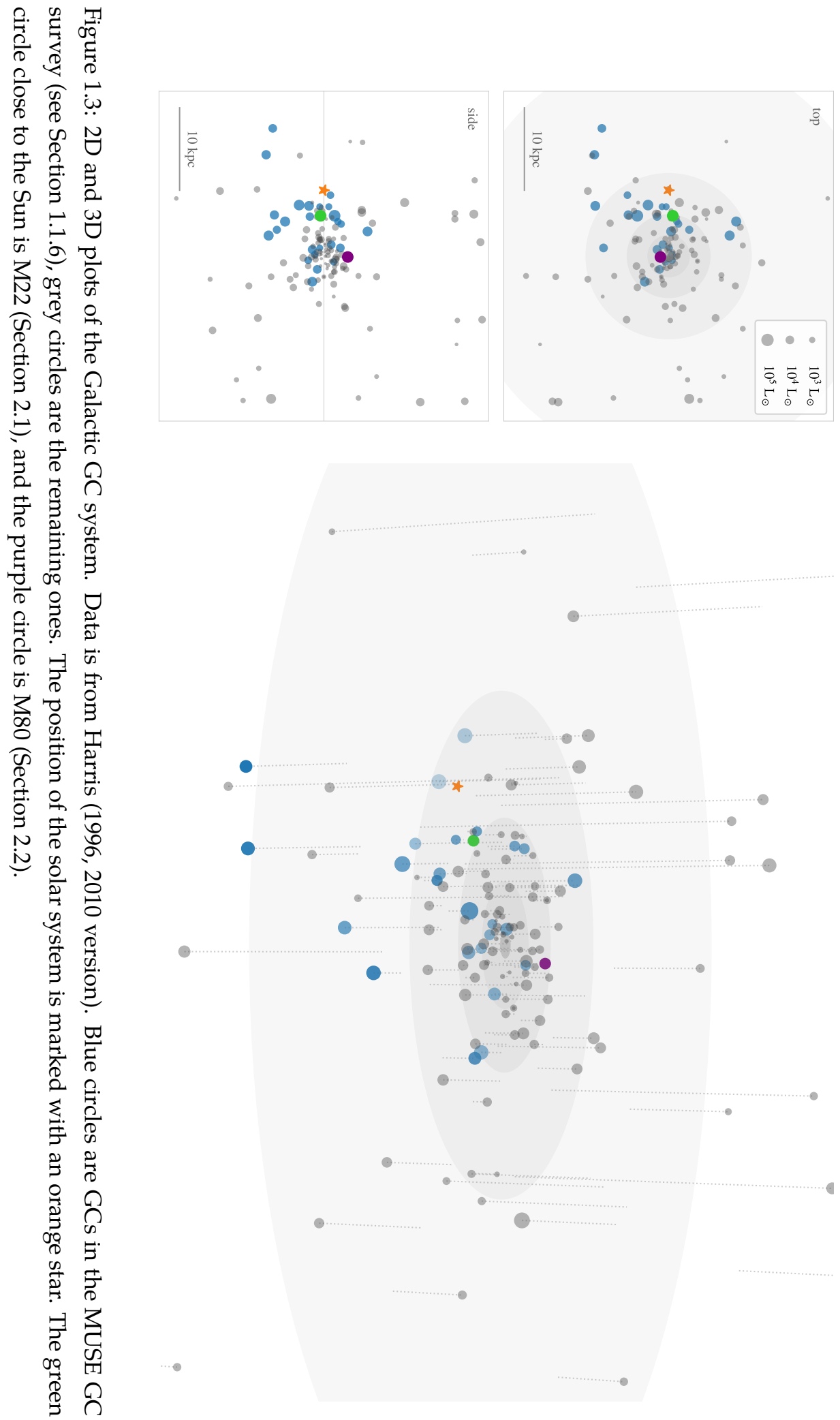


indicate the position of the Galactic disk. Blue circles are GCs observed as part of the ongoing MUSE GC survey (Section 1.1.6) and grey circles are the remaining GCs. The size of the circle indicates the luminosity of each GC. The location of the Sun is indicated with an orange star. In these plots, the higher concentration of GCs close to the Galactic centre can be easily seen. It is also apparent that the number of known GCs on the other side of the Galactic centre is lower than on this side.

The following statistics about the Galactic GC system are taken from the catalogue of Baumgardt \& Hilker (2018): Galactic GCs are located at galactocentric distances of 0.7 (NGC 6528) to about $147 \mathrm{kpc}$ (Crater). Although the range in distances to the Galactic centre is large, the median galactocentric distance is only $5.5 \mathrm{kpc}$ and 90 per cent of all clusters are within $25 \mathrm{kpc}$. The orbital periods of the GCs range from a few million years to a few billion years while most GCs have a period of about 50 to 150 million years (Bajkova \& Bobylev, 2020). The median mass of the Galactic GCs is about $10^{5}$ solar masses and it goes up to about $3.6 \times 10^{6}$ solar masses for NGC 5139. The half-light radii of GCs range from 1 to about $30 \mathrm{pc}$ and it is usually around a few parsecs for most clusters.

The ages of Galactic globular clusters range from 10 to 13 billion years (VandenBerg et al. 2013). With these ages, they belong to the oldest structures in the universe, and their ages provide a lower limit on the age of the universe (Valcin et al., 2020). The metallicity is closely linked to the age of a GC. It describes the logarithmic ratio of metals (in the astrophysical sense, i.e. every element except hydrogen and helium) to hydrogen relative to the Sun. As the metal content of the universe at the formation time of Galactic GCs 10 to 13 billion years ago was very low, Galactic GCs still have a low metallicity when compared to stars in the Galactic field. Mean metallicities of Galactic GCs range from about -2 to about -0.2 (slightly less than solar, i.e. 0 ), although values between -2 and -1 are more common than near-solar metallicities (Harris, 1996. 2010 version). All stars in a GC have roughly the same overall metallicity but the abundances of particular light elements seem to vary in each GC, causing the phenomenon of multiple populations, see Section 1.2.6.

\subsubsection{Origin of Galactic globular clusters}

The analysis of GC ages and the average stellar metallicity implies the existence of two different age-metallicity relations in the MW GC system, which has been used to divide the GC population into two subgroups: the in-situ formed GCs and the accreted GCs, which formed in galaxies that merged with the MW in the past. By using kinematic properties of GCs as determined from Gaia data, the current age, the average metallicity and the positions of the GC, 
several authors assign individual GCs to progenitor galaxies. Some kinematic quantities measurable today do not depend on time and thus will be similar for GCs from the same progenitor galaxy. They can be used to identify groups of GCs originally belonging to the same galaxy (Massari et al., 2019; Forbes, 2020: Kruijssen et al. 2020). For example, Forbes (2020) finds that NGC 1851 could be the former nucleus of Gaia-Enceladus, the most massive identified galaxy accreted by MW. Since many galaxies are thought to host nuclear star clusters (Neumayer et al., 2020), it can be expected that galaxies accreted by the Milky Way also contained nuclear star clusters. As they have similar properties as GCs and potentially similar orbits, they can be misclassified as GCs. Nuclear star clusters can be identified by their more complex metallicity distribution (Bekki \& Freeman, 2003). Similarly to NGC 1851, NGC 5139 could be the nucleus of the former Sequoia satellite galaxy (but also see Yeh et al. 2020). NGC 6093, the GC analysed in Section 2.2, is found to be an accreted GC, its original host was the massive Koala satellite (Forbes. 2020, which might be the Kraken in Kruijssen et al. 2020). In total, about 35 to 40 per cent of all Galactic GCs are associated with Galactic mergers, i.e. they were formed in a different galaxy (Massari et al. 2019: Kruijssen et al. 2019). These identifications lead to estimates of the satellite mass and the time of its merger with the Milky Way and thereby help to understand the assembly history of our Galaxy (e.g. Kruijssen et al. 2019).

\subsubsection{Globular cluster formation and evolution}

There are still open questions about when, where and how GCs formed (see the recent review of Forbes et al. 2018). The observed old ages of Milky Way GCs of about 10 to $13 \mathrm{Gyr}$ correspond to redshifts between 2 and 6, but the age uncertainties of about $1 \mathrm{Gyr}$ are too large to determine the precise redshift and thus the environment during GC formation. The formation of GCs may require a special environment, i.e. they could only form in the universe at a high redshift. GC formation models of this kind make use of e.g. merging dark matter mini-haloes (Trenti et al. 2015) or of reionization (Cen, 2001). But if young massive clusters (YMCs) are indeed young analogues of old GCs, the GC formation process must also work in the conditions found in the universe today. These models can explain YMC formation and GC formation at the same time. They treat GC formation as a byproduct of regular star formation, amplified by high gas densities and pressures that were common in the disks of spiral galaxies at $z \approx 2$ (see Section 6 in Forbes et al. 2018).

A recent example of such a model is the two-phase model of Kruijssen(2015), which is briefly summarized here: During the first phase of GC formation, initial stellar clusters from hierarchically in giant molecular clouds that exist in 
the disks of galaxies at $z>2$. In these clouds, the gas pressure is high enough to favour the build-up of clusters as opposed to single stars. This process occurs over a time span of several million years. These initial clusters will merge with other clusters in the same GMC to build larger clusters over a longer time span. Although a high gas pressure in the disk is needed to start forming clusters, it can lead to the destruction of the cluster. Its lifespan would be very short if it stayed in the disk and it is unlikely that the cluster survives here for the next approx. 10 Gyr. This disruption is due to tidal perturbation caused by density peaks in the interstellar medium in the disk. Less massive clusters are more likely to be disrupted. The cluster can be pushed into the far less dense halo of its host galaxy if the host galaxy merges with another one. Although these events are rare in the current universe, galaxy merges happened far more frequently at $z=2$. Once the cluster is in the galactic halo, the second phase starts. During this phase, the cluster loses stars at a much lower rate than before. The main processes are evaporation when interactions inside the cluster lead to the acceleration of stars which leave the cluster, and tidal interaction with the host galaxy. According to Kruijssen (2015), this model can explain many properties of observed GC systems, including the distribution of the current masses. This model includes the possibility of forming GCs today, but because the gas pressures are much lower today than in the early universe it is more unlikely. As galaxies continue to merge, their GC systems will also merge, leading to a mixed population of GCs observable today in the Milky Way.

Observationally, more precise age estimates for Milky Way GCs and thus more precise redshifts could constrain in which environment GCs form and rule out models that require different environments.

\subsubsection{Mass segregation and core collapse}

About 20 per cent of all Galactic GCs have a power-law like increase in their surface brightness profile toward their centre. This is in contrast to the remaining 80 per cent that show a flat central profile. Clusters in the first group are called core-collapsed and they are in a different dynamical state than the others. This can be explained by considering random encounters between stars in a GC, which are thought to equalise their kinetic energy. As a result, heavier stars will be decelerated and sink toward the GC centre while lighter stars get faster and move outward (Binney \& Tremaine, 2008, p.555). After many stellar encounters, this effect called mass segregation leads to a higher average stellar mass at lower distances to the GC centre. Due to the high stellar density in GCs, stars can frequently encounter others and change their velocity. After many such encounters, there will be no trace of their initial kinetic energy left. 


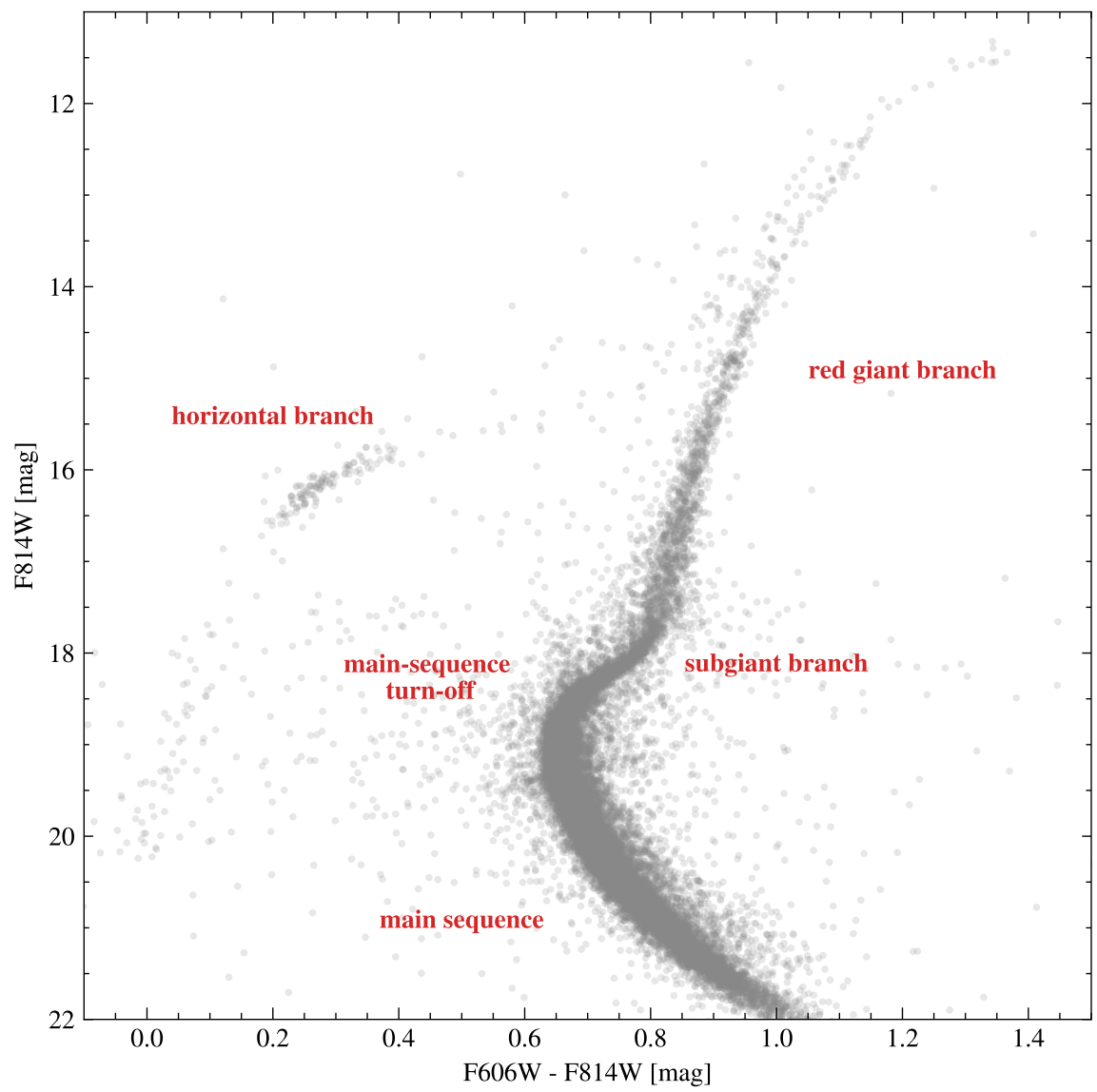

Figure 1.4: Colour-magnitude diagram (CMD) of NGC 6093 with labels indicating its main features. The photometric data is from the HST ACS survey of GCs (Anderson et al., 2008: Sarajedini et al. 2007).

This process is called relaxation. Over time, the core of a GC will lose kinetic energy to the outer regions, causing a contraction of the core, thereby leading to more frequent encounters and accelerating this process called core collapse (Binney \& Tremaine, 2008, p.614).

\subsubsection{The contents of old globular clusters}

Most stars in Galactic GCs have masses below 1 solar mass because more massive stars have already evolved into white dwarfs, neutron stars, or black holes. Since stars in a given GC have the same age and roughly the same metallicity, their colour and luminosity are determined by their initial mass. Stars with initial masses below about 0.8 solar masses are burning hydrogen in 
their cores and form the main sequence in a colour-magnitude diagram (CMD). See Figure 1.4 for a CMD of NGC 6093 as an example. Stars located at the point between main sequence and subgiant branch, the main-sequence turn-off, are the hottest stars on the main sequence. Stars on the subgiant branch and red giant branch had a higher initial mass and burn hydrogen in a shell while their core consists of helium. They have a larger radius and a higher luminosity than main-sequence stars. The hottest stars in a GC are found on the horizontal branch where stars are powered by helium fusion in their cores (Salaris \& Cassisi, 2005, Section 9).

The high stellar density inside GCs increases the probability of interactions between stars, producing several exotic objects such as blue and red stragglers, compact and interacting binary systems including cataclysmic variables, millisecond pulsars, and accreting and non-accreting stellar-mass black holes.

The large area covered with MUSE observations combined with the full spectral information make the data set an interesting place to search for the unexpected and stellar exotica. In the case of very old and inactive GC stars, emission lines are certainly a sign of rare processes or stellar properties. Göttgens et al. (2019b, see Chapter 3) searched all available extracted spectra for signs of emission lines and cross-referenced detections with existing catalogues of stellar exotica including X-ray sources and variable stars (pulsating, interacting, etc.). The following paragraphs state of few key facts about the most relevant objects classes of the work presented in this thesis.

Planetary nebulae (PNe) inside Galactic disks are former outer layers of a low-mass star on the asymptotic giant branch. If the remaining star is hot enough, its UV flux can illuminate the gas around it and create a visible nebula. The typical lifetime of a PN is several thousand years until the gas dissipated, decreasing its density and luminosity below detection. PNe exist in GCs but their number is far lower than in the Milky Way. There are only four known PNe in the Galactic GC system: Ps 1 (Pease, 1928), GJJC-1 (Gillett et al. 1989), JaFu-1, and JaFu-2 (both Jacoby et al. 1997). As the masses of AGB stars inside GCs are not large enough to create the necessary UV flux and illuminate any expelled gas shells, there must be another formation mechanism at work (Bond, 2015). In GCs, the formation of PNe could be related to binary phenomena (mass transfer, common envelopes, or even stellar mergers), but the low number of known PNe prohibits definitive conclusions (Bond, 2015: Jacoby et al. 2017). Due to their low brightnesses, the search for PNe inside the GC systems of other galaxies is difficult (Jacoby et al., 2013; Bond, 2015). Using our MUSE data and an emission-line search based on matched filtering, we found a small emission nebula in M22 which could be related to the known PN in this cluster (Göttgens 
et al. 2019a, see Section 2.1. However, we did not find any additional typical PN in our survey (Göttgens et al. 2019b, see Chapter 3).

Cataclysmic variables (CVs) and novae CVs are interacting binary systems consisting of a white dwarf with a main-sequence companion. Due to their low periods of several hours, the two stars are close enough for mass transfer to occur from the main-sequence star to the white dwarf (Warner. 1995). The hydrogen-rich material forms an accretion disk which is can be observed by its UV flux and Balmer (e.g. $\mathrm{H} \alpha, \mathrm{H} \beta$ ) emission lines. The accretion disk is also a source of very soft X-ray radiation (Benacquista \& Downing, 2013). CVs in GCs are known to be hard to detect by photometry or X-rays because of their low brightness and crowding. UV observations of GCs with HST resulted in the detection of several CV candidates, but spectroscopy is the gold standard of detecting and confirming CV in GCs (Knigge, 2012). With MUSE data, we were able to increase the number of spectroscopically confirmed CVs in GCs from ten to 17, including two newly discovered systems (Göttgens et al. 2019b. see Chapter 3).

CVs can experience transient events when the accretion disk becomes thermally unstable and its luminosity abruptly increases (dwarf novae, see Hameury 2020 for problems with the disk instability model), or if the accreted material on the white dwarf surface undergoes fusion (classical or recurrent nova). During a classical and recurrent nova, the hydrogen-rich material is accelerated away from the white dwarf and deposited into the interstellar medium. In observations, the ejected material can be seen as a nova shell surrounding the CV (e.g. Shara et al. 2007: Sahman et al., 2015, 2018).

While many classical and recurrent novae have been observed in the Milky Way, only two have been observed in GCs: T Sco in NGC 6093 (M80) observed in 1860 (Sawyer. 1938) and a nova in NGC 6402 (M14) observed in 1938 (Hogg \& Wehlau, 1964).

Black holes (BHs) are known to exist in two different types: stellar-mass BHs with masses between several solar masses up to several tens of solar masses and supermassive BHs with millions or even billions of solar masses. In between these types, hypothetical intermediate-mass black holes (IMBH) could exist (Greene et al. 2020). They are discussed in Section 1.4

Only stellar-mass BHs and IMBHs are of interest for this work since they can (potentially) be found in GCs as supermassive BHs are only found in the centres of massive galaxies. Stellar-mass BHs are the remnants of massive stars that form after the star explodes as a supernova. If the $\mathrm{BH}$ is part of a close binary system with a non-compact companion and mass transfer from the 
companion to the $\mathrm{BH}$ occurs, an accretion disk will form around the $\mathrm{BH}$ that emits $\mathrm{X}$-rays and radio radiation. The first BHs in GCs were detected due to this radiation (Strader et al., 2012, Chomiuk et al., 2013). Later, not only BHs in a mass-transferring binary system were found so far, but also BHs in detached binary systems (Giesers et al. 2018, 2019). In these cases, the radial velocity variation of the visible companion star was used to infer the (minimum) mass of the invisible object, which is too massive to be a white dwarf or a neutron star.

\subsubsection{Multiple populations}

Although the phenomenon of multiple populations in GCs is not directly related to the main topic of this thesis, their discovery changed the perceived astrophysical complexity of GCs since GCs used to be seen as simple stellar populations and the origin of multiple populations remains unsolved (see Bastian \& Lardo, 2018, for a recent review).

High-precision near-UV photometry has shown that the giant branches of all observed MW GCs are split into several distinct branches (e.g. Carretta et al. 2009: Piotto et al. 2015). The number of distinct populations varies from cluster to cluster and also the relative number of stars in each population changes. High-resolution spectroscopy has shown that the colour differences correspond to differences in the abundance of light elements $(\mathrm{C}, \mathrm{N}, \mathrm{O}, \mathrm{Na}, \mathrm{Al}, \mathrm{He}, \mathrm{Mg})$ between each population. There is no difference in the iron abundance or the age between populations of the same GC. Several typical (anti-)correlations between pairs of element abundances have been identified. For example, nitrogen and sodium are correlated while oxygen and nitrogen are anti-correlated. The fraction of stars in the enriched populations (those with higher N, Na and Al abundances) increases with increasing cluster mass, and also the magnitude in the element abundance difference is higher in more massive clusters (see Section 2.5.1 in Bastian \& Lardo, 2018.

These multiple populations are also observed in extra-galactic GC systems but not in massive clusters younger than 2 Gyr. The fact that the approx. $2 \mathrm{Gyr}$ old massive cluster NGC 1978 contains multiple populations illustrates that the formation of multiple populations is not limited to high-redshift environments (Martocchia et al. 2018a). The origin of multiple populations is not yet known. Many different models exist, but so far no model can describe all observed properties of multiple populations (Bastian \& Lardo, 2018). In particular, observations of young massive clusters, the young analogues of ancient GCs observable today, show that they did not undergo multiple epochs of star formation necessary for several theoretical models of multiple populations in 
GCs, and also show that they are free of gas which could enrich any subsequent population (see Section 5.6 in Bastian \& Lardo, 2018, and references therein). For example, photometric studies of stars in two young GCs of the LMC/SMC with multiple populations show that there is no age difference between the populations within each cluster (Martocchia et al., 2018b).

When using data from the MUSE GC survey, combining the observed spectra for a given star can significantly increase the signal-to-noise. This is especially useful when the spectra of all red giants belonging to the same population are combined into a single spectrum that is representative of the population. By comparing the combined spectra of different populations with each other, the element abundance differences can be detected even at the spectral resolution of MUSE (Latour et al. 2019: Saracino et al. 2020: Martocchia et al., 2020).

The large-scale movement of stars belonging to different populations can be different as shown by e.g. Kamann et al.(2020a) using MUSE data of NGC 6093. This cluster has three populations that differ in their nitrogen abundance and the kinematic analysis implies that the population with the highest $\mathrm{Na}$ abundance has a significantly higher angular momentum than the other two while all three populations have the same rotation axis. Initial differences in the rotation of different populations are expected to be still present today, according to $\mathrm{N}$-body models (Hénault-Brunet et al. 2015).

\subsection{Modelling globular clusters}

\subsubsection{Observing the kinematics of globular clusters}

The kinematics of any GC can be split into a large-scale collective motion of the whole cluster around the centre of its host galaxy and the chaotic movements of stars inside the GC. While the collective motion depends on the galactic potential, the individual stellar velocities depend on the gravitational potential of the cluster. Thus, by measuring stellar velocities, we can infer quantities that depend on the gravitational potential such as the mass-to-light profile and the mass of a potential IMBH.

The first systematic observations of a GC to determine the stellar radial velocities were carried out by Wilson \& Coffeen (1954) in M92 and Feast \& Thackeray (1960) in 47 Tuc. These studies used the spectra of 15 and 32 stars, respectively, with distances to the GC centre in the order of arcminutes. The uncertainties on their velocity measurements were too high for a useful estimate of the overall velocity dispersion. Later, Gunn \& Griffin (1979) observed M3 with the intent to measure many individual radial velocities of stars at different radial distances to the GC centre with a high accuracy. Their catalogue con- 
tains individual radial velocities of 111 stars with reported uncertainties about $1 \mathrm{~km} / \mathrm{s}$. The radial separation of these stars is from a few arcminutes down to a few arcseconds. After fitting analytical models to their radially binned velocities, Gunn \& Griffin (1979) conclude that they do not any need heavy remnants other than white dwarfs to explain their data.

Twenty to forty years later, studies with the same aim as Gunn \& Griffin (1979) use velocities of thousands of stars measured with HST and AO-assisted ground-based telescopes. Purely analytical models have been complemented by semi-analytical and completely numerical ones, including $N$-body simulations with several hundred thousand particles. For example, Gebhardt et al. (2000) carried out AO-assisted observations of M15, yielding 1700 velocities when combined with an earlier data set. Five stars with measured radial velocities are within the central $1 \mathrm{arcsec}$ of the cluster. They use their data to conclude that a central dark mass of about $2500 \mathrm{M}_{\odot}$ could be present in M15. As a reference for the total number of published radial velocities of GC stars, Baumgardt \& Hilker (2018) compile 42,000 radial velocities of about 35,000 stars in 109 GCs and claim that $90 \%$ of all stars have velocities with an uncertainty below $2 \mathrm{~km} / \mathrm{s}$. This work is based on the previous compilation of Baumgardt (2017) and it is extended in Baumgardt et al. (2019a).

Similar to radial velocity measurements with spectroscopy, proper motions of individual stars in the crowded cores of GCs are hard to obtain in comparison to stars in the Galactic field. Long-baseline photometric observations of GCs with HST instruments (WFC3 and ACS) are used to derive proper motion of individuals stars in 22 Galactic GCs in the HST Proper Motion project (нSTPROMO). A very impressive example in this regard is their catalogue of omega Cen which contains proper motions of 480,000 stars (Bellini et al., 2017a). Proper motions of individual GCs measured by Gaia are also available for many clusters, although it is usually limited to the less crowded outer regions of the clusters (e.g. Bianchini et al. 2018: Vasiliev \& Baumgardt, 2021). If proper motions from both нSтPRомо and Gaia are available for a given cluster, they can be combined to create a proper motion catalogue that covers both the outer regions as well as the crowded centre (Cordoni et al. 2020b).

With the increasing availability of proper motion and radial velocity data, simultaneous analyses are used to determine the cluster rotation (Sollima et al. 2019: Cordoni et al. 2020a) and the dark contents of a GC (Vitral \& Mamon 2021, but also see Rui et al.2021 and Kremer et al.2021). Sollima et al.(2019) list several advantages of combining radial velocities with proper motions for the analysis of cluster rotation. In particular, it removes a bias since cluster rotation is easier to detect with proper motions if the rotation axis is perpendicular to the sky plane and harder if the axis is in the sky plane, while the reverse is true 
for radial velocities.

The many individual radial velocities in a GC obtained as part of the MUSE GC survey can be used to learn more about the large-scale kinematics of GCs. Kamann et al. (2018) shows that many GCs in the MUSE sample rotate using 500,000 spectra of 200,000 individual stars. Since then, the number of stars has grown to 290,000 distributed over 33 GCs and the number of radial velocity measurements increased to 1.2 million with a median uncertainty of below $10 \mathrm{~km} / \mathrm{s}$ for most clusters. In Section 2.2, the mean radial velocities of about 10,000 stars in NGC 6093 (M80) are used to fit Jeans models to determine the rotation profile and the mass-to-light ratio profile as well as to check if the cluster contains an IMBH.

The analysis of radial velocity time series derived from MUSE data led to the discovery of many binary systems in NGC 3201, several of them with stellarmass black holes (Giesers et al. 2018, 2019). The same data was combined with population tagging to determine if there is a difference in the binary fraction between populations (Kamann et al. 2020c). The full-spectrum fit was modified to include line broadening which allows the measurement of stellar rotation. Due to the relatively large width of the MUSE line spread function, this method is sensitive to velocities above about $20 \mathrm{~km} / \mathrm{s}$. Kamann et al. (2020b) use the stellar rotation velocities measured in the young GC NGC 1846 of the Large Magellanic Cloud to explain the extended main-sequence turn-off in this cluster.

Once the stellar velocities are measured, the question arises how one can infer general cluster properties from this data.

\subsubsection{Analysing motions in globular clusters}

To derive cluster properties from the observed stellar velocities, one has to choose a suitable model for the whole system. The key ingredient is the unknown distribution function (DF) $f(x, v)$ that describes the phase-space density of stars in a collisionless system in equilibrium, usually a galaxy or a GC. There are several different classes of models available in the literature: models based directly on a distribution function, e.g. the models of Varri \& Bertin (2012) and Limepy models (Gieles \& Zocchi. 2015), models that make use of the Jeans equation for collisionless stellar systems (e.g. JAM, Cappellari 2008 and CJAM, Watkins et al. 2013), orbit-based models that use a library of orbits in a given potential (Schwarzschild, 1979), direct $N$-body codes that calculate the gravitational forces on hundreds of thousands of particles (Aarseth, 1999; Nitadori \& Aarseth, 2012), and finally Monte-Carlo codes that use an average potential along an orbit (Giersz, 1998: Joshi et al. 2000). 
Hénault-Brunet et al. (2019) simulate realistic data and compare parameters obtained with different models (including Jeans models, LIMEPY, $N$-body models). They find that each one has its advantages and disadvantages (see their Table 6) and that multimass models based on a distribution function, Jeans models, and $N$-body models reproduce the expected mass profile more accurately.

In the analysis of mock data, usually simple dynamical models are employed: both de Vita et al. (2017) and Aros et al. (2020) use models based on the spherically symmetric version of the Jeans equation and a constant mass-tolight ratio, and the models of de Vita et al. (2017) assume velocity isotropy. As more complex dynamical models are available and are used to analyse real observed data, including such with variable mass-to-light ratio and axisymmetry, the question is what more realistic analyses of simulated observations of GCs with and without IMBH can tell us about the biases and limits of the analyses of real data.

\subsubsection{Jeans models of globular cluster kinematics}

As mentioned in the preceding section, globular clusters can be modelled using solutions of the Jeans equation. This equation is derived from a continuity equation of probabilities and it assumes that the stars in the system are accelerated by a smooth gravitational field generated by all other stars. This assumption is generally true in GCs since the time a star needs to cross the GC is much smaller than the relaxation time of the GC (Binney \& Tremaine, 2008, p.274).

Instead of following stellar particles around their orbit in a stellar system, such as a galaxy or globular cluster, the Jeans equation for a collisionless system in its steady-state describes the spatial dependency of the distribution function $f(\mathbf{q}, \mathbf{p})$ which is proportional to the probability of finding a given star at position $\mathbf{q}$ with momentum $\mathbf{p}$. In analogy to the continuity equation of fluid mechanics or electrodynamics, the conservation of probability inside a small volume in phase space leads to the following equation (Binney \& Tremaine, 2008, eq.4.4):

$$
\frac{\partial f}{\partial t}+\sum_{i=1}^{6} \frac{\partial}{\partial w_{i}} f \dot{w}_{i}=0
$$

where $\mathbf{w}=(\mathbf{q}, \mathbf{p})$ are the phase-space coordinates. Binney \& Tremaine 2008. eq.4.5) show that the second term in this equation can be re-written as derivatives of the Hamiltonian of the system by using the Hamilton equation. By choosing inertial Cartesian coordinates, assuming a steady state (no dependence of any function on time $t$ ), and taking $\Phi$ as the gravitational potential, one arrives at the Boltzmann equation for collisionless systems in equilibrium (Binney \& Tremaine, 2008, eq.4.11): 


$$
\sum_{i=1}^{3}\left(v_{i} \frac{\partial f}{\partial x_{i}}-\frac{\partial \Phi}{\partial x_{i}} \frac{\partial f}{\partial v_{i}}\right)=0 .
$$

For globular clusters, this equation can be simplified by assuming axisymmetry, i.e. all dependencies on the cylindrical coordinate $\phi$ vanish. Since the distribution function can not be observed directly, equations with moments of the distribution function are more useful. By multiplying Eq. 1.2 by the velocities and integrating over them, the two Jeans equations for axisymmetric systems in the steady state are derived (Binney \& Tremaine, 2008, eq.4222):

$$
\begin{array}{r}
\frac{\partial v \overline{v_{R} v_{z}}}{\partial R}+\frac{\partial v \overline{v_{z}^{2}}}{\partial z}+v \frac{\partial \Phi}{\partial z}=0 \\
\frac{\partial v \overline{v_{R}^{2}}}{\partial R}+\frac{\partial v \overline{v_{R} v_{z}}}{\partial z}+v \frac{\overline{v_{R}^{2}}-\overline{v_{\phi}^{2}}}{R}+v \frac{\partial \Phi}{\partial R}=0 .
\end{array}
$$

In these equations, the notation

$$
v \overline{v_{i} v_{j}}=\int f v_{i} v_{j} \mathrm{~d}^{3} \mathbf{v}
$$

is used, where

$$
v(\mathbf{x})=\int f(\mathbf{x}, \mathbf{p}) \mathrm{d}^{3} \mathbf{p}
$$

is the probability to find a star with any momentum at a given location $\mathbf{x}$. The gravitational potential $\Phi$ is generated by the stars and depends on their masses and locations. It can be derived from the observed stellar positions and brightnesses using a mass-to-light ratio $\Upsilon$. With $v$ and $\Phi$ derived from observations, Eqs. 1.3 and 1.4 contain four unknown functions $\overline{v_{R}^{2}}, \overline{v_{\phi}^{2}}, \overline{v_{z}^{2}}$, and $\overline{v_{R} v_{z}}$. The set of equations can be closed by setting $\overline{v_{R} v_{z}}=0$ and relating $v_{z}$ to $v_{R}$ by requiring $\overline{v_{R}}=b \overline{v_{z}}$. The two Jeans equations 1.3 and 1.4 then simplify to

$$
\begin{aligned}
\frac{\partial v \overline{v_{z}^{2}}}{\partial z} & =-v \frac{\partial \Phi}{\partial z}, \\
v \overline{v_{\phi}^{2}} & =b v \overline{v_{z}^{2}}+R b \frac{\partial v \overline{v_{z}^{2}}}{\partial R}+R v \frac{\partial \Phi}{\partial R} .
\end{aligned}
$$

When the gravitational potential $\Phi$ is known, Eq. 1.7 can be integrated to yield the function $v \overline{v_{z}^{2}}$ which can be inserted into Eq. 1.8 to yield $v \overline{v_{\phi}^{2}}$.

To describe the observed spatial stellar distribution in terms of luminosity and density, it is useful to write it as a sum of $N$ Gaussians in the form of a multi-Gaussian expansion (MGE, Emsellem et al., 1994). In case of an equal axial ratio $(q=1)$, the density is described by Gaussians with an amplitude $M_{j}$ and a standard deviation $\sigma_{j}$ (Cappellari, 2008, eq.15):

$$
\rho(R, z)=\sum_{j=1}^{N} \frac{M_{j}}{\left(\sqrt{2 \pi} \sigma_{j}\right)^{3}} \exp \left[-\frac{1}{2 \sigma_{j}^{2}}\left(R^{2}+z^{2}\right)\right] .
$$


This density can be integrated to yield the gravitational potential which is needed to determine the right-hand sides of Eqs. 1.7 and 1.8. The final expression for the velocity dispersion projected along the line-of-sight combines all three components of the 3D velocity dispersion, the inclination angle $i$ of the system, and the MGE amplitudes and widths and can be found in Cappellari 2008. eq.28). Given that the terms $\overline{v_{i}^{2}}$ are the expected value of $v_{i}^{2}$, they can be written as $\overline{v_{i}^{2}}=\sigma_{i}^{2}+{\overline{v_{i}}}^{2}$, where $\sigma_{i}^{2}$ is the variance of $v_{i}$, i.e. the random scatter in the velocity, and $\overline{v_{i}}$ is the expected value of $v_{i}$, i.e. the mean velocity (Cappellari, 2008, eq.32). As $v=0$ for a non-rotating system, the expression for the mean velocity contains an additional parameter, $\kappa$, which scales with the rotation amplitude (Cappellari, 2008, eq.35). Since the usual description for rotation depends on the second moments, $\overline{v_{i}^{2}}$, the mean velocity depends on the same parameters as the second moments.

Figures 1.5 and 1.6 show the mean line-of-sight velocity and its dispersion calculated with CJAM while varying one parameter per row. The shown parameters are the black hole mass $m_{\mathrm{BH}}$, the central mass-to-light ratio $\Upsilon_{0}$, the x-component of the rotation $\kappa_{x}$, the scaling length of the rotation curve $r_{k}$, and the inclination angle of the system. The initial values of the parameters are chosen to resemble the cluster M80. In particular, we assume a system at a distance of $10 \mathrm{kpc}$. As expected, the mean line-of-sight velocity depends on the rotational parameters and the inclination. If the line-of-sight is parallel to the rotational axis (face-on), no variation in the mean velocity can be observed, and thus the rotational parameters cannot be determined from line-of-sight velocities alone. In a case like this, velocities tangential to the line-of-sight can be used to infer the rotation, if available.

The line-of-sight velocity dispersions (Fig. 1.6) depend mostly on $M_{\mathrm{BH}}$ and $\Upsilon_{0}$ : the central black hole causes a central peak in the dispersion which increases with increasing mass. An increasing $\Upsilon_{0}$ has a similar effect but on a larger spatial scale which is set by the width $\sigma_{0}$ of the innermost MGE component. Increasing $\Upsilon_{0}$ corresponds to a larger number of heavy remnants, e.g. stellar-mass BHs, neutron stars, or white dwarfs. The inclination of the system influences the observability of the dispersion: the line-of-sight velocity dispersion is lower if the system is seen edge-on (the opposite of face-on). This is the reverse of the effect of the inclination on the mean line-of-sight velocities. 


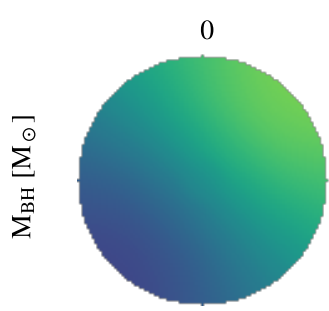

1.5

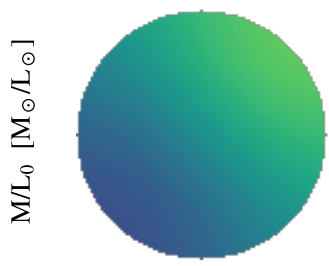

$-0.4$

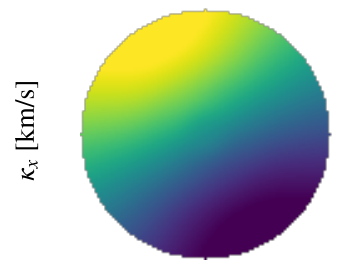

30

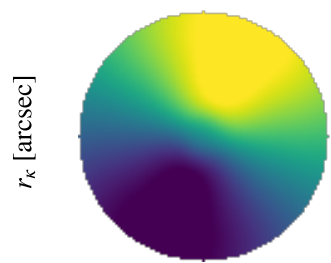

26
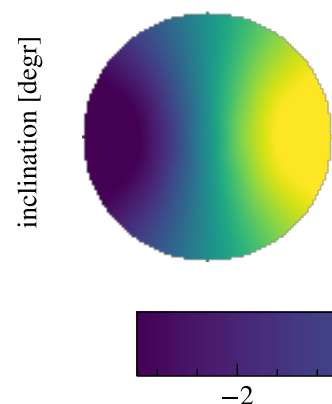

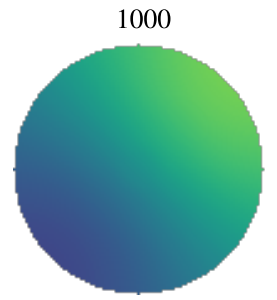

2.0

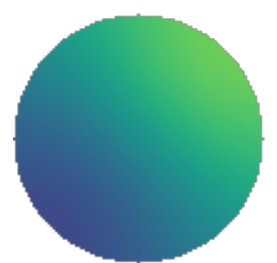

$-0.2$

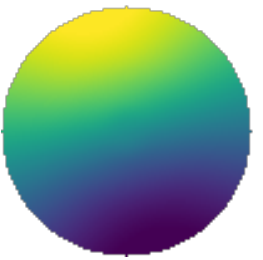

50

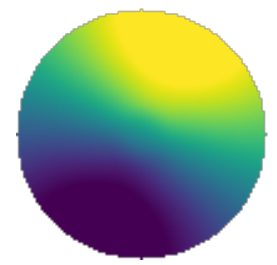

30

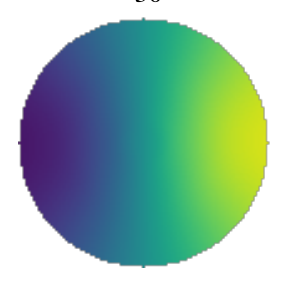

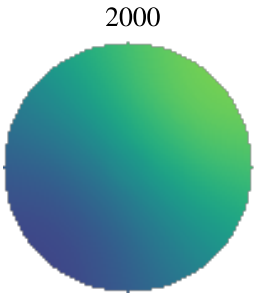

2.5

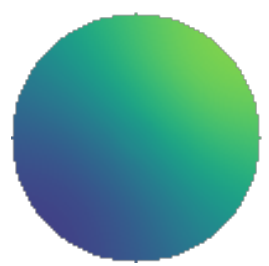

0.2

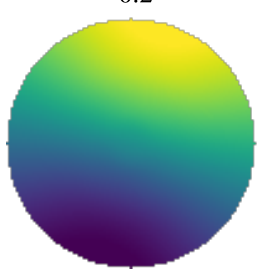

70

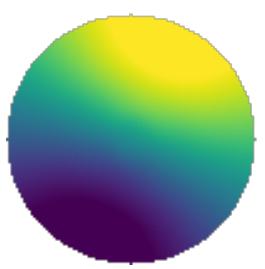

41
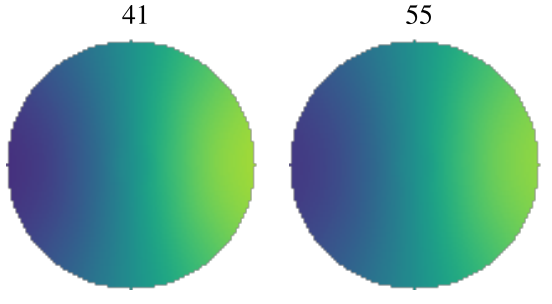

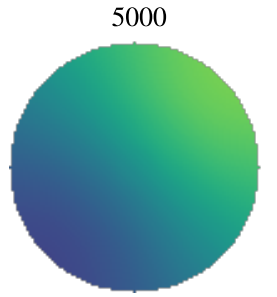

3.0

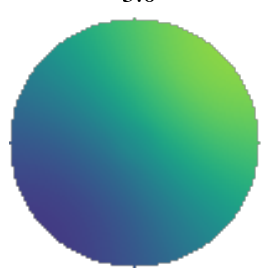

0.4

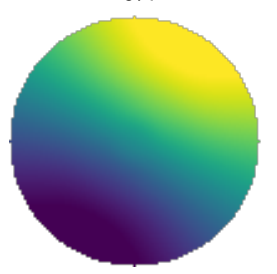

90

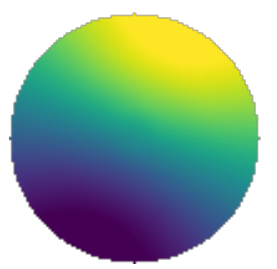

55 . 

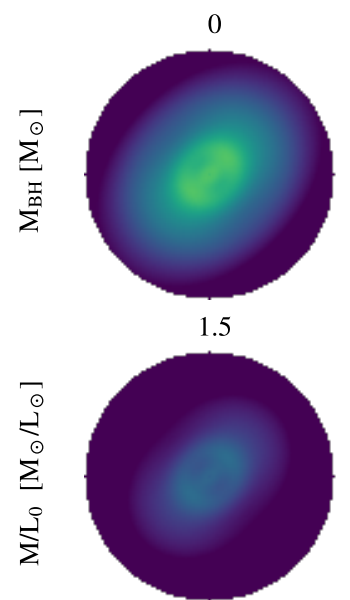

$-0.4$

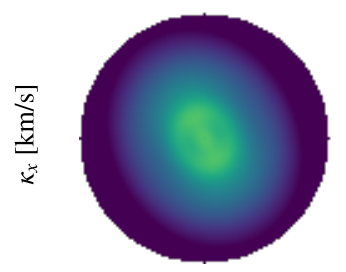

30

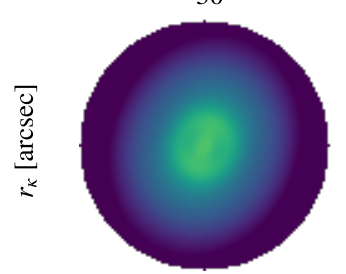

26
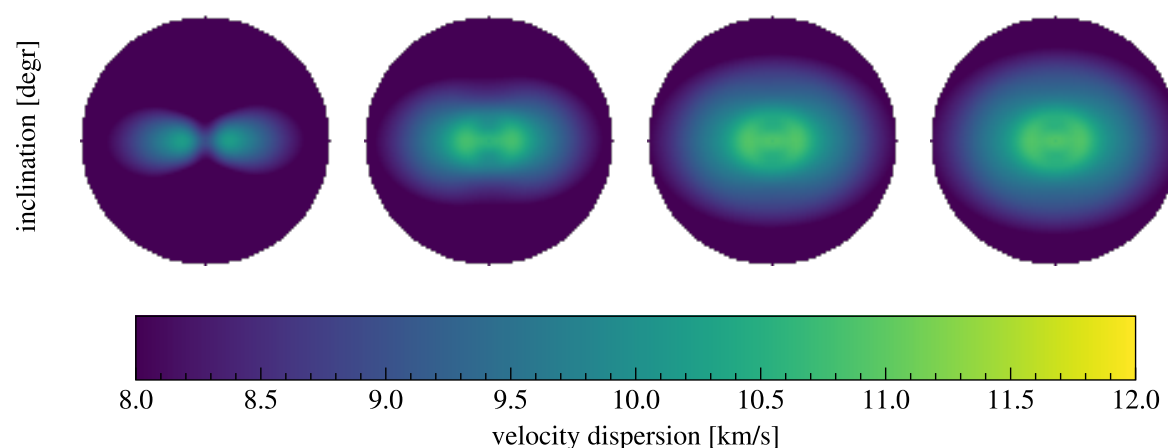

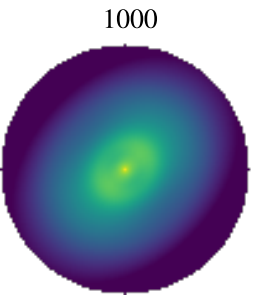

2.0

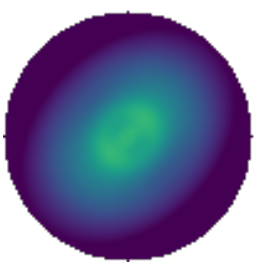

$-0.2$

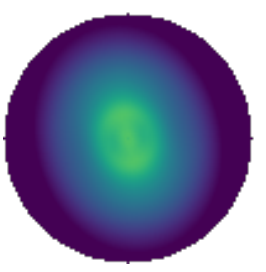

50

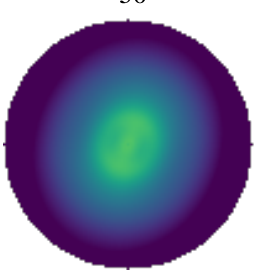

30
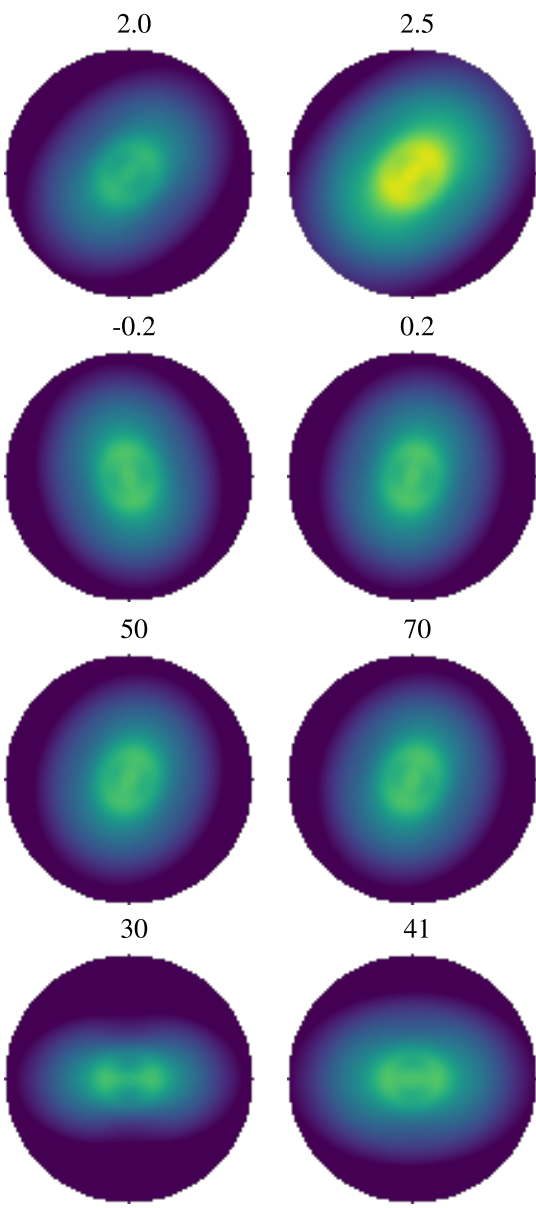

0.2

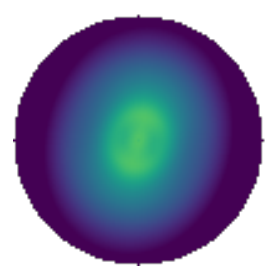

70

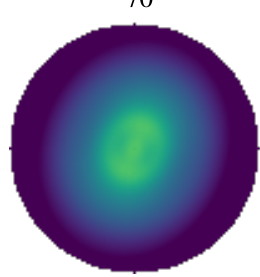

41
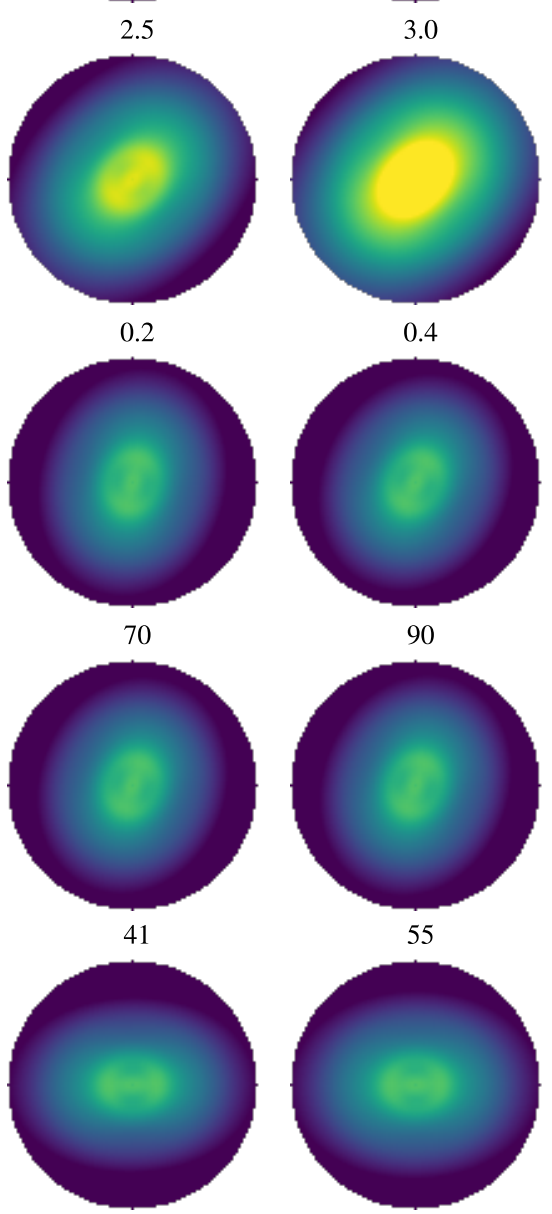

0.4

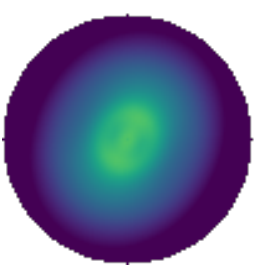

90

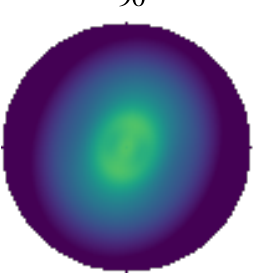

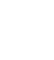

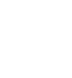

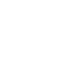




\subsection{Hypothetical intermediate-mass black holes in globular clusters}

As mentioned in Section 1.2.5, intermediate-mass black holes (IMBHs) are a hypothetical class of BHs with a mass of $10^{2}$ to $10^{5} \mathrm{M}_{\odot}$. With these masses, they would fill the perceived gap in the mass distribution of stellar-mass $\mathrm{BH}$ with a few solar masses and supermassive BHs (SMBHs) with masses of more than a million solar masses. SMBHs follow well-known scaling relations, for example between their mass and the velocity dispersion of stars or gas in their environment, i.e. in the centre of galaxies. As the measured velocity dispersion increases, the mass of the SMBH also increases (Gebhardt et al. 2002). Another relation is the one between SMBH mass and the mass of the host galaxy bulge (Magorrian et al. 1998). Assuming that this relation also holds for lower velocity dispersions, the scaling relation implies BHs with several hundred to several thousand solar masses in the centres of GCs. Although this simple extrapolation to lower masses does not have to be true, it motivated a search for IMBHs in GCs both by observations and also by modelling. The existence of IMBHs is also expected from $\mathrm{N}$-body models that show the runaway growth of a star, starting during the core collapse and possibly leading to the formation of an IMBH (Portegies Zwart \& McMillan, 2002). The detection of IMBHs and the shape of their mass distribution could provide clues about the formation mechanism of SMBHs which is currently unknown.

Not all of the about 160 known Galactic GCs are expected to host an IMBH. In particular, core-collapsed GCs are unlikely to contain an IMBH since it would have prevented a core-collapse by quenching mass segregation (Gill et al. 2008). $\mathrm{N}$-body models of GCs with an IMBH show a flat surface brightness profiles, increasing only very close to the GC centre (Baumgardt et al. 2005).

\subsubsection{Detection methods}

Several observation types can be used to detect an IMBH. Radio and X-ray observations of the central regions of a GC would be able to detect emission flux if the hypothetical IMBH is accreting gas from its environment (Maccarone, 2004). Assuming Bondi accretion, a gas accretion efficiency, and a density of intracluster gas which the IMBH could accrete, Tremou et al. (2018) converted their non-detections of radio emission into upper limits on the IMBH mass in 50 Milky Way GCs. Their main result is that there is no evidence for an accreting IMBH with a mass larger than $1000 \mathrm{M}_{\odot}$. This approach works only if there is currently enough intracluster gas available for accretion.

Another approach to find IMBHs in GCs is to detect the gravitational in- 
fluence of the IMBH on the motion of the stars as measured from the radial velocities or their proper motion. If an IMBH is present in the core of a GC, it increases the velocity dispersion of the stars close to it which could be detectable. However, such an increase could also be the result of a larger number of compact sources (neutron stars, stellar-mass black holes) which tend to concentrate in the cluster cores due to mass segregation (e.g. Gieles et al. 2018). Another problem is an anisotropic distribution of velocities which could lead to a seemingly increasing velocity dispersion in the centre if only radial velocities are available for analysis. In this case, the 3D velocity dispersion is unavailable and physically does not increase toward the centre, but the measured dispersion from radial velocities is not representative for the 3D dispersion (Zocchi et al. 2017). As the cluster evolves with time, this anisotropy vanishes as shown by observations and modelling (Watkins et al., 2015: Lützgendorf et al., 2011). As these results show, the velocities of central stars in clusters with an age larger than several times their relaxation time in the cluster core can be treated as isotropic. This is important for the analysis of GCs with only radial velocities since otherwise only analyses with harder to obtain full 3D kinematics would be decisive.

\subsubsection{Extra-galactic IMBH candidates}

Several IMBH discoveries have been made that claim the existence of an IMBH in extra-galactic GCs, in nuclear star clusters of galaxies, in the Galactic disc, and as the source powering ultra- and hyperluminous X-ray sources (e.g. M81-X1 and HLX-1). Nuclear star clusters can be found in the centres of most galaxies and they can co-exist with SMBHs. Compared to GCs, nuclear star clusters are several magnitudes brighter than GCs with a similar mass (see Fig. 2 in Neumayer et al. 2020). There is evidence from the dynamical modelling of gas and other methods that nuclear star clusters of several galaxies contain a black hole with masses in the order of $10^{5} \mathrm{M}_{\odot}$, e.g. NGC 404 (Davis et al. 2020) and NGC 4395 (Brok et al. 2015). In the case of NGC 4395, the BH mass estimate from reverberation mapping is in the order of $10^{4} \mathrm{M}_{\odot}$ (Woo et al. 2019), similar to the IMBH mass expected in GCs. Since the discovery of short X-ray pulsations coming from M81-X2 that can be better explained with an accreting magnetized neutron star instead of an accreting IMBH (Bachetti et al., 2014), it has been accepted that neutron stars and stellar-mass black holes are the accreting objects in many ultra- and hyperluminous X-ray sources. This could be true even for the sources described in a review by Mezcua (2017) as "most well-known off-nuclear IMBH candidate" HLX-1 (see Hameury \& Lasota, 2020) and "the second strongest IMBH among HLXs [hyperluminous X-ray sources]" 
M81-X1 (see Brightman et al. 2020).

Another candidate IMBH is located in G1, which is one of the brightest GCs of the Andromeda galaxy (M31) and has roughly twice the mass of Omega Cen, the largest GC of Milky Way (Meylan et al. 2001). Based on radial velocity measurements, Gebhardt et al. (2002) and Gebhardt et al. (2005) concluded that a IMBH with a mass of $2 \times 10^{4}$ solar masses is needed to explain the observed velocity dispersion profile. Baumgardt et al. (2003) ran $N$-body models and found that the observed profiles can be reproduced by merging two clusters, resulting in a cluster with a relatively high mass-to-light ratio of about four solar masses per solar luminosity but without an IMBH. X-ray observations of G1 showed evidence for accretion coming from the cluster centre (Pooley \& Rappaport, 2006; Kong, 2007), and radio observations of the cluster detected a radio flux that is expected for an accreting IMBH given the observed X-ray flux (Ulvestad et al. 2007). However, simultaneous X-ray and radio observations carried out by Miller-Jones et al. (2012) failed to detect radio emission from G1. They attribute the previously observed radio emission to an outburst of a low-mass X-ray binary, but they can not definitely rule out an IMBH as the $X$-ray source. Although their non-detection does not invalidate the velocity dispersion signal, it shows that even IMBH candidates that are seemingly supported by multiple types of observations (kinematic and electromagnetic) are not secure IMBHs.

\subsubsection{IMBH Candidates in Galactic GCs}

Although Tremou et al. (2018) did not find any evidence of accretion signatures from IMBH in Galactic GCs, several studies based on the interpretation of stellar dynamics have proposed the existence of IMBHs (see Table 3 in Greene et al. 2020). For example, Kizlltan et al. (2017) and Perera et al. (2017) both use the observed timing variation of pulsars in NGC 104 and NGC 6624, respectively, to infer the gravitational potential and thus the contribution of a central IMBH. Although both studies found evidence of such an IMBH, later analyses found dynamical models could describe the observations without an IMBH: In the case of NGC 104, Mann et al. (2019) used proper motions and a model based on the Jeans equations which includes several subcomponents (binaries, low-mass stars, heavy white dwarf, etc.). They find that stellar-mass BHs and binaries concentrated in the centre can explain the observed velocity dispersion well and there is no need for an IMBH in this cluster. Similarly, Hénault-Brunet et al. (2020) model the observed radial velocities and proper motions in NGC 104 without an IMBH to find that they are well described by a central concentration of stellar-mass BHs. In the case of NGC 6624, Gieles et al. (2018) use a similar 
approach as Hénault-Brunet et al. (2020) and also find that their models without IMBH fit well. They explain the observed pulsar timing variations by the influence of passing stars and stellar remnants. Baumgardt et al. (2019b) uses $\mathrm{N}$-body models to find that models of NGC 6624 with IMBH cannot reproduce the observed velocity dispersion or the surface brightness profile, while models without IMBH fit well.

The GC with the most studies on a potential central IMBH has to be Omega Centauri. An IMBH with a mass of 4 to $5 \times 10^{4} \mathrm{M}_{\odot}$ is supported by several studies (Noyola et al., 2008: Jalali et al., 2012: Baumgardt, 2017), while others conclude that there is either no evidence for an IMBH (van der Marel \& Anderson, 2010. Zocchi et al. 2017) or evidence against one (Baumgardt et al. 2019b) in the form of missing high-velocity stars which $\mathrm{N}$-body models with IMBH predict.

In conclusion, there is currently no unambiguous detection of an IMBH in a Galactic GC as papers claiming the discovery of one are quickly followed by studies that explain the data without needing an IMBH.

\subsubsection{Formation scenarios}

There are several formation scenarios of IMBHs (Greene et al. 2020, Section 2.1): direct collapse from gas clouds, accretion onto massive BHs formed by metal-free Population III stars, and gravitational runaway in star clusters. For GCs, the last scenario is the most relevant and it can be further divided into a slow version with a timescale of hundreds of millions of years and a fast version with a timescale of less than several million years. Stellar-mass black holes are one of the proposed ingredients for both the fast and the slower scenario in GCs. While it was for a long time seen as unlikely that more than a single $\mathrm{BH}$ exist in a given GC, the discovery of several stellar-mass BHs has changed this (Strader et al., 2012: Giesers et al., 2018). In the fast formation models, the high stellar density in young GCs leads to the interaction and collision of stars which form very massive stars (VMS) with masses of over 100 solar masses (Portegies Zwart \& McMillan, 2002). In low-metallicity clusters with a high binary fraction of massive stars, the interactions in binaries with massive stars can also lead to the formation of VMS (González et al. 2021). IMBHs can be created through the collision of a VMS with a stellar-mass BH (Rizzuto et al. 2021). The fraction of stellar material that is accreted onto the $\mathrm{BH}$ must be high to form a suitably massive $\mathrm{BH}$ in this step. The IMBH can then continue to moderately grow by further $\mathrm{BH}$ collisions or by accreting material from stars, but it is unclear how long the IMBH stays inside the GC before it gets kicked out. This formation channel is found in N-body simulations (Rizzuto et al., 2021) and Monte-Carlo simulations (Giersz et al., 2015). In Rizzuto et al. (2021), an IMBH 
in a single simulation out of their 80 simulations builds its mass without any VMS, it grows through merging with other $\mathrm{BH}$ instead. The authors compare this merging chain with the gravitational-wave event GW190521 in which two BHs with masses of about 70 and 80 solar masses merged, forming a $\mathrm{BH}$ with a final mass of about 140 solar masses (LIGO Scientific Collaboration and Virgo Collaboration et al., 2020).

\subsection{Aims of his work}

With this work, I aim to explore the wealth of spectral data of globular clusters to discover new unusual astronomical objects. In Section 2.1. I describe the discovery of an emission nebula that was found during an automated search for emission lines in the spectra of GC stars (see Chapter 3 and Section A.1 in the Appendix). This small, unusual nebula has a very low brightness and mass compared to the four known PNe in globular clusters, and its projected distance to the GC centre is very low. This makes it one-of-its-kind and an interesting target for follow-up studies.

In Section 2.2. I try to unambiguously detect the presence of an IMBH in the globular cluster M80. Compared to previous works by others, I use radial velocities of hundreds of stars located in the dense core of M80. This data was obtained with the narrow-field mode of MUSE with a spatial resolution comparable to that of HST ACS. Although several publications on narrow-field mode data from GC exist, this is the first one to use a sophisticated Jeans model to determine the presence of an IMBH. To avoid the same problems as previous publications on the detection of IMBHs in GCs, the paper includes a second analysis of our radial velocities with $\mathrm{N}$-body models. With our flexible Jeans model, we find that the location of the cluster centre is crucial to conclude whether an IMBH exists in this cluster. The methods from this study will be useful in the future, as similar data of other GCs is being analysed.

Finally, Chapter 4 contains conclusions, open questions, and an outlook. 


\section{Chapter 2}

\section{Peer-reviewed journal}

\section{PUBLICATIONS}

\subsection{Discovery of an Old Nova Remnant in the Galac- tic Globular Cluster M22 (Göttgens et al., 2019a)}

The following paper is about the discovery of a emission nebula in M22 which we identified as a nova remnant. However, after receiving suggestions and holding several discussions with external colleagues, we now think this nebula is not a nova remnant but instead connected to GJJC-1 (Gillett et al. 1989), the unusual PN in this GC. This explanation was proposed by George Jacoby and a follow-up paper will be published in the future. While this new interpretation renders obsolete parts of the discussion (Section 4.1), the main discovery and the derived properties of the nebula are still valid.

Most parts of the manuscript were written by me, except for Section 3.1 which was written by P. Weilbacher. P. Weilbacher also extracted the nebular spectra and analysed them with PPXF. All remaining parts of the analysis were carried out by me.

Reproduced with permission from Astronomy \& Astrophysics, ( ESO 


\title{
Discovery of an old nova remnant in the Galactic globular cluster M 22*
}

Fabian Göttgens ${ }^{1}$, Peter M. Weilbacher ${ }^{2}$, Martin M. Roth ${ }^{2}$, Stefan Dreizler ${ }^{1}$, Benjamin Giesers ${ }^{1}$, Tim-Oliver Husser ${ }^{1}$, Sebastian Kamann ${ }^{3}$, Jarle Brinchmann ${ }^{4,5}$, Wolfram Kollatschny ${ }^{1}$, Ana Monreal-Ibero ${ }^{7,8}$, Kasper B. Schmidt ${ }^{2}$, Martin Wendt ${ }^{2,6}$, Lutz Wisotzki ${ }^{2}$, and Roland Bacon ${ }^{9}$

\footnotetext{
${ }^{1}$ Institut für Astrophysik, Georg-August-Universität Göttingen, Friedrich-Hund-Platz 1, 37077 Göttingen, Germany e-mail: fabian.goettgens@uni-goettingen.de

${ }^{2}$ Leibniz-Institut für Astrophysik Potsdam (AIP), An der Sternwarte 16, 14482 Potsdam, Germany

3 Astrophysics Research Institute, Liverpool John Moores University, 146 Brownlow Hill, Liverpool L3 5RF, UK

${ }^{4}$ Instituto de Astrofísica e Ciências do Espaço, Universidade do Porto, CAUP, Rua das Estrelas, 4150-762 Porto, Portugal

5 Leiden Observatory, Leiden University, PO Box 9513, 2300 RA Leiden, The Netherlands

${ }^{6}$ Institut für Physik und Astronomie, Universität Potsdam, Karl-Liebknecht-Str. 24/25, 14476 Golm, Germany

7 Instituto de Astrofísica de Canarias (IAC), 38205 La Laguna, Tenerife, Spain

8 Universidad de La Laguna, Dpto. Astrofísica, 38205 La Laguna, Tenerife, Spain

9 Univ. Lyon, Univ. Lyon1, ENS de Lyon, CNRS, Centre de Recherche Astrophysique de Lyon UMR 5574, 69230 Saint-Genis-Laval, France
}

Received 6 February 2019 / Accepted 8 April 2019

\begin{abstract}
A nova is a cataclysmic event on the surface of a white dwarf in a binary system that increases the overall brightness by several orders of magnitude. Although binary systems with a white dwarf are expected to be overabundant in globular clusters compared with in the Galaxy, only two novae from Galactic globular clusters have been observed. We present the discovery of an emission nebula in the Galactic globular cluster M 22 (NGC 6656) in observations made with the integral-field spectrograph MUSE. We extracted the spectrum of the nebula and used the radial velocity determined from the emission lines to confirm that the nebula is part of NGC 6656. Emission-line ratios were used to determine the electron temperature and density. It is estimated to have a mass of $1-17 \times 10^{-5} M_{\odot}$. This mass and the emission-line ratios indicate that the nebula is a nova remnant. Its position coincides with the reported location of a "guest star", an ancient Chinese term for transients, observed in May 48 BCE. With this discovery, this nova may be one of the oldest confirmed extra-solar events recorded in human history.
\end{abstract}

Key words. globular clusters: individual: NGC 6656 - novae, cataclysmic variables - techniques: imaging spectroscopy

\section{Introduction}

Novae are eruptions on the surface of an accreting white dwarf in a cataclysmic variable $(\mathrm{CV})$ binary system (Iben \& Fujimoto 2008). Hydrogen fusion sets in suddenly when the mass of the accreted hydrogen-rich material on the surface of the white dwarf exceeds a critical value. The energy set free by fusion causes an eruption on the surface and increases the luminosity by several orders of magnitude. The hydrogen-rich matter, possibly mixed with heavier elements of the interior of the white dwarf, is pushed off from the white dwarf with high velocity $\left(>10^{3} \mathrm{~km} \mathrm{~s}^{-1}\right)$ and interacts with the interstellar medium. Although cataclysmic variables are expected to be overabundant in globular clusters (GCs) compared to the Galactic field (Ivanova et al. 2006; Knigge 2012), novae in Galactic globular clusters are very rarely observed. While there are several observations of novae from extra-galactic globular clusters (e.g. Shafter \& Quimby 2007; Henze et al. 2009, 2013; Curtin et al. 2015), there have been only two observations of classical novae (i.e. a CV without multiple observed eruptions) in Galactic globular clusters: T Sco in the core of NGC 6093 (M 80) in 1860

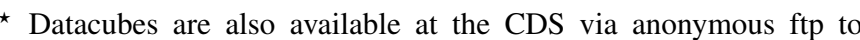
cdsarc.u-strasbg.fr (130.79.128.5) or via http://cdsarc. u-strasbg.fr/viz-bin/qcat?J/A+A/626/A69
}

(Pogson 1860; Sawyer 1938) and a nova in NGC 6402 (M 14) in 1938 (Hogg \& Wehlau 1964).

Supernovae and novae have been known to Chinese, Arabic, Greek, and Babylonian astronomers for thousands of years (Kelley \& Milone 2005). In Chinese records, these new stars are called "guest stars" because they appear, stay for a while and then disappear. The oldest Chinese astronomical inscriptions are 3400 years old and were found on "oracle bones" (Pankenier et al. 2015). Today, we know that supernovae and novae fit the description of guest stars, while comets were usually classified differently (Stephenson \& Green 2009). For example, the supernova that occurred in $1054 \mathrm{CE}$ was described by several Chinese and Arabic sources (Kelley \& Milone 2005), and its remnant is known today as the Crab nebula (M 1). In the case of Nova Scorpii, 1437 CE observed by Korean astronomers, Shara et al. (2017) showed that proper motions can be used to identify the $\mathrm{CV}$ underlying this nova and to determine the age of its remnant independently. Even earlier, a guest star observed by the Chinese in 77 BCE may have been a classical nova outburst of Z Camelopardalis (Shara et al. 2007, 2012; Johansson 2007), although the location of the guest star is very poorly known (Stephenson \& Green 2009).

Emission nebulae created from ejected material allow observers to investigate the respective supernova or nova that 

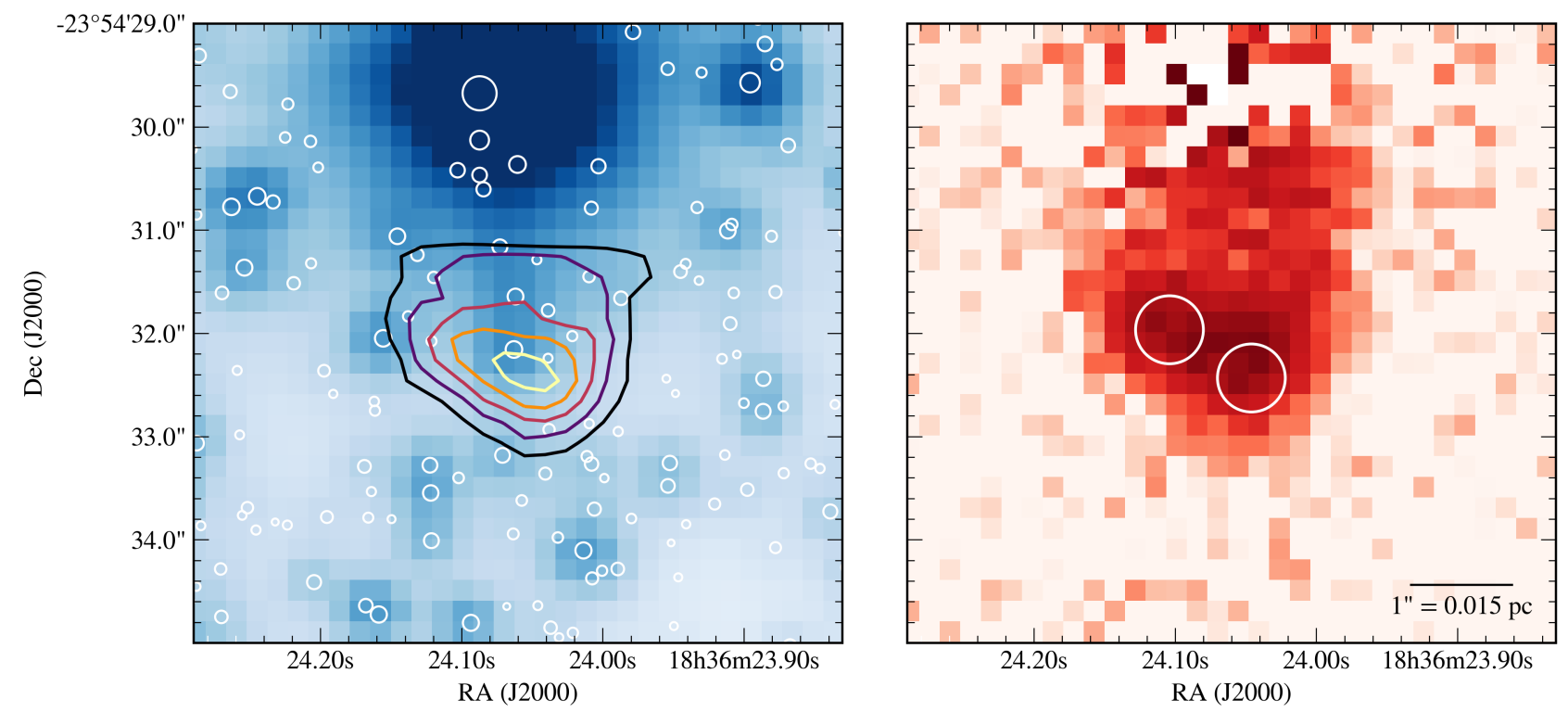

Fig. 1. MUSE whitelight detail image of the region in NGC 6656 containing the nebula created from a single observation (left). Contours give the combined $\left[\mathrm{N}_{\mathrm{II}}\right] \lambda \lambda 6548,6583$ emission flux after subtracting the stellar background (see Sect. 3.1). White circles represent HST sources from the catalogue of Nardiello et al. (2018), the diameter scales with F606W magnitude. The median flux in the four layers containing the [N $\mathrm{II}] \lambda 6583$ emission line is shown after the median flux in three adjacent layers was subtracted (right). The two apertures used to extract the spectrum of the nebula are shown as white circles.

may have happened hundreds or thousands of years ago. Gas inside globular clusters that could be visible as a nebula is also rare (see references in Barmby et al. 2009; Lynch \& Rossano 1990). The only visible occurrences in GCs seem to be planetary nebulae (PNe). While there are thousands of PNe known in the Milky Way disc, only four PNe have been detected in 150 Galactic globular clusters: Ps1 in NGC 7078 (Pease 1928), GJJC-1 in NGC 6656 (Gillett et al. 1989), JaFu-1 in Pal 6, and JaFu-2 in NGC 6441 (both Jacoby et al. 1997).

NGC 6656 (Messier 22) is one of about 150 Galactic globular clusters, its distance to the Sun is $3.2 \mathrm{kpc}$ (Cudworth 1986; Harris 1996, 2010 version). In addition to having a PN, NGC 6656 sticks out from the set of all Galactic globular clusters because it is one of the few for which stellar-mass black holes have been detected. Strader et al. (2012) detected two accreting stellar-mass black holes in this cluster using X-ray and radio observations which they named M22-VLA1 and -VLA2. Using numerical models and observational parameters of Galactic GCs, Askar et al. (2018) predict that NGC 6656 harbours a population of about 30 stellar mass black holes giving rise to its large halflight radius of $1.3 \mathrm{pc}$ (Harris 1996, 2010 version).

\section{MUSE observations and data reduction}

We observed NGC 6656 over a period of seven nights in the period 2015-2017 with MUSE (Bacon et al. 2010), an integralfield spectrograph at the ESO Very Large Telescope (VLT). MUSE has a field of view of $1^{\prime} \times 1^{\prime}$, a spatial sampling of $0^{\prime \prime} .2$, and spectral resolution $R$ between 1800 and 3500 in the spectral range from 4750 to $9350 \AA$. These observations are part of an ongoing survey of 26 Galactic globular clusters (PI: S. Dreizler, Husser et al. 2016; Kamann et al. 2016, 2018; Giesers et al. 2018). For details on the observations and data reduction, we refer to Kamann et al. (2018). Details about observations of NGC 6656 are listed in Table 1, including the image quality measured in the final datacubes after reduction. Each MUSE observation of NGC 6656 has an integration time of ten minutes.
Table 1. MUSE observations of the region containing the nebula.

\begin{tabular}{llll}
\hline \hline Date & ESO prog. ID & Seeing $\left({ }^{\prime \prime}\right)$ & AO \\
\hline 2015-05-12 08:25:22 & 095.D-0629 & 0.50 & No \\
2015-05-12 08:56:52 & 095.D-0629 & 0.74 & No \\
2015-09-11 02:31:19 & 095.D-0629 & 1.16 & No \\
2015-09-12 02:30:09 & 095.D-0629 & 0.74 & No \\
2016-04-08 09:30:13 & 097.D-0295 & 0.84 & No \\
2017-04-23 08:06:35 & 099.D-0019 & 0.86 & No \\
2017-04-23 08:45:43 & 099.D-0019 & 0.76 & No \\
2017-10-23 00:52:38 & 100.D-0161 & 0.80 & Yes \\
2017-10-23 01:09:09 & 100.D-0161 & 0.74 & Yes \\
\hline
\end{tabular}

Notes. The column "Date" corresponds to the mid-observation time, "Seeing" contains the PSF-width measured in the reduced datacubes, and "AO" indicates if the adaptive optics system was used.

\section{A new nebula in NGC 6656}

As part of a systematic search for emission line sources in Galactic globular clusters (Göttgens et al., in prep.), we detected a small emission nebula in NGC 6656 at a distance of about 14" from the cluster centre. The region containing the nebula is shown in Fig. 1 with a MUSE observation collapsed along the spectral direction together with the $\left[\mathrm{N}_{\mathrm{II}}\right] \lambda 6583$ flux after the flux of adjacent layers is subtracted.

\subsection{Flux map and spectral properties}

Flux maps and spectra that are extracted with a simple aperture contain a large amount of stellar flux from sources close to the nebula. To better disentangle stellar background and ionized gas emission, we employed pPXF (Python version 6.7.12, dated 9 July 2018, Cappellari \& Emsellem 2004; Cappellari 2017), the penalized pixel-fitting method that is widely used for fullspectrum fitting with the goal of extracting kinematics of gas and stars and to estimate stellar populations. We chose to describe the spectra with two sets of templates: 


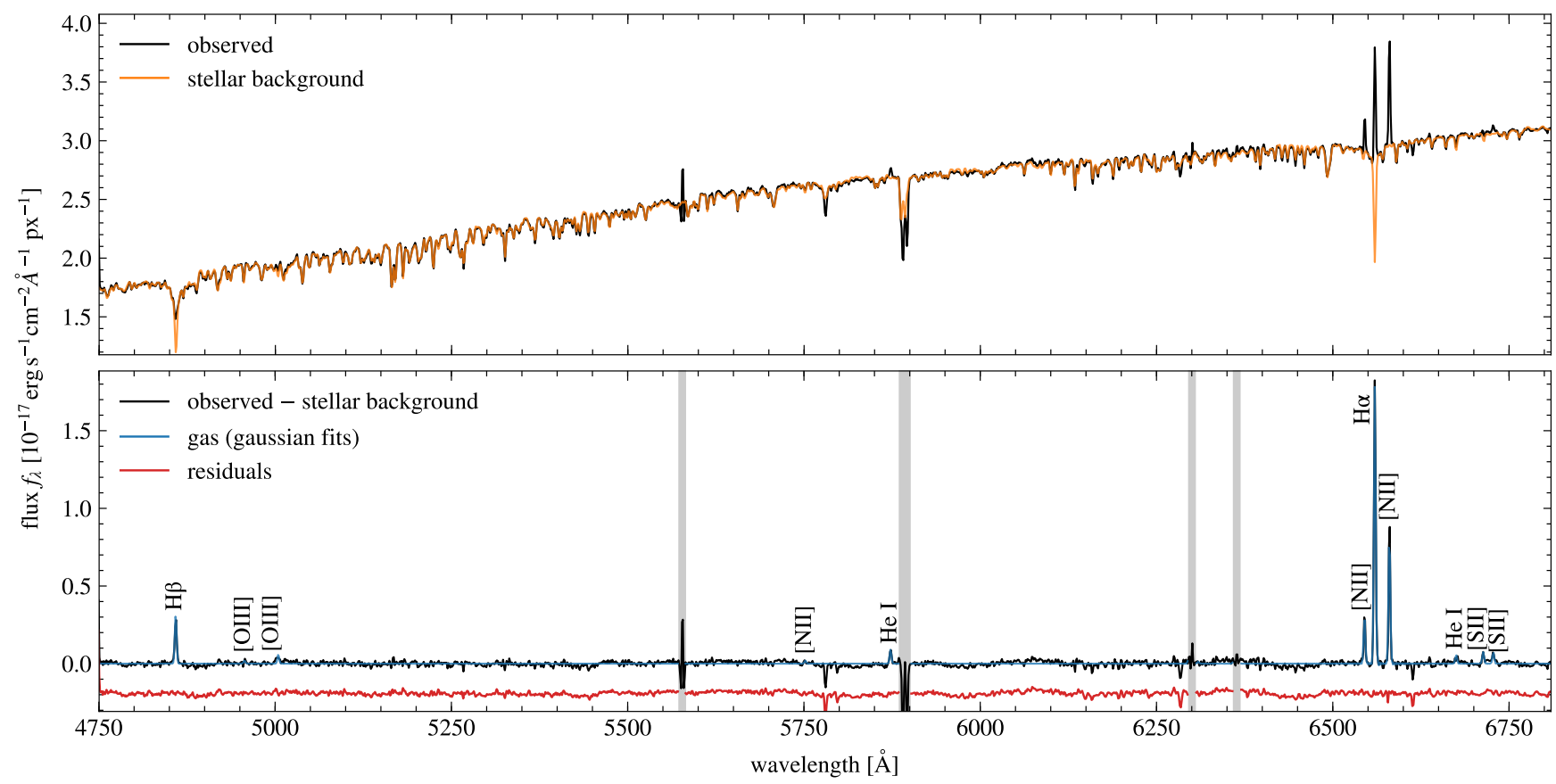

Fig. 2. Average spectrum extracted from two circular aperatures covering the new nebula (top panel, black). This is decomposed into stellar background (top panel, orange) and gas (bottom panel, black and blue). The residuals after fitting Gaussian functions to the emission lines are shown in red (bottom panel). Grey boxes indicate regions dominated by telluric features.

1. The stellar background was modelled using the empirical stellar library MILES (Sánchez-Blázquez et al. 2006; Falcón-Barroso et al. 2011). We took the full library of individual stars that samples the expected range of stars in NGC 6656 well enough. However, we did not preselect the stellar spectra, but let pPXF select the best fit.

2. The gas emission was modelled with a set of Gaussian functions, at the positions of the expected relevant lines (see Table A.1).

pPXF then optimizes the stellar-background fit and also computes emission-line fluxes. We used 100 Monte-Carlo iterations using the fit residuals to estimate errors of the emissionline fluxes. As instrumental width, we took the FWHM of the wavelength-dependent MUSE line spread function as computed by the pipeline, convolved to $1.25 \AA \mathrm{pix}^{-1}$ sampling. As input spectra to pPXF we used two spectra integrated over 0.44 radial apertures (see Fig. 1), placed on the apparent peaks of the $\mathrm{H} \alpha$ emission, as well as all individual spectra in the region around the nebula. We extracted the spectra from a datacube that combined all available non-AO observing epochs ( 21 exposures over seven observations with a total integration time of $70 \mathrm{~min}$ ). Since the AO data has a slightly different wavelength coverage and a broad gap in the region of $\mathrm{NaD}$ and in this case did not actually improve $\mathrm{S} / \mathrm{N}$ or FWHM significantly, we chose not to include them in the combined deep dataset. The contour lines in Fig. 1 give the combined [N II] $] \lambda 6548,6583$ emission flux after subtracting the stellar background. In this map, the nebula appears as an ellipse of $2.5^{\prime \prime} \times 2^{\prime \prime}$, corresponding to $0.04 \mathrm{pc} \times 0.03 \mathrm{pc}$ at the cluster distance of $3.2 \mathrm{kpc}$ (Cudworth 1986). Figure 1 also shows the [N $\left.\mathrm{N}_{\mathrm{II}}\right] \lambda 6583$ spectral layer after subtracting the mean flux of the adjacent layers. While this map does not rely on model assumptions, it contains stronger residuals from the bright star above the nebula. We have made the datacube created from all non-AO observations and the extracted spectrum publicly available ${ }^{1}$.

\footnotetext{
1 https://musegc.uni-goettingen.de/
}

The average spectrum of the two circular apertures is shown in Fig. 2 together with its decomposition into the modelled stellar background and ionised gas. The spectrum clearly contains strong $\mathrm{H} \alpha, \mathrm{H} \beta$ and $\left[\mathrm{N}_{\mathrm{II}}\right] \lambda \lambda 5755,6548,6583$ emission lines, as well as weaker emission lines from [O $\mathrm{III}] \lambda \lambda 4959,5007$, [S II] $\lambda \lambda 6716,6731$, and $\mathrm{He}_{\mathrm{I}} \lambda \lambda 5876,6678$.

Gaussian fits to the emission lines reveal a line-of-sight (LOS) velocity of $-140 \pm 1 \mathrm{~km} \mathrm{~s}^{-1}$. This is consistent with the assumption that the nebula is comoving with NGC 6656 which has a LOS velocity of $-146 \mathrm{~km} \mathrm{~s}^{-1}$ (Harris 1996, 2010 version) and a central LOS velocity dispersion of $9 \mathrm{~km} \mathrm{~s}^{-1}$ (Kamann et al. 2018). The matching LOS velocity and the small apparent separation from the cluster centre of $14^{\prime \prime}$ suggest that the nebula is located inside NGC 6656. We further justify this assumption by comparing the expected nova rates from the cluster and the Galactic field (see Appendix). There are narrow-band H $\alpha$ HST observations taken with WFPC2 of this region but the nebula is not visible in them.

\subsection{Mass of the nebula}

We estimated the mass of the visible nebula using PyNeb (version 1.1.7, dated 18 October 2018, Luridiana et al. 2015) and the equation for the total gas mass given in Corradi et al. (2015) and used in Sahman et al. (2018). Since the total amount of intra-cluster medium in globular clusters is very low (e.g. about $0.3 M_{\odot}$ in the core of NGC 7078, van Loon et al. 2006), we assume that the nebula mass directly corresponds to the mass of the nova ejecta. This is not true for novae in the Galactic field, as the ejecta sweep up interstellar medium which increases the total mass of the nebula and decelerates its expansion (Duerbeck 1987; Shara et al. 2017; Darnley et al. 2019).

We used the measured emission line fluxes (given here in units of $10^{-17} \mathrm{erg} \mathrm{s}^{-1} \mathrm{~cm}^{-2}$ ) including 1- $\sigma$ uncertainties of [N $\left.\mathrm{II}\right]$ $j_{\lambda 5755}=0.06 \pm 0.08$ and $j_{\lambda 6583}=1.8 \pm 0.1$ together with the $[\mathrm{S}$ II] fluxes of $j_{\lambda 6731}=0.15 \pm 0.09$ and $j_{\lambda 6716}=0.13 \pm 0.10$ as input for 


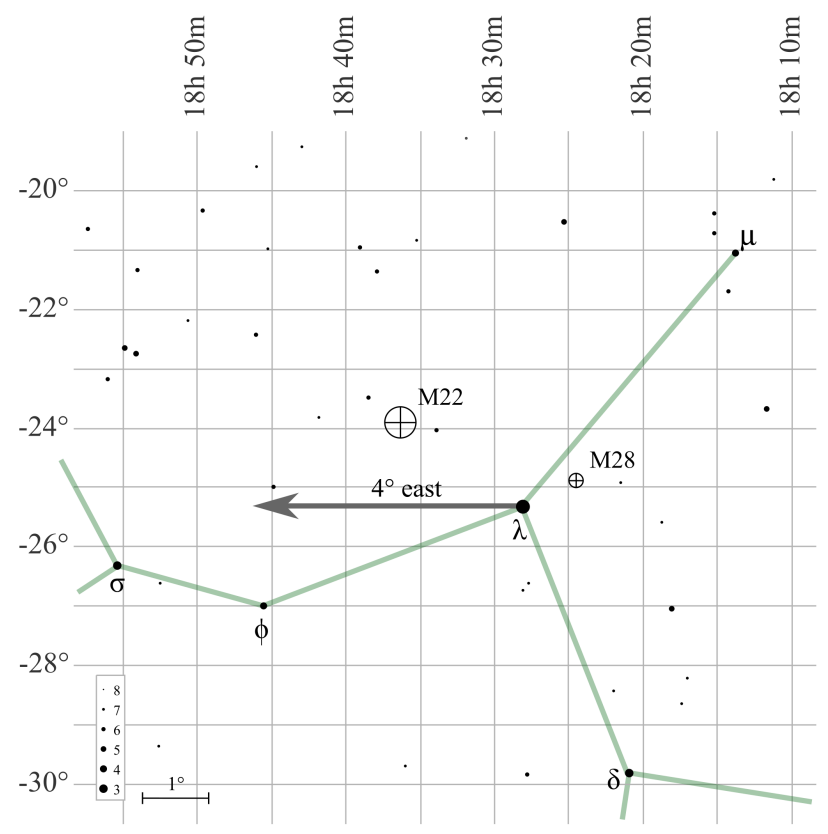

Fig. 3. Chart of the sky close to $\lambda$ Sgr generated as it appeared to a Chinese observer in the May 48 BCE. The grey arrow points to the approximate location of the ancient observation. The globular cluster NGC 6656 (M 22) is located about $2.5^{\circ}$ north-west of this location (about $2.3^{\circ}$ north-east of $\lambda \mathrm{Sgr}$ ). This chart was generated using XEphem (version 3.7.3, Downey 2011).

PyNeb to estimate an electron temperature $T_{\mathrm{e}}=1.8_{-0.8}^{+1.4} \times 10^{4} \mathrm{~K}$ and an electron density $n_{\mathrm{e}}=1.2_{-0.9}^{+3.5} \times 10^{3} \mathrm{~cm}^{-3}$.

To estimate the mass of the nebula, we used the equation given in Corradi et al. (2015) which requires the total dereddened $\mathrm{H} \beta$ flux. We corrected the reddening using PyNeb with the extinction law of Fitzpatrick (1999) and an $E(B-V)=0.34$ (Harris 1996, 2010 version), $R=3.1$, and a distance to the nebula of $3.2 \pm 0.3 \mathrm{kpc}$ (Cudworth 1986). With a total de-reddened $\mathrm{H} \beta$ flux of $(1.9 \pm 0.3) \times 10^{-15} \mathrm{erg} \mathrm{s}^{-1} \mathrm{~cm}^{-2}$, we obtain an estimate for the nebula mass of $1-17 \times 10^{-5} M_{\odot}$. The range takes into account the distance and flux uncertainties as well as the resulting uncertainties in electron temperature and density. This mass estimate is well within both the expected and observed ranges for nova shells (see Table 3 in Yaron et al. 2005) of $10^{-7}-10^{-4} M_{\odot}$ (expected) and $1-30 \times 10^{-5} M_{\odot}$ (observed).

\section{Discussion}

While our mass estimate places the nebula in the range of nova remnants, there are no known novae at this position. This raises the question whether there are other indications of a nova origin.

\subsection{Relation to the guest star $48 B C E$}

Ancient Chinese records in the Book of Han contain a guest star observed in May $48 \mathrm{BCE}$ in the Chinese constellation Nandou (Stephenson \& Green 2009). The name "guest star" was used by Chinese astronomers for what we today call a nova or a supernova. Comets were typically not called guest stars - they had their own category since they could be distinguished by their diffuse appearance and quick apparent motion on the sky. In the Book of Han, the location of the guest star in 48 BCE is given as 4 chi east of the second star in Nandou ( $=\lambda \mathrm{Sgr})$, the separation corresponds roughly to 4 degrees (Stephenson \& Green 2009). Figure 3 illustrates where the stars in the region close to the guest star were located in
May 48 BCE. The globular cluster NGC 6656 is located at $\mathrm{RA}=18^{\mathrm{h}} 36^{\mathrm{m}} 23 \mathrm{~s} .94$, Dec $=-23^{\circ} 54^{\prime} 17^{\prime \prime}$. 1 , about $2.3^{\circ}$ north-east of $\lambda \mathrm{Sgr}$ in the year $48 \mathrm{BCE}$, calculated with astropy ${ }^{2}$ using Gaia DR2 coordinates and proper motions (Gaia Collaboration 2018; Helmi et al. 2018). According to Stephenson \& Green (2009), there is no known supernova remnant within 15 deg of the recorded position. We argue that the guest star observed in $48 \mathrm{BCE}$ was a nova that occurred in NGC 6656 and that it is the remnant of this nova that we have detected with MUSE.

\subsubsection{Measurement errors in the year $48 \mathrm{BCE}$}

The position of the recorded guest star and the observed nova remnant do not coincide exactly. NGC 6656 is rather to the north-east of the reference star instead of east, and the distance is not 4 deg but $2.3 \mathrm{deg}$. However, the uncertain conversion of chi to degrees and measurement errors in the recorded distance and direction have to be taken into account. For example, Shara et al. (2017) use a broad range of conversion factors from 0.44 to $2.8 \mathrm{chi} \mathrm{deg}^{-1}$. As determined by Kiyoshi (1967) and quoted by Ho et al. (1972), the error in stellar positions in observations made approximately 1000 years later is between 0.5 and $1 \mathrm{deg}$. This is the smallest error possible using the techniques available in 48 BCE. The Chinese recording associated with the supernova of 1054, which produced the Crab nebula (M 1), even gives a direction to a reference star that is exactly the opposite of what is observed today (Ho et al. 1972). Given these known inaccuracies of ancient Chinese measurements, we are confident that the guest star position matches the position of NGC 6656.

\subsubsection{Nova and nebula brightness}

Whether a nova from NGC 6656 would be visible to the naked eye depends on the distance to the cluster and the absolute nova brightness. A typical Milky Way nova has an absolute brightness of $-7 \pm 1.4$ mag as determined by Schaefer (2018) using Gaia DR2 parallaxes. Combined with the distance to NGC 6656 (3.2 kpc, corresponding to a distance modulus of $12.38 \mathrm{mag}$, Cudworth 1986), this yields an apparent brightness of $5.38 \pm 1.4 \mathrm{mag}$ for a GC nova. Using this estimate, about $40 \%$ of all novae in NGC 6656 reach an apparent brightness of 5 mag or brighter, which could be seen with the naked eye. About 4-5\% of all novae reach an apparent brightness of at least 3 mag. This shows that the nova that produced the emission nebula in NGC 6656 could have been visible to Chinese observers.

Furthermore, we estimate a brightness from the emission line spectrum without the stellar continuum of $25 \mathrm{mag}$ in the Johnson $V$ band. Using the nova remnant dimming rate of $10 \pm 3 \mathrm{mmag} \mathrm{yr}^{-1}$ (Duerbeck 1992) and the nova brightness distribution above, we obtain an age of $2.0_{-0.5}^{+0.8} \times 10^{3}$ years, which is consistent with the date of the guest star.

\subsection{Alternative interpretations}

There are several types of emission-line objects that have spectra resembling those of nova remnants, for example, planetary nebulae. The mass of a typical Galactic PN is 0.1 to 1 solar masses (Osterbrock 1974), while it can be as low as $10^{-4}-10^{-3} M_{\odot}$ for PNe with a binary central star (Corradi et al. 2015). Since our estimate yields a mass of $1-17 \times 10^{-5} M_{\odot}$ and because of our weak [O III] $] \lambda 5007$ flux, we can exclude a PN as an alternative explanation. In the case of a PN, one would also expect a very hot and bright (post-AGB) central star as the source of the ionisation energy but such a star is not visible in the HST photometry. We

2 Version 3.0.5, www . astropy . org 
can exclude the possibility that the nebula is a supernova remnant because its flux ratio of [S $\mathrm{II}]$ to $\mathrm{H} \alpha$ is lower than the canonical value of 0.4 (Mathewson \& Clarke 1973). Another explanation would be a merger of two stars which increases the overall brightness and thus could look similar to a nova during outburst. The Nova Vulpeculae 1670 seems to have been such a merger (Kamiński et al. 2015), possibly of a white dwarf and a brown dwarf (Eyres et al. 2018). The bipolar nebula identified with this merger was studied extensively, its mass is estimated to be between 0.01 and 0.1 solar masses (Eyres et al. 2018) or even as high as one solar mass (Kamiński et al. 2015). Thus, we can also exclude a stellar merger as a source of the observed nebula. Symbiotic stars, that is, binary systems consisting of interacting red giants and white dwarfs embedded in a nebula fuelled by stellar winds, can have spectra similar to novae. We can exclude a symbiotic star as a mimic because there is no red giant star in the centre of the nebula. We also checked the November 2017 prerelease of the Chandra Source Catalog Release 2.0 (Evans et al. 2010) for X-ray sources close to the nebula that could act as an ionisation source. The only X-ray source in this region is associated with M22-VLA2, one of the two stellar mass black holes in this globular cluster (Strader et al. 2012).

\section{Summary}

We detect a new emission nebula in the globular cluster NGC 6656 using MUSE integral-field observations. After combining exposures from seven observations and modelling the stellar background, we extract a clean spectrum of the nebula. The spectrum has very strong Balmer and [ $\left.\mathrm{N}_{\mathrm{II}}\right]$ emission lines, as well as several weaker emission lines from [O I ], [O III], [S II] and He I. LOS velocity measurements are consistent with the assumption that the nebula is comoving with NGC 6656. We estimate that the mass of the nebula is between 1 and $17 \times 10^{-5} M_{\odot}$, this estimate is well within the typical observed mass range for the ejecta of classical novae and outside the typical values for planetary nebulae or stellar merger remnants, which can have a spectrum similar to that of novae.

Ancient Chinese records of astronomical observations include a guest star, a term used for supernovae and novae, which appeared in $48 \mathrm{BCE}$ within $\sim 2.3^{\circ}$ of the location of NGC 6656 on the sky. The position offset between the recorded guest star and NGC 6656 is within the uncertainty range of ancient observations. The expected absolute visual brightness of novae at the distance of NGC 6656 indicates that a cluster nova would have been visible to the naked eye. We conclude that the nebula detected with MUSE is a nova remnant that was caused by the guest star observed roughly 2000 years ago by Chinese astronomers.

Acknowledgements. We thank the anonymous referee for the constructive report which helped to improve the quality of the work. We also thank Frederic V. Hessman and Francis R. Stephenson for the helpful discussions. FG, SK and SD acknowledge support from the German Research Foundation (DFG) through projects KA 4537/2-1 and DR 281/35-1. SK gratefully acknowledges funding from a European Research Council consolidator grant (ERC-CoG-646928Multi-Pop). PMW, SK, SD and BG also acknowledge support from the German Ministry for Education and Science (BMBF Verbundforschung) through projects MUSE-AO, grants 05A14BAC and 05A14MGA, and MUSE-NFM, grants 05A17MGA and 05A17BAA. JB acknowledges support by FCT/MCTES through national funds by this grant UID/FIS/04434/2019 and through the Investigador FCT Contract No. IF/01654/2014/CP1215/CT0003. AMI acknowledges support from the Spanish MINECO through project AYA2015-68217-P. RB acknowledges support from the ERC advanced grant 339659-MUSICOS. Based on observations made with ESO Telescopes at the La Silla Paranal Observatory under programme IDs 095.D-0629, 097.D-0295, 099.D-0019, and 0100.D-0161. Also based on observations made with the NASA/ESA Hubble Space Telescope, obtained from the data archive at the Space Telescope Science Institute. STScI is operated by the Association of Universities for Research in Astronomy, Inc. under NASA contract NAS 5-26555.

\section{References}

Askar, A., Arca Sedda, M., \& Giersz, M. 2018, MNRAS, 478, 1844

Bacon, R., Accardo, M., Adjali, L., et al. 2010, in Ground-based and Airborne Instrumentation for Astronomy III, International Society for Optics and Photonics, 7735, 773508

Barmby, P., Boyer, M. L., Woodward, C. E., et al. 2009, AJ, 137, 207

Cappellari, M. 2017, MNRAS, 466, 798

Cappellari, M., \& Emsellem, E. 2004, PASP, 116, 138

Corradi, R. L. M., Garcí-Rojas, J., Jones, D., \& Rodríguez-Gil, P. 2015, ApJ, 803, 99

Cudworth, K. M. 1986, AJ, 92, 348

Curtin, C., Shafter, A. W., Pritchet, C. J., et al. 2015, ApJ, 811, 34

Darnley, M. J., Hounsell, R., O’Brien, T. J., et al. 2019, Nature, 565, 460

Downey, E. C. 2011, Astrophysics Source Code Library [recod ascl:1112 . 013]

Duerbeck, H. W. 1987, Astrophys. Space Sci., 131, 461

Duerbeck, H. W. 1992, MNRAS, 258, 629

Evans, I. N., Primini, F. A., Glotfelty, K. J., et al. 2010, ApJS, 189, 37

Eyres, S. P. S., Evans, A., Zijlstra, A., et al. 2018, MNRAS, 481, 4931

Falcón-Barroso, J., Sánchez-Blázquez, P., Vazdekis, A., et al. 2011, A\&A, 532, A95

Fitzpatrick, E. L. 1999, PASP, 111, 63

Gaia Collaboration (Brown, A. G. A., et al.) 2018, A\&A, 616, A1

Giesers, B., Dreizler, S., Husser, T.-O., et al. 2018, MNRAS, 475, L15

Gillett, F. C., Jacoby, G. H., Joyce, R. R., et al. 1989, ApJ, 338, 862

Harris, W. E. 1996, AJ, 112, 1487

Helmi, A., Leeuwen, F. V., McMillan, P. J., et al. 2018, A\&A, 616, A12

Henze, M., Pietsch, W., Haberl, F., et al. 2009, A\&A, 500, 769

Henze, M., Pietsch, W., Haberl, F., et al. 2013, A\&A, 549, A120

Ho, P.-Y., Paar, F. W., \& Parsons, P. W. 1972, Vistas Astron., 13, 1

Hogg, H. S., \& Wehlau, A. 1964, AJ, 69, 141

Husser, T.-O., Kamann, S., Dreizler, S., et al. 2016, A\&A, 588, A148

Iben, Jr., I., \& Fujimoto, M. Y. 2008, in Classical Novae, eds. M. F. Bode, \& A.

Evans (Cambridge: Cambridge University Press), Cambridge Astrophysics Series, 43

Ivanova, N., Heinke, C. O., Rasio, F. A., et al. 2006, MNRAS, 372, 1043

Jacoby, G. H., Morse, J. A., Fullton, L. K., Kwitter, K. B., \& Henry, R. B. C. 1997, AJ, 114, 2611

Johansson, G. H. I. 2007, Nature, 448, 251

Kamann, S., Husser, T.-O., Brinchmann, J., et al. 2016, A\&A, 588, A149

Kamann, S., Husser, T.-O., Dreizler, S., et al. 2018, MNRAS, 473, 5591

Kamiński, T., Menten, K. M., Tylenda, R., et al. 2015, Nature, 520, 322

Kelley, D. H., \& Milone, E. F. 2005, Exploring Ancient Skies: An Encyclopedic Survey of Archaeoastronomy (New York: Springer-Verlag)

Kiyoshi, Y. 1967, Sōgen jidai no kagaku gijutsu shi (Kyoto: Kyoto Daigaku Jimbunkagaku Kenkyusho)

Knigge, C. 2012, Mem. Soc. Astron. It., 83, 549

Luridiana, V., Morisset, C., \& Shaw, R. A. 2015, A\&A, 573, A42

Lynch, D. K., \& Rossano, G. S. 1990, AJ, 100, 719

Marks, M., \& Kroupa, P. 2010, MNRAS, 406, 2000

Mathewson, D. S., \& Clarke, J. N. 1973, ApJ, 180, 725

Nardiello, D., Libralato, M., Piotto, G., et al. 2018, MNRAS, 481, 3382

Osterbrock, D. E. 1974, Astrophysics of Gaseous Nebulae

Pankenier, D. W. 2015, in Handbook of Archaeoastronomy and Ethnoastronomy,

ed. C. L. Ruggles (New York, NY: Springer), 2069

Pease, F. G. 1928, PASP, 40, 342

Pogson, N. 1860, MNRAS, 21, 32

Robin, A. C., Reylé, C., Derrière, S., \& Picaud, S. 2003, A\&A, 409, 523

Sahman, D. I., Dhillon, V. S., Littlefair, S. P., \& Hallinan, G. 2018, MNRAS, 477,4483

Sawyer, H. B. 1938, J. R. Astron. Soc. Canada, 32, 69

Schaefer, B. E. 2018, MNRAS, 481, 3033

Shafter, A. W., \& Quimby, R. M. 2007, ApJ, 671, L121

Shara, M. M., Martin, C. D., Seibert, M., et al. 2007, Nature, 446, 159

Shara, M. M., Mizusawa, T., Zurek, D., et al. 2012, ApJ, 756, 107

Shara, M. M., Iłkiewicz, K., Mikołajewska, J., et al. 2017, Nature, 548, 558

Sánchez-Blázquez, P., Peletier, R. F., Jiménez-Vicente, J., et al. 2006, MNRAS, 371,703

Stephenson, F. R., \& Green, D. A. 2009, J. History of Astron., 40, 31

Strader, J., Chomiuk, L., Maccarone, T. J., Miller-Jones, J. C. A., \& Seth, A. C. 2012, Nature, 490, 71

van Loon, J. T., Stanimirović, S., Evans, A., \& Muller, E. 2006, MNRAS, 365, 1277

Yaron, O., Prialnik, D., Shara, M. M., \& Kovetz, A. 2005, ApJ, 623, 398 


\section{Appendix A: Emission lines used during fitting}

Table A.1. Emission lines that are used to fit Gaussian functions to the observed spectrum.

\begin{tabular}{ll}
\hline \hline Line ID & Air wavelength $(\AA)$ \\
\hline $\mathrm{H} \beta$ & 4861.32 \\
{$[\mathrm{O}$ III] $] 4959$} & 4958.91 \\
{$\left[\mathrm{O}_{\text {III }}\right] 5007$} & 5006.84 \\
$\mathrm{He}_{\text {I }} 5016$ & 5015.68 \\
{$\left[\mathrm{~N}_{\mathrm{I}}\right] 5200$} & 5199.80 \\
{$\left[\mathrm{~N}_{\text {II }}\right] 5755$} & 5754.59 \\
$\mathrm{He}_{\text {I } 5876}$ & 5875.62 \\
{$\left[\mathrm{~S}_{\text {III }}\right] 6312$} & 6312.06 \\
$\mathrm{H} \alpha$ & 6562.79 \\
{$\left[\mathrm{~N}_{\text {II }}\right] 6548$} & 6548.05 \\
{$\left[\mathrm{~N}_{\mathrm{II}}\right] 6583$} & 6583.45 \\
$\mathrm{He}_{\mathrm{I}} 6678$ & 6678.15 \\
{$\left[\mathrm{~S}_{\text {III }}\right] 6716$} & 6716.44 \\
{$\left[\mathrm{~S}_{\text {II }}\right] 6731$} & 6730.82 \\
\hline
\end{tabular}

\section{Appendix B: Relative nova rates}

Throughout the analysis we have assumed that the nebula is located inside NGC 6656 as indictated by its matching LOS velocity and its small separation to the centre of NGC 6656. We further justified this assumption by estimating the relative rate of novae originating from the cluster compared to that of the Milky Way stars in the same region of the sky. The nova rate of a population is the product of its stellar mass and the specific nova rate (i.e. novae rate per solar mass). For the stellar mass, we have to take both foreground and background stars into account because NGC 6656 is located between the solar system and the Galactic centre at a distance of about $3.2 \mathrm{kpc}$. Using a Besançon model ${ }^{3}$ (Robin et al. 2003) for the Milky Way, we estimated a foreground stellar mass in a $0.1^{\circ} \times 0.1^{\circ}$-field centred on NGC 6656 of $10^{3} M_{\odot}\left(3 \times 10^{4} M_{\odot}\right.$ including background $)$, while the cluster has a mass of $2.9 \times 10^{5} M_{\odot}$ (Marks \& Kroupa 2010). If the specific nova rate is the same for the Milky Way and globular clusters, this indicates that there are 300 GC novae per MW nova (or $10 \mathrm{GC}$ novae per MW novae if the total stellar background behind the GC is included). The ratio could even be higher if the specific nova rate is higher in globular clusters compared to the Galactic field, as is the case in M 31 (Henze et al. 2013). In conclusion, a nova remnant with a small apparent separation to the centre of NGC 6656 is more likely to actually originate from the cluster compared to the Galactic fore- and background due to the higher amount of stellar mass in the cluster. 


\subsection{Central kinematics of the Galactic globular clus- ter M80 (Göttgens et al., 2021)}

I wrote most parts of the manuscript, except for Section 5 which was written by $\mathrm{H}$. Baumgardt, who also fit his $\mathrm{N}$-body models to our data. All other analysis steps were carried out by me.

This article has been accepted for publication in Monthly Notices of the Royal Astronomical Society $\odot$ : 2021 The Authors. Published by Oxford University Press on behalf of the Royal Astronomical Society. All rights reserved. 


\title{
Central kinematics of the Galactic globular cluster M80
}

\author{
Fabian Göttgens, ${ }^{1 \star}$ Sebastian Kamann ${ }^{\circledR},{ }^{2 \star}$ Holger Baumgardt ${ }^{\circledR},{ }^{3}$ Stefan Dreizler, ${ }^{1 \star}$ Benjamin Giesers, ${ }^{1}$ \\ Tim-Oliver Husser, ${ }^{1}$ Mark den Brok, ${ }^{4}$ Romain Fétick, ${ }^{5,6}$ Davor Krajnovic ${ }^{4}$ and Peter M. Weilbacher ${ }^{4}$ \\ ${ }^{1}$ Institut für Astrophysik, Georg-August-Universität Göttingen, Friedrich-Hund-Platz 1, D-37077 Göttingen, Germany \\ ${ }^{2}$ Astrophysics Research Institute, Liverpool John Moores University, 146 Brownlow Hill, Liverpool L3 5RF, UK \\ ${ }^{3}$ School of Mathematics and Physics, The University of Queensland, St. Lucia, QLD 4072, Australia \\ ${ }^{4}$ Leibniz-Institut für Astrophysik Potsdam (AIP), An der Sternwarte 16, D-14482 Potsdam, Germany \\ ${ }^{5}$ DOTA, ONERA, Université Paris Saclay, F-92322 Châtillon, France \\ ${ }^{6}$ Aix-Marseille Université, CNRS, CNES, LAM, 13388 Marseille, France
}

Accepted 2021 August 24. Received 2021 August 23; in original form 2021 April 30

\begin{abstract}
We use spectra observed with the integral-field spectrograph Multi Unit Spectroscopic Explorer (MUSE) to reveal the central kinematics of the Galactic globular cluster Messier 80 (M80, NGC 6093). Using observations obtained with the recently commissioned narrow-field mode of MUSE, we are able to analyse 932 stars in the central $7.5 \operatorname{arcsec}$ by 7.5 arcsec of the cluster for which no useful spectra previously existed. Mean radial velocities of individual stars derived from the spectra are compared to predictions from axisymmetric Jeans models, resulting in radial profiles of the velocity dispersion, the rotation amplitude, and the mass-to-light ratio. The new data allow us to search for an intermediate-mass black hole (IMBH) in the centre of the cluster. Our Jeans model finds two similarly probable solutions around different dynamical cluster centres. The first solution has a centre close to the photometric estimates available in the literature and does not need an IMBH to fit the observed kinematics. The second solution contains a location of the cluster centre that is offset by about 2.4 arcsec from the first one and it needs an IMBH mass of $4600_{-1400}^{+1700} \mathrm{M}_{\odot} \cdot N$-body models support the existence of an IMBH in this cluster with a mass of up to $6000 \mathrm{M}_{\odot}$ in this cluster, although models without an IMBH provide a better fit to the observed surface brightness profile. They further indicate that the cluster has lost nearly all stellar-mass black holes. We further discuss the detection of two potential high-velocity stars with radial velocities of $80-90 \mathrm{~km} \mathrm{~s}^{-1}$ relative to the cluster mean.
\end{abstract}

Key words: techniques: imaging spectroscopy -stars: kinematics and dynamics-globular clusters: individual: M80.

\section{INTRODUCTION}

The cores of globular clusters (GCs) are among the regions with the highest density of stars. With up to $10^{5}$ stars per cubic parsec, they contain a multitude of stellar exotica including millisecond pulsars, rejuvenated and heavy stars in form of blue stragglers, cataclysmic variables, accreting and non-accreting stellar-mass black holes as remnants of high-mass stars, and potentially intermediate-mass black holes (IMBHs). IMBHs are a hypothetical class of black holes that would fill the gap between stellar-mass black holes with up to a few tens of solar masses and supermassive black holes in the centres of galaxies with masses ranging from millions to billions of solar masses (Greene, Strader \& Ho 2020). Numerical simulations of GCs predict that they can contain a single IMBH with a mass of several thousand solar masses (Arca Sedda, Askar \& Giersz 2019), formed by merging stellar-mass black holes with massive stars and binaries or by merging massive stars (e.g. Portegies Zwart et al. 2004; Giersz et al. 2015; Rizzuto et al. 2020). An IMBH could be detected through its influence on stellar kinematics as it will increase the velocity dispersion of stars in the centre of a GC. If there is enough gas in

^ E-mail: fabian.goettgens@uni-goettingen.de (FG); S.Kamann@ljmu.ac.uk (SK); dreizler@astro.physik.uni-goettingen.de (SD) the core of a GC, it could be accreted by the IMBH that could cause detectable radio or X-ray emission if the overall accretion efficiency is not too low (Tremou et al. 2018). The deep gravitational potential of an IMBH should cause a change in the period of millisecond pulsars, which could be detectable if all other accelerations are accurately modelled (Kiziltan, Baumgardt \& Loeb 2017; Abbate et al. 2019; Hénault-Brunet et al. 2020). So far, observations have not resulted in a convincing detection of an IMBH in a GC but yielded upper mass limits (see table 3 in Greene et al. 2020). The recent gravitationalwave signal GW190521 points to a heavy BH remnant with a mass of approx. $140 \mathrm{M}_{\odot}$ (Abbott et al. 2020; LIGO Scientific Collaboration and Virgo Collaboration 2020), which is at the low-mass end of IMBHs. It is unclear in which environment this merger occurred, but star clusters provide favourable conditions but star clusters provide favourable conditions for the merging of black holes that are more massive than what is predicted by single-star evolution (Abbott et al. 2020).

A challenge in the detection of IMBHs via stellar kinematics lies in the unknown amount and mass of stellar remnants residing near the cluster centres (e.g. Gieles et al. 2018; Baumgardt et al. 2019; Mann et al. 2019; Zocchi, Gieles \& Hénault-Brunet 2019; HénaultBrunet et al. 2020). However, detailed studies of the central cluster kinematics can help to discriminate between the presence of an IMBH and an overdensity of stellar remnants. In case of $\omega$ Cen 
(NGC 5139), Baumgardt et al. (2019) found using $N$-body models that the proposed $\approx 45000 \mathrm{M}_{\odot} \mathrm{IMBH}$ (Noyola et al. 2010; Jalali et al. 2012; Baumgardt 2017) would produce about 20 stars with a radial velocity above the maximum stellar velocity measured in the centre of this cluster, while models with a number of stellar-mass black holes instead of a central IMBH do not contain these highvelocity stars. IMBHs are statistically expected to have companion stars inside their sphere of influence, which would extend over several tenths of arcseconds to a few arcseconds for most clusters. Although the innermost companion of an IMBH in a 10-Gyr-old GC will typically be a neutron star, a massive $\left(>1 \mathrm{M}_{\odot}\right)$ white dwarf, or a stellar-mass black hole (MacLeod, Trenti \& Ramirez-Ruiz 2016), which are impossible to observe visually, other companions might be observable. Interactions between the IMBH and stars, e.g. a fly-by, could accelerate stars and cause a proper motion or radial velocity much higher than those of stars that did not interact with the IMBH.

M80 (NGC 6093) is an old Milky Way GC with an age of $13.5 \pm 1.0 \mathrm{Gyr}$ (Dotter et al. 2009). It is located in the direction of the Galactic centre at a heliocentric distance of $8.86 \pm 0.55 \mathrm{kpc}$ (Baumgardt \& Hilker 2018). The cluster core radius is $r_{\mathrm{c}} \approx 0.36 \mathrm{pc}=$ 8 arcsec (Harris 1996; Baumgardt \& Hilker 2018). It belongs to the group of dynamically old GCs (Ferraro et al. 2012). While scaling relations derived from GC simulations predict an IMBH with a mass of $(3.63 \pm 0.95) \times 10^{3} \mathrm{M}_{\odot}$ in M80 (Arca Sedda et al. 2019), an integrated-light study did not find evidence for an IMBH (Lützgendorf et al. 2013). Kamann et al. (2020) studied the kinematics of M80 using radial velocities derived from Multi Unit Spectroscopic Explorer (MUSE) data from 2015 to 2017 and the axisymmetric Jeans model from Watkins et al. (2013). This study found that stars belonging to different chemical populations (as discovered by Dalessandro et al. 2018) rotate differently.

In this paper, we build on the study of Kamann et al. (2020) and use new data from the centremost stars in the cluster obtained with the recently commissioned narrow-field mode of MUSE. We combine this with new MUSE wide-field mode data and updated radial velocity estimates (see Sections 2 and 3) to re-analyse the global kinematics of M80 with a Jeans model and calculate rotation profiles, mass-to-light ratio profiles and infer the mass of a hypothetical IMBH (Section 4). Section 5 presents results derived from $N$-body models and the stars with high radial velocities are described in Section 6. We discuss our results in Section 7 and conclude in Section 8.

\section{MUSE OBSERVATIONS}

This study makes use of spectroscopic data taken with the MUSE (Bacon et al. 2010) at the Very Large Telescope as part of the GTO programme 'A stellar census in GCs with MUSE' (PI: S. Dreizler and S. Kamann) which is described in Kamann et al. (2018). MUSE is an optical integral-field spectrograph which is in operation since 2014. Since mid-2019, a new instrument mode (narrow-field mode, NFM) is offered to the community which enables observations with laser tomography adaptive optics (AO) to achieve a higher spatial resolution. NFM observations have a higher spatial sampling of $0.025 \operatorname{arcsec}$ in a smaller field of view of $7.5 \operatorname{arcsec}$ by 7.5 arcsec compared to a sampling of $0.2 \operatorname{arcsec}$ in a 1 arcmin by 1 arcmin field of view in wide-field mode (WFM) observations. While the spatial properties differ, the spectral range in both instrument modes covers 4750-9300 ̊ at a constant sampling of $1.25 \AA$.

We use all available MUSE observations of M80 taken until February 2020. The data analysed here include the 10 WFM observations used in Kamann et al. (2020), 4 new WFM observations, and 2 NFM observations of a single pointing located in the cluster centre. Table A1 lists the five different pointings we observed and the respective instrument mode. The positions of the pointings are shown in Fig. A1.

The main difference of the NFM observations compared to the WFM ones is that we used four instead of three exposures, we did not apply derotator offsets because the natural guide star is off-axis, and the exposure time was $600 \mathrm{~s}$ instead of $200 \mathrm{~s}$ for each exposure. We used the most recent versions (2.6 and 2.8) of the MUSE data reduction pipeline (Weilbacher et al. 2020) to reduce the additional data compared to Kamann et al. (2020).

Fig. 1 compares the image quality of the HST Advanced Camera for Surveys (ACS) Wide Field Channel (WFC) observation using the F606W filter (Sarajedini et al. 2007; Anderson et al. 2008, zoomed on the cluster core), a white-light image created from the MUSE NFM observation, and a MUSE WFM white-light image of the same region derived from the adaptive-optics observation with the best seeing $(0.4$ arcsec). Clearly, the MUSE WFM observation suffers heavily from crowding, while both the ACS and the MUSE NFM image are less affected. As described in detail in Section 3.1 below, we measure an FWHM of around 40 milliarcseconds (40 mas) in the NFM data, i.e. our spatial resolution is higher than that achieved with $A C S$-WFC.

\section{EXTRACTING AND ANALYSING SPECTRA}

While the spectral extraction and spectral analysis for the WFM observations are identical to the procedure explained in Kamann et al. (2020), we modified them for the NFM data. Here, we describe only these changes.

\subsection{Spectral extraction}

We need an initial list of stellar positions and brightnesses at which we extract spectra using PAMPELMUSE (Kamann, Wisotzki \& Roth 2013). The ACS catalogues compiled by Anderson et al. (2008) have been our standard source for this purpose for most clusters in our survey (see Kamann et al. 2018); however, they do not contain all sources visible in the NFM data of M80. ACS images of the cluster core taken in the high-resolution channel are available and we use the catalogue derived by Dalessandro et al. (2018) from these data to improve the source catalogue. After matching stars included in both catalogues using their positions, the final merged catalogue contains 1500 unique stars in the region covered by our NFM observations. However, by visually comparing the combined catalogue with the NFM whitelight image, we estimate that 10-20 percent of all sources visible in the NFM observation still do not have a catalogue entry and thus cannot be extracted. Since these missing stars are all faint, we do not expect that we could obtain useful spectral fits and stellar parameters for them, even if a more complete catalogue was available. We also expect their contribution to the spectra of other stars to be negligible.

PAMPELMUSE reconstructs the shape of the instrumental point spread function (PSF) in a datacube in order to extract spectra. While the PSF shape is the well-known Moffat profile for MUSE WFM observations, it is more complicated in NFM observations. We implemented the PSF model MAOPPY presented in Fétick et al. (2019) in PAMPELMUSE to improve the source extraction. The model is designed for the situation typically faced in adaptive optics, where the PSF consists of a coherent core near the diffraction limit surrounded by a seeing-limited halo, the latter being the result of atmospheric turbulence with spatial frequencies uncorrected for by the deformable mirror. In the case of MAOPPY, the diffraction-limited part of the 

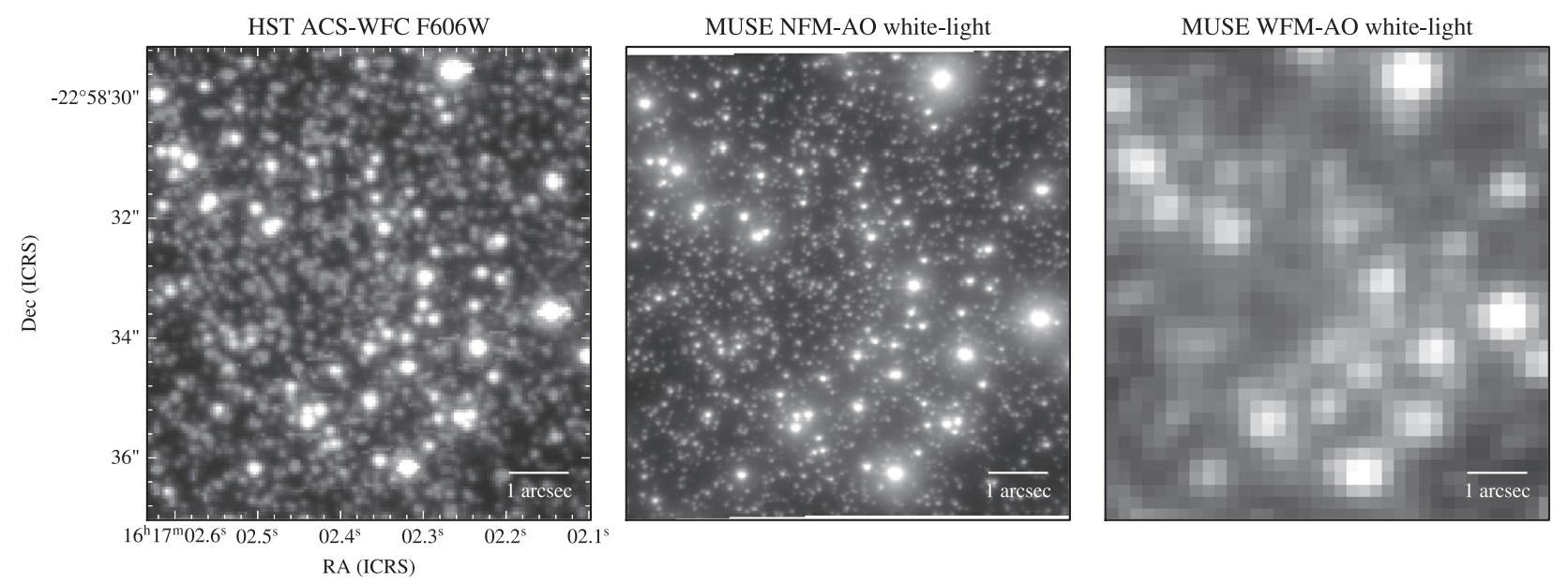

Figure 1. The central 7.5 arcsec by 7.5 arcsec of M80 seen with different instruments. Left: the HST ACS-WFC F606W image (Sarajedini et al. 2007; Anderson et al. 2008), centre: the MUSE NFM white-light image, right: the MUSE WFM-AO white-light image created from the observation with the best seeing $(0.4 \mathrm{arcsec})$. At the cluster distance of $10 \mathrm{kpc}$, a scale of 1 arcsec corresponds to $0.05 \mathrm{pc}$. The most complete catalogue of this region contains about 1500 stars.

PSF is adapted to the instrument in use, whereas the atmospheric residuals in the core are modelled as a Moffat function and the uncorrected spatial frequencies are modelled with the Kolmogorov turbulence model, which includes the Fried parameter $r_{0}$ to scale the turbulence strength. Fig. A2 shows the residuals after PSF and sky subtraction relative to the original data in three different wavelength ranges for the MAOPPY model and a combination of a Moffat profile with a Gaussian core. As the residuals shown in Fig. A2 slightly increase with increasing wavelength, we suspect that the atmospheric diffraction is not completely corrected. We will learn more about the peculiarities of NFM data after reducing more observations. The FWHM of the PSF in our observations decreases from 40 mas in the blue part of the spectrum to 30 mas in the red part. The Strehl ratio is about 5 percent at $650 \mathrm{~nm}$ and increases to a maximum of 10 per cent at $900 \mathrm{~nm}$.

We further noticed that the catalogue positions of a significant fraction of the resolved stars were not accurate enough at the high spatial resolution offered by the NFM. This became evident when we observed significant bipolar fit residuals around the centroids of the affected stars. Recall that by default, PAMPELMUSE predicts the positions of the stars in the MUSE data via a global coordinate transformation from the reference catalogue. The origin of these inaccuracies could be physical (e.g. due to proper motions) or instrumental (e.g. caused by residual errors in the astrometric calibration performed by the data reduction pipeline). In order to account for these offset, a new feature was added to PAMPELMUSE, which allows the user to determine individual offsets $\delta x$ and $\delta y$ for each star relative to the NFM positions predicted by the global coordinate transformation. In the case of M80, typical offsets of 0.4 spatial pixels, corresponding to 10 mas, were applied. For comparison, a star with velocity of $10 \mathrm{~km} \mathrm{~s}^{-1}$ in the plane of the sky at a distance of $10 \mathrm{kpc}$ has moved about 3 mas since the HST observations in 2006 (Anderson et al. 2008). Further details on the improvements implemented in PAMPELMUSE in order to deal with NFM data will be presented in a forthcoming paper.

\subsection{Spectral analysis}

To analyse the extracted spectra, we use our well-tested fitting pipeline described in Husser et al. (2016). Since the adaptive-optics correction works better in the red than in the blue part of the spectrum, the spectral noise is higher in the blue part. To take care of this systematic difference, the full-spectrum fit uses uncertainties derived from the variance extension of the datacube as weights for spectra extracted from NFM observations.

\subsection{Improvements due to NFM observations}

Compared to the previous study of M80 (Kamann et al. 2020), we obtain spectra of more than a thousand new stars in the central 7.5 arcsec by 7.5 arcsec from our NFM observations. Here, we only take into account stars with 'useful' spectra that are spectra with an $\mathrm{S} / \mathrm{N}$ above five, a radial velocity reliability of at least 90 percent (see Section 3.4) and a MagAccuracy of more than 80 percent (see definition in Kamann et al. 2018). We later use radial velocities resulting only from spectra that fulfil these criteria. Fig. 2 shows a colour-magnitude diagram (CMD) of stars inside the NFM region and indicates whether we have observed useful spectra with the WFM, the NFM, or if we did not get a useful spectrum. We analysed the spectra of 185 stars from the WFM observations of the cluster centre, 176 of them have spectra with a mean $\mathrm{S} / \mathrm{N}$ of at least five, and 121 have a mean $\mathrm{S} / \mathrm{N}$ of at least ten. With the NFM observations, we gain 932 stars that had no useful analysis result from previous observations. Of those 932 stars, 891 have spectra with a mean S/N of at least ten. The total number of stars with useful spectra inside the NFM footprint is 1072. This implies an overall completeness of 71 per cent compared to the combined photometric catalogue of Anderson et al. (2008) and Dalessandro et al. (2018) that contains 1501 stars in the region covered by our NFM observations.

\subsection{Filtering data for reliability and binarity}

As described in Kamann et al. (2018), we estimate whether a star is a member of the cluster or a foreground star based on a model of the Galactic stellar population (Robin et al. 2003) in the direction of the cluster. Depending on its mean radial velocity and metallicity, each star is assigned a membership probability.

Regardless of whether a spectrum is extracted from an NFM or WFM observation, its radial velocity derived from the spectral fit is assigned a reliability. This reliability is defined in Giesers et al. (2019, Section 3.2) and it depends on the following properties: the S/N ratio of the respective spectrum, the quality of a cross-correlation with a 


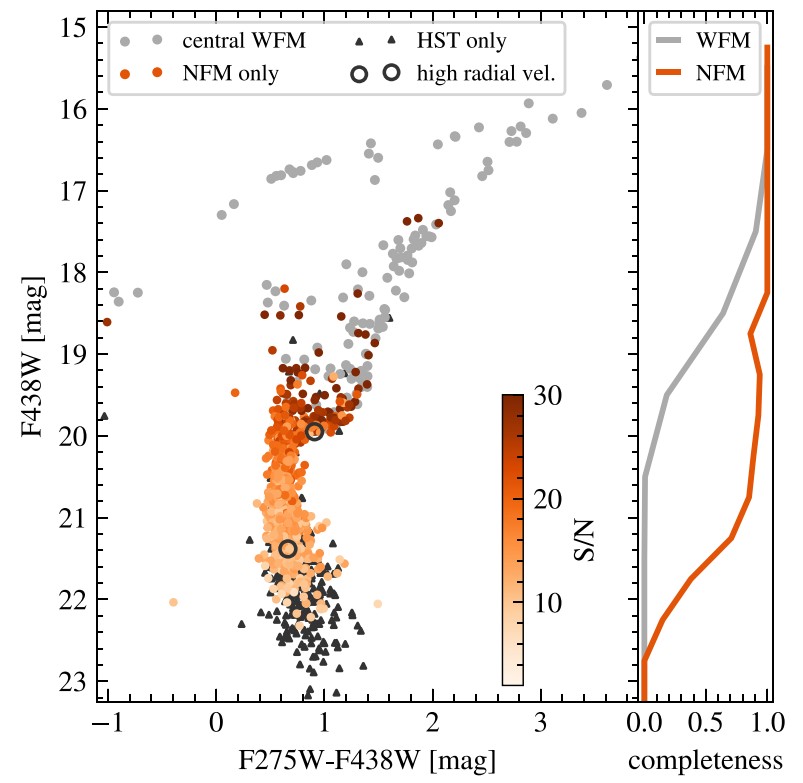

Figure 2. Left-hand panel: Colour-magnitude diagram (CMD) of the central region of M80 using the photometric catalogue of Dalessandro et al. (2018). Grey dots represent stars with spectra extracted from WFM observations while spectra for the red-orange stars could only be obtained from NFM observations. The colour corresponds to the mean $\mathrm{S} / \mathrm{N}$ ratio from our two NFM observations. Grey triangles are HST sources without extracted spectra and grey open circles are the two stars with a high radial velocity (see Section 6). Right-hand panel: our spectral extraction completeness relative to the $H S T$ catalogue for WFM and NFM observations. In both panels, only stars from which we could extract useful radial velocities are taken into account.

suitable template model spectrum, the difference of the radial velocity derived from the cross-correlation and the full-spectrum fit, plausible uncertainties of the radial velocity from the cross-correlation and full-spectrum fit, and agreement between the fitted radial velocity and the mean cluster radial velocity. In this study, we include all radial velocities resulting from a fit with a reliability of more than 90 per cent.

When multiple radial velocities are available for a given star, we use the method described in Giesers et al. (2019) to compute the probability $p$ that they show temporal variability. Since the dynamical model we use is not able to take binary stars into account, we follow Kamann et al. (2020) and only include stars with $p<80$ percent, removing 169 stars from further analysis (about 2 percent of our final sample of stars). We average all reliable radial velocities of the same star weighted by their uncertainties to obtain a mean radial velocity. After filtering, we check the consistency of WMF and NFM observations by computing the mean radial velocity per star separately for WFM and NFM observations. For stars which were observed in both modes, the weighted mean difference of the WFM and NFM velocities is $-0.03 \pm 0.77 \mathrm{~km} \mathrm{~s}^{-1}$. The number of stars in our analysis after filtering is 9720 . In the further analysis, we use the measured radial velocities after subtracting the mean radial velocity of about $9.7 \mathrm{~km} \mathrm{~s}^{-1}$.

This procedure removes two interesting stars very close to the Goldsbury et al. (2010) cluster centre (less than about 1 arcsec) with high radial velocities of $(88 \pm 7)$ and $(101 \pm 4) \mathrm{km} \mathrm{s}^{-1}$ relative to the Solar system barycentre, respectively, and about $10 \mathrm{~km} \mathrm{~s}^{-1}$ less relative to the cluster. It is plausible that both stars are cluster members and not foreground stars because of their low metallicities (both have $[\mathrm{M} / \mathrm{H}] \approx-2$ ) and their positions in the colour-magnitude diagram (on the main-sequence and subgiant branch, see Fig. 2). We discuss these stars in Section 6.

\subsection{External kinematic data}

To increase the coverage of the outer parts of the cluster, we also include the radial velocities of Baumgardt \& Hilker (2018). Stars that appear in both data sets have compatible velocities as shown by Kamann et al. (2020). Before combining these data with ours, we subtract the mean radial velocity from each individual velocity. Baumgardt \& Hilker (2018) provide a probability P_single indicating whether a given star is a single star. We use this information to exclude stars with $\mathrm{P}_{-}$single less than 20 percent and use the remaining 230 stars in our analysis. Their radial distances to the cluster centre are between 20 arcsec and 20 arcmin with a median of 3 arcmin. The total number of stars in our analysis is 9950 . There are no proper motions available for the central regions of M80 because it is not part of the HST proper motion programme (Bellini et al. 2014).

\section{JEANS MODEL}

\subsection{Description}

We use the axisymmetric Jeans model code CJAM of Watkins et al. (2013) which is based on JAM (Cappellari 2008). CJAM was also used in the previous analysis of Kamann et al. (2020). These Jeans models include the effects of anisotropy and rotation to calculate the first and second moments of the velocity distribution at a given point. We compared the model predictions to our data using the same maximum-likelihood approach as in Kamann et al. (2020). The advantage of this approach is that it does not bin the velocities but works with all individual data points. While this increases the computational complexity, it removes the subjective binning step from the analysis.

Since we share the basic model and analyse data of the same GC, we adopt several parameters from the study of Kamann et al. (2020). In particular, we assume an axial ratio of $q=0.9$ for the cluster elongation. We also assume isotropy, i.e. the velocity dispersion along the line of sight and those tangential to it are identical $(\beta=0)$. This assumption is justified by the core relaxation time of $10^{7.78} \mathrm{yr}$ (Harris 1996) that suggests isotropy at the core radius (Watkins et al. 2015, section 5.4).

Compared to the previous study, the model differs in the following aspects:

(i) The coordinates for the cluster centre are no longer fixed to the literature values. Instead, we use two new parameters, $\Delta x$ and $\Delta y$, with Gaussian priors centred on the photometric centre of Goldsbury et al. (2010) to account for uncertainties in the determination of the cluster centre.

(ii) We compute a two-dimensional grid of 960 profiles in the form of multi-Gaussian expansions that cover centre offsets between -3 and +3 arcsec relative to the Goldsbury et al. (2010) photometric centre and use the MGE of the closest grid point to compute the Jeans model during each Markov Chain Monte Carlo (MCMC) step. To construct these profiles, we used the HST photometry of Dalessandro et al. (2018) complemented by the Gaia data of de Boer et al. (2019) as described in Kamann et al. (2020), Section 4.1.

(iii) As suggested by Hogg \& Foreman-Mackey (2018), the rotation field is now parametrized by two vector components, $\kappa_{x}$ and $\kappa_{y}$, instead of its amplitude and an angle. While Kamann et al. (2020) determined the position angle before the main MCMC run, 


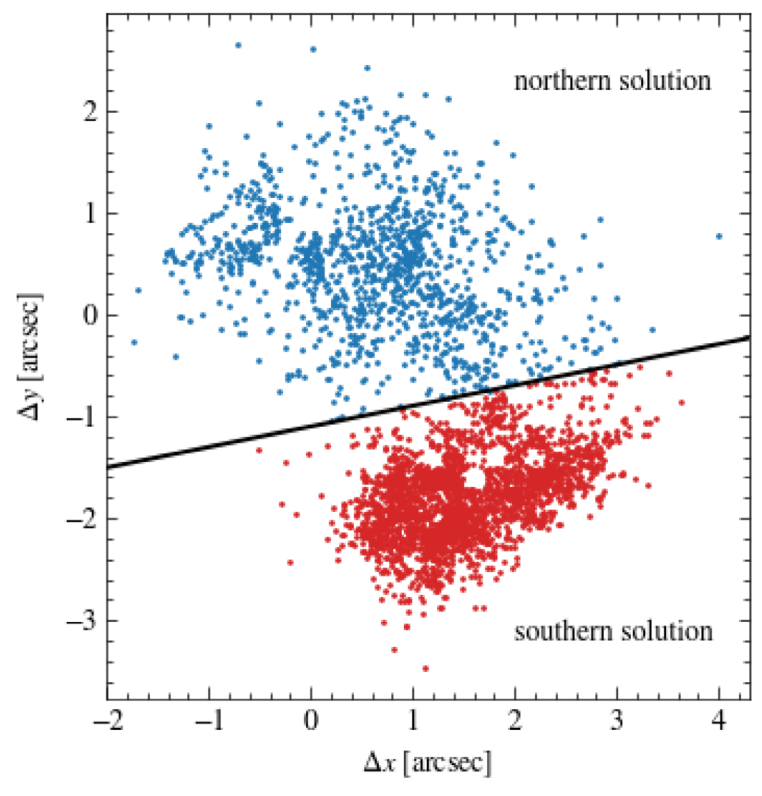

Figure 3. The distribution of centre offsets $\Delta x$ and $\Delta y$ is bimodal. We use the line $Y=0.2 \Delta x-1.1$ arcsec to separate the samples into a northern solution with $\Delta y>Y$ and a southern solution with $\Delta y<Y$.

we vary these parameters together with all remaining ones during the MCMC run. As the Jeans code assumes the semimajor axis to be aligned with the $x$-axis of the coordinate system, we rotate our data by the current position angle estimate prior to calculating a new model.

(iv) We include a potential for an IMBH parametrized by its mass. JAM and CJAM implement this by adding an additional Gaussian component to the MGE. At a mass of zero, the total potential is equal to the potential without a central IMBH.

We take a uniform probability distribution between 0 and $15000 \mathrm{M}_{\odot}$ as a prior for the IMBH mass.

We follow a comment in Cappellari (2008) and fix the standard deviation of the corresponding MGE component to $\sigma_{\mathrm{PSF}} / 3 \approx 25$ mas.

For a given set of parameters, the Jeans code predicts the mean velocity and the velocity dispersion at the location of each star from our kinematic data set. As usual for MCMC approaches, we calculate parameter distributions by maximizing the product of the prior and the likelihood function, which is a Gaussian centred on the difference of measured velocities and the model prediction at that position (Watkins et al. 2013, equation 12). Table A2 lists the eleven parameters and their respective priors. We use the affine invariant ensemble MCMC sampler EMCEE (Foreman-Mackey et al. 2013). We use 192 walkers and a chain length of 2000 after burn-in.

\subsection{Results}

The parameter distributions that we generate with the MCMC sampler (see Fig. A3) are bimodal in the centre offsets $\Delta x$ and $\Delta y$. As shown in Fig. 3, we separate the samples along the line $Y=0.2 \Delta x$ $-1.1 \operatorname{arcsec}$ in the $\Delta x \Delta y$-plane. About one-third of all samples have $\Delta y \geq Y$ and we name them 'northern solution', we name the other two-thirds with $\Delta y<Y$ 'southern solution'. The four circular gaps visible in the southern solution are each centred on a grid point of the MGE grid. The MGE at these locations have one component fewer than the surrounding ones and while they fit the surface brightness profile, they apparently cause a decrease in the likelihood when used as input for the Jeans model to fit the velocities. We present the overall parameter distribution resulting from the MCMC sampling in this section when there is no difference between the northern and the southern solutions and comment on the difference if there is any.

The profile of the mass-to-light ratio $\Upsilon(r)$ has a minimum at $r_{\Upsilon}=1.4_{-0.8}^{+0.9 \prime}$. We find a mean value of $\Upsilon=1.87 \pm 0.15 \mathrm{M}_{\odot} \mathrm{L}_{\odot}{ }^{-1}$ for the cluster which is consistent with the value of $\Upsilon=1.72 \pm$ $0.20 \mathrm{M}_{\odot} \mathrm{L}_{\odot}{ }^{-1}$ determined in Kamann et al. (2020) and also with $\Upsilon=$ $1.93 \pm 0.12 \mathrm{M}_{\odot} \mathrm{L}_{\odot}{ }^{-1}$ from Baumgardt, Sollima \& Hilker (2020). We plot profiles of the mass-to-light ratio $\Upsilon(r)$ using random samples drawn from our chain in Fig. A4. The total cluster mass is $\left(3.0_{-0.3}^{+0.2}\right) \times$ $10^{5} \mathrm{M}_{\odot}$.

For the median intrinsic flattening, we find $\bar{q}=0.86_{-0.05}^{+0.03}$ that is also consistent with $\bar{q}=0.83 \pm 0.06$ found by Kamann et al. (2020). This corresponds to an inclination of $60^{\circ} \pm 15^{\circ}$.

The histograms of all 11 free parameters of the fitted Jeans model and their pairwise correlations are shown in Fig. A3.

\subsubsection{Dispersion and rotation profiles}

To compare if the complex kinematic model actually reproduces our data, we plot the velocity dispersion profiles computed from the parameters in the chain with our data in Fig. 4. This figure also shows a comparison of the observed surface brightness profile and the fitted MGE models.

We binned the data radially and applied a much simpler kinematic model to each bin which determines a constant velocity dispersion and the components $v_{x}$ and $v_{y}$ of the rotational vector. In this model, the predicted radial velocity follows a Gaussian probability distribution with dispersion $\sigma$ and a mean that depends on the model parameters $v_{\text {sys }}, v_{x}$, and $v_{y}$ and on the position angle $\theta$ of a star according to

$v(\theta)=v_{\mathrm{sys}}+\sqrt{v_{x}^{2}+v_{y}^{2}} \sin \left[\theta-\arctan \left(v_{x} / v_{y}\right)\right]$,

where $\theta-\arctan \left(v_{x} / v_{y}\right)$ is the angular distance to the rotation axis. To account for the uncertainty of the cluster centre coordinates, we repeat the binning for 250 potential centres drawn from the MCMC chain. The values from this simple model and the Jeans model agree with each other. Furthermore, Fig. 4 shows the velocity dispersions used in the analysis of M80 by Baumgardt \& Hilker (2018) which also agree with our values. The Jeans model has a central velocity dispersion (at radius of 1 arcsec) of $12.8 \pm 1.3 \mathrm{~km} \mathrm{~s}^{-1}$, which is about $2 \mathrm{~km} \mathrm{~s}^{-1}$ more than the value of $10.5 \pm 0.5 \mathrm{~km} \mathrm{~s}^{-1}$ from Baumgardt \& Hilker (2018). The northern solution has a lower central velocity dispersion of $11.7 \pm 0.6 \mathrm{~km} \mathrm{~s}^{-1}$ and the southern solution has a central velocity dispersion of $13.4 \pm 0.8 \mathrm{~km} \mathrm{~s}^{-1}$. To check if the higher values for the central velocity dispersion of the southern solution are an artefact caused by a few stars with unusual radial velocities, we plotted the radial velocity of individual stars close to the respective centres as a function of the centre distance. We did not find any outliers in these plots. Instead, the overall scatter in the radial velocities is larger around the centre of the southern solution. To further check how reliable the increased velocity dispersion around the southern centre is, we compute the biweight scale of the radial velocities, a robust measure of the standard deviation (see Beers, Flynn \& Gebhardt 1990) for the $n$ nearest neighbours of each star in the NFM field of view $(n=20,40,100)$. This method also shows an increase in the velocity dispersion around the southern centre but not around the northern one.

We find an overall rotation angle of $66^{\circ} \pm 6^{\circ}$, consistent with the angle of $60^{\circ} \pm 3^{\circ}$ found by Kamann et al. (2020). Fig. A5 shows the 

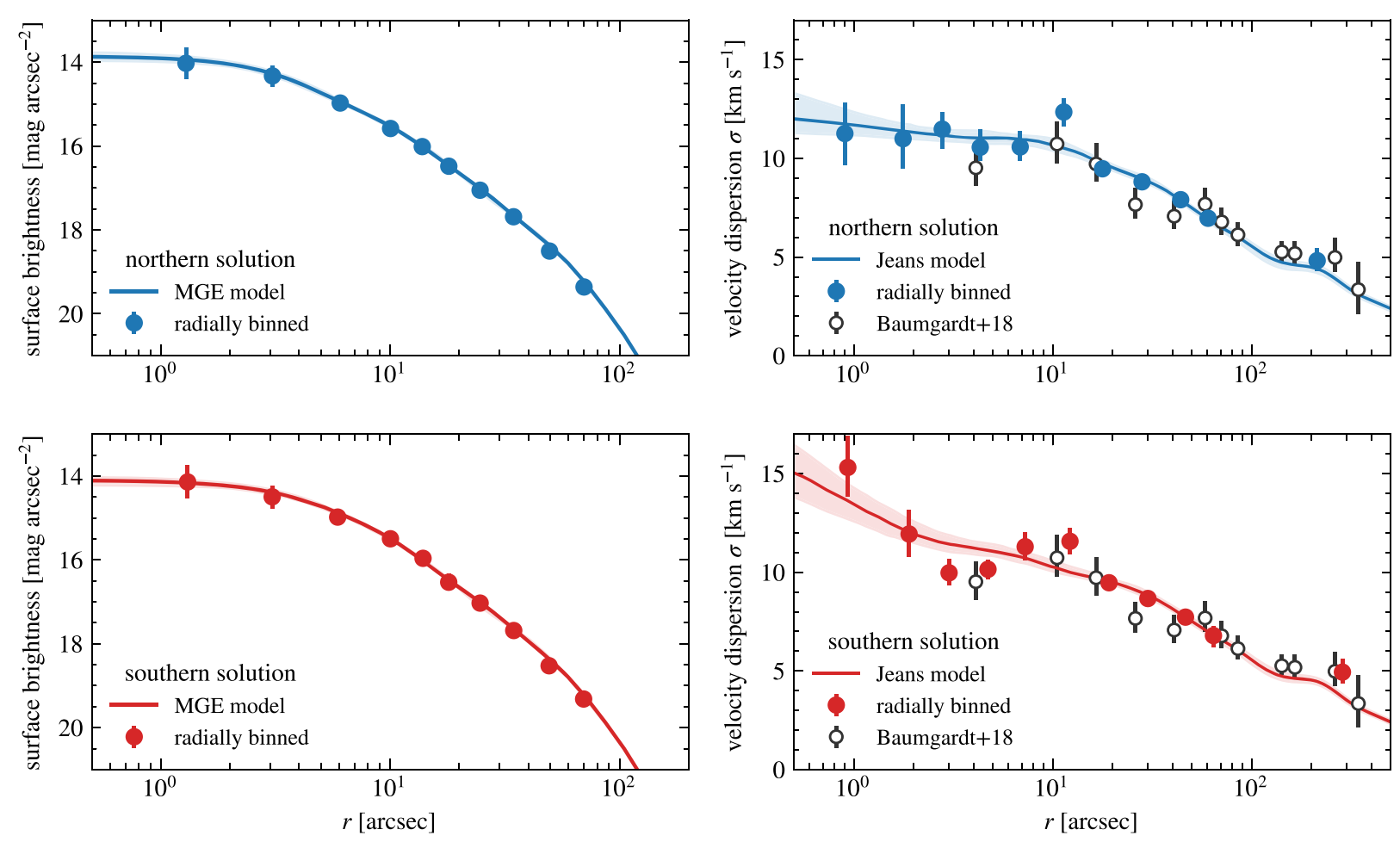

Figure 4. Radial profiles of the surface brightness (left column) and the velocity dispersion (right column). In the left column, filled circles show the profile derived using the photometry presented in Dalessandro et al. (2018), while the solid line shows our best-fitting MGE model. In the right column, filled circles indicate the dispersion obtained directly from the MUSE data, whereas solid lines indicate the predictions from the Jeans models. In all panels, errorbars and shaded areas represent the 14th and 86th percentiles. The top row includes only MCMC samples from the northern solution, the bottom row only from the southern solution. The reference data from Baumgardt \& Hilker (2018) are plotted as published, i.e. without taking into account any centre offset.

radial profile of the rotation amplitude derived from the final MCMC chain. That figure also shows the rotation amplitude computed using the same radial bins and model as for the dispersion plot described above. The radial rotation profile follows the one from Kamann et al. (2020) as expected. The binned data show a very large uncertainty towards the cluster centre because the bin radii are similar to the uncertainty in the centre coordinates.

As a consistency check, we also compute the biweight location of the radial velocities, a robust measure for the mean (Beers et al. 1990), for $n=250$ nearest neighbours of each star in the central 100 arcsec $\times 100$ arcsec. We calculate the rotational parameters from these velocities by treating them as ordinary velocities in our simple model (equation 1). As demonstrated in Fig. A5, the rotational amplitude and angle derived in this way have a similar radial profile as the ones directly computed from the radial velocities.

We had a closer look at whether the core of M80 rotates. While such a central rotation component is expected to be shortlived because of the effects of two-body relaxation, evidence for a decoupled core has for example been reported in the core collapse cluster NGC 7078 (van den Bosch et al. 2006; Usher et al. 2021). The central rotation curve strongly depends on the position of the cluster centre since we use the radial distance to the centre for binning. To account for the uncertainty in the position of the cluster centre, we draw 250 pairs of the centre position offsets $\Delta x$ and $\Delta y$ from our final MCMC chain. Fig. 5 shows the joint distribution of the $x$ - and $y$-component of the rotation velocity for different radial bins. While we find a clear rotation signal of $v_{\text {rot }}=1.9 \pm 0.4 \mathrm{~km} \mathrm{~s}^{-1}$ in the outer parts of the cluster, similar to the value of $1.97 \pm 0.84 \mathrm{~km} \mathrm{~s}^{-1}$ determined by Sollima, Baumgardt \& Hilker (2019, table 1), it is less clear if the cluster core $\left(r_{\text {core }} \approx 8\right.$ arcsec, Harris 1996; Baumgardt $\&$ Hilker 2018) is rotating. In this region, the rotation components follow a broad distribution with a median of $0.9 \mathrm{~km} \mathrm{~s}^{-1}, 90$ per cent of all samples are below $v_{\text {rot }}=1.6 \mathrm{~km} \mathrm{~s}^{-1}$. We note that the central rotation angle is offset from the rotation angle in the outer parts of the cluster by about $150^{\circ} \pm 30^{\circ}$.

\subsubsection{Position of the cluster centre}

The centres of GCs are usually determined using photometric data. There are three recent measurements for the cluster centre of M80. Goldsbury et al. (2010) found RA $=16^{\mathrm{h}} 17^{\mathrm{m}} 2.41$ and Dec. $=$ $-22^{\circ} 58^{\prime} 33^{\prime \prime} .9$ with an uncertainty of 0.2 arcsec, while Dalessandro et al. (2018) found RA $=16^{\mathrm{h}} 17^{\mathrm{m}} 2.481$ and Dec. $=-22^{\circ} 58^{\prime} 34^{\prime \prime} .098$ with an uncertainty of 0.17 arcsec, and Lützgendorf et al. (2013) found $\mathrm{RA}=16^{\mathrm{h}} 17^{\mathrm{m}} 2^{\mathrm{s}} .4$ and Dec. $=-22^{\circ} 58^{\prime} 32^{\prime \prime} .6$ with an uncertainty of 0.7 arcsec. We compare these centres with the offsets in the centre position as sampled by our MCMC chain by transforming the offsets to equatorial coordinates and plotting their density in Fig. 6. The centre of the northern solution is located at

$\mathrm{RA}=16^{\mathrm{h}} 17^{\mathrm{m}} 2^{\mathrm{s}} .36$, Dec. $=-22^{\circ} 58^{\prime} 33^{\prime \prime} .4$,

and the centre of the southern solution is located at

$\mathrm{RA}=16^{\mathrm{h}} 17^{\mathrm{m}} 2^{\mathrm{s}} \cdot 30$, Dec. $=-22^{\circ} 58^{\prime} 35^{\prime \prime} .7$.

As these centres are determined from a dynamical model and to distinguish it from the photometric centres, we call them dynamical centres. The contours around the dynamical centres in Fig. 6 show the uncertainties which are larger than those for the photometric 


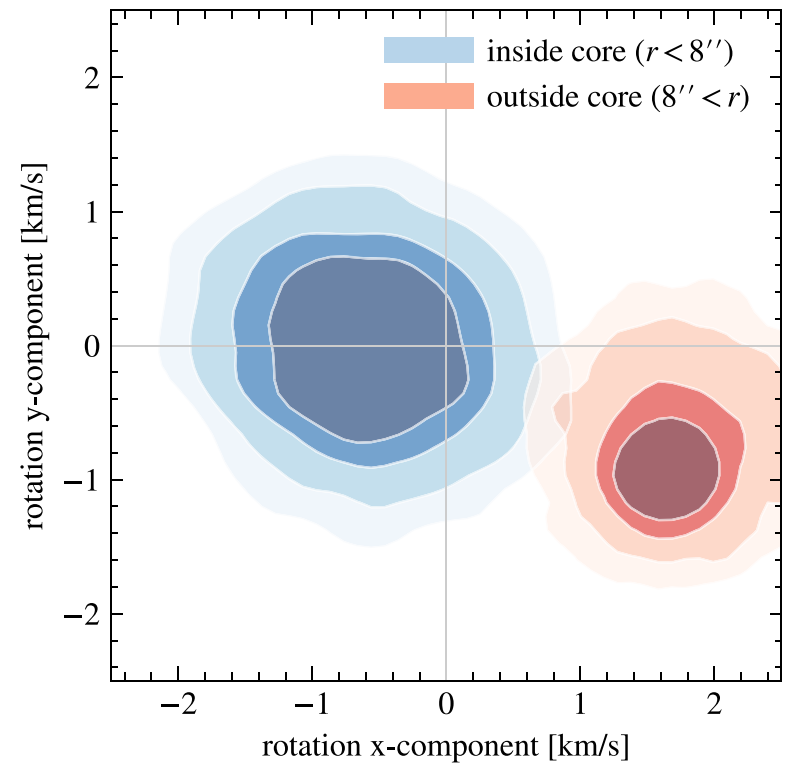

Figure 5. Joint distribution of the estimated $x$ - and $y$-component of the rotation velocity. Each set of contours contains 50, 68, 87, and 95 per cent of all samples (from inner- to outermost) belonging to the core region (blue) or the outer region (red) of M80. The outer region is rotating, but it is not clear if the cluster core $(r<8 \mathrm{arcsec})$ is also rotating.

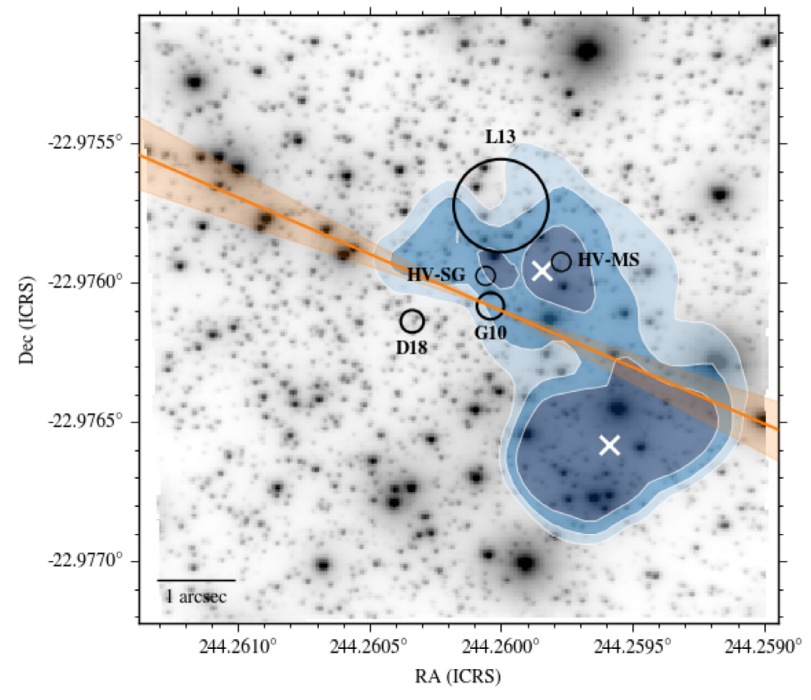

Figure 6. Contour plot of our estimate of the kinematic centre of M80. The blue-shaded regions cumulatively contain 68,87 , and 95 per cent of all samples (from inner- to outermost). The two white crosses are the centres of the northern and the southern solution. G10 denotes the centre found in Goldsbury et al. (2010), L13 is from Lützgendorf et al. (2013), and D18 is the centre of Dalessandro et al. (2018). The smaller circles mark the potential central high-velocity main-sequence (HV-MS) and subgiant (HVSG) stars. The orange line indicates the rotation axis of the whole cluster and its uncertainty computed from the 16th and 84th percentiles, using G10 as the reference centre.

centres. While the centre of the northern solution is close to the known photometric centres, especially to the ones from Goldsbury et al. (2010) and Lützgendorf et al. (2013), the southern centre is located at a distance of about 2.4 arcsec to the south-west of the northern centre.

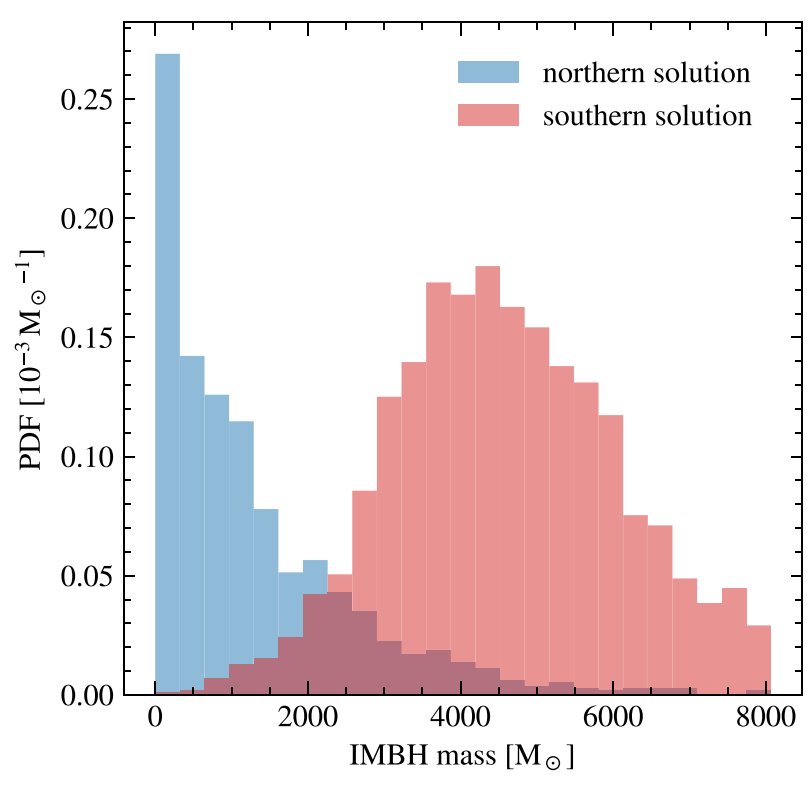

Figure 7. Distribution of the IMBH masses obtained via Jeans modelling around different centres.

\subsubsection{Limits on the IMBH mass}

The posterior distribution of the mass has a peak at masses below $1000 \mathrm{M}_{\odot}$ and another peak at about $5000 \mathrm{M}_{\odot}$. The uncertain position of the centre has a large impact on the shape and moments of the distribution of the IMBH mass, as can be seen from Fig. 7. Here, we compare the IMBH mass distributions of the northern and the southern solutions. The 90 per cent upper limits on the IMBH mass (rounded to the nearest $100 \mathrm{M}_{\odot}$ ) are 3000 and $6800 \mathrm{M}_{\odot}$, respectively. Given a peaked shape of the IMBH mass distributions for the southern centre, we also report a median IMBH mass and an $1 \sigma$ uncertainty of $4600_{-1400}^{+1700} \mathrm{M}_{\odot}$ around this centre.

\section{N-BODY MODELS}

In order to further investigate if the observed velocity dispersion of M80 requires the presence of an $\mathrm{IMBH}$, we fit a grid of $N$ body models against the observed surface brightness and velocity dispersion profiles. In particular, we use the grid of IMBH models presented by Baumgardt (2017) and the grid of models with varying stellar-mass back hole retention fractions presented in Baumgardt et al. (2019). Baumgardt (2017) and Baumgardt et al. (2019) have run a grid of about $3000 \mathrm{~N}$-body simulations of star clusters containing $N=100000$ or $N=200000$ stars using NBODY6 (Aarseth 1999; Nitadori \& Aarseth 2012), varying the initial density profile and half-mass radius, the initial mass function and the mass fraction of an IMBH in the clusters. Fig. 8 compares the best-fitting models without an IMBH and with IMBHs containing 0.5, 1.0, 2.0 and 5.0 per cent of the final cluster mass of $3 \times 10^{5} \mathrm{M}_{\odot}$ with the observed velocity dispersion profile and the observed surface brightness profile for the northern and southern centre solutions (also see Tables A3 and A4). Around the southern centre it can be seen that models with an IMBH lead to somewhat better fits of the velocity dispersion profile, whereas the surface brightness profile is better reproduced by the models without an IMBH. Measuring the error weighted difference between observed and predicted velocity dispersion and dividing by the number of degrees of freedom, we obtain a reduced $\chi_{r}^{2}$ value of 1.54 for the 2 per cent IMBH model from the fit to 

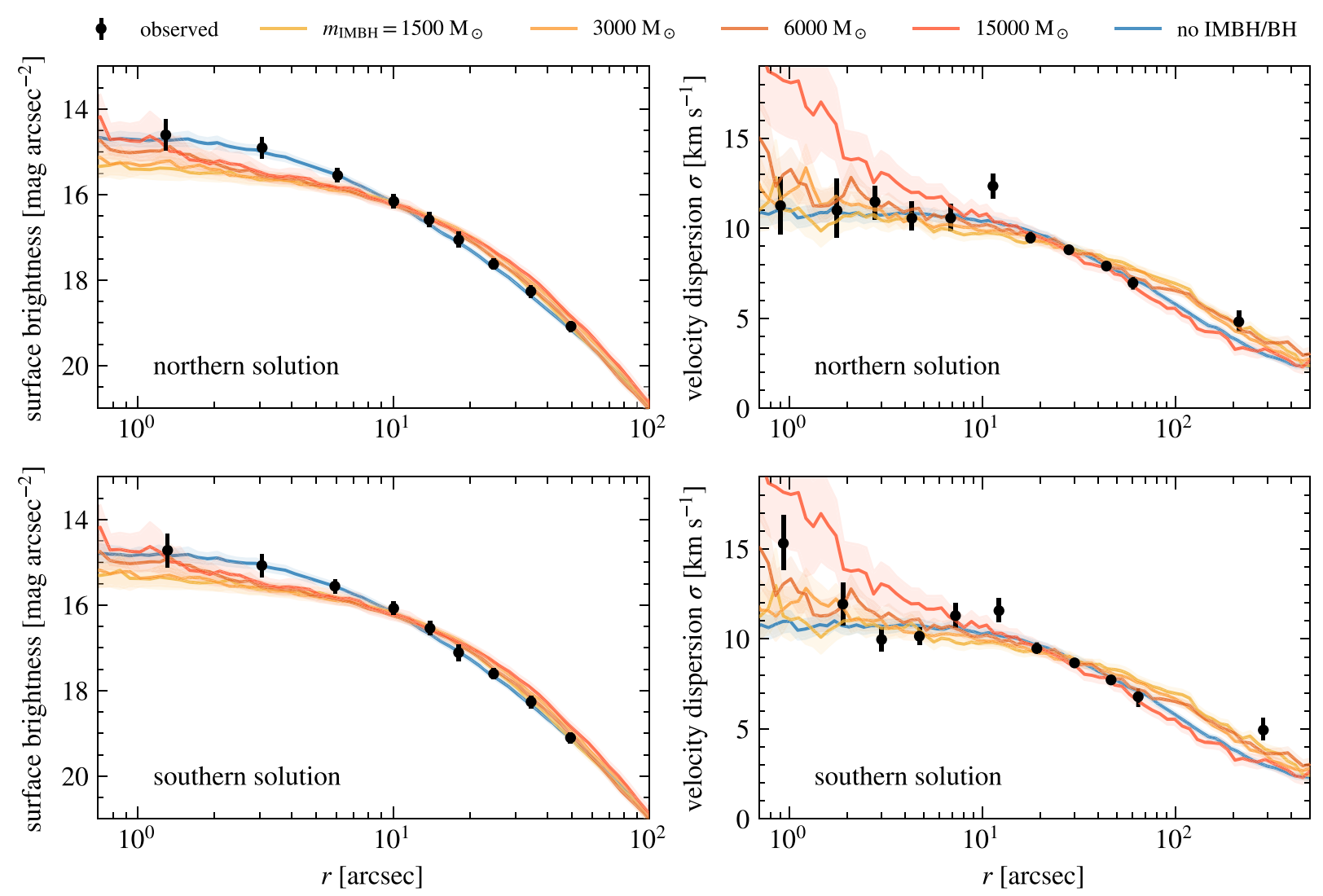

Figure 8. Comparison of the observed surface brightness and the observed velocity dispersion computed from radial bins around the northern and southern centre, respectively, to $N$-body models with an IMBH. See Tables A3 and A4 for more details on the models.

the velocity dispersion profile, while a no-IMBH solution gives $\chi_{r}^{2}=2.25$. Including the fit to the surface brightness profile as well, the reduced $\chi_{r}^{2}$ value is 1.16 for the 2 per cent IMBH model and 1.51 for the 0.5 per cent IMBH model while the no-IMBH model has $\chi_{r}^{2}=1.24$. Hence, the $N$-body models confirm the results of the Jeans modelling that an IMBH of a mass of around $6000 \mathrm{M}_{\odot}$ provides an acceptable fit for M80 if the southern solution is adopted as the density centre. A no-IMBH model does however provide a good fit around this centre as well. Around the northern centre, we find similar fit results. The no-IMBH solution provides the best fit but the IMBH models result only in slightly worse fits and are also acceptable solutions. Again, the surface brightness profile is better reproduced by a model without any IMBH. Thus, if the northern centre is adopted, an IMBH with a mass of up to about $6000 \mathrm{M}_{\odot}$ is possible as well.

The corresponding fit of models with different retention fractions of stellar-mass black holes is depicted in Fig. 9. We obtain rather poor fits to the surface brightness profile for models with retention fractions of stellar-mass black holes greater than 30 percent. The fits become worse the larger the assumed retention fraction of stellar-mass black holes. This is particularly striking for the surface brightness profile, as all models with significant retention fractions produce a core that is larger than what is observed, in agreement with the prediction that clusters harbouring a considerable number of black holes should have large core radii (Arca Sedda, Askar \& Giersz 2018). We therefore conclude that the initial retention fraction of black holes in M80 was low or that nearly all stellar-mass black holes have been ejected from this cluster.

\section{DISCOVERY OF TWO STARS WITH HIGH RADIAL VELOCITY}

We find two stars very close to the Goldsbury et al. (2010) cluster centre (with a distance less than about 1 arcsec, see Fig. 6) with a high radial velocity relative to the Solar system barycentre: a mainsequence star with $v=(88 \pm 7) \mathrm{km} \mathrm{s}^{-1}$ at a projected distance of $1.1 \operatorname{arcsec}\left(\mathrm{RA}=16^{\mathrm{h}} 17^{\mathrm{m}} 2.345\right.$, Dec. $\left.=-22^{\circ} 58^{\prime} 33^{\prime \prime} .33\right)$ and $\mathrm{a}$ subgiant star with $v=(101 \pm 4) \mathrm{km} \mathrm{s}^{-1}$ at a projected distance of $0.4 \operatorname{arcsec}\left(\mathrm{RA}=16^{\mathrm{h}} 17^{\mathrm{m}} 2.414\right.$, Dec $\left.=-22^{\circ} 58^{\prime} 33^{\prime \prime} .51\right)$. While we have two spectra of the main-sequence star and three spectra of the subgiant, we could only derive one and two useful radial velocities respectively due to the low signal-to-noise of the remaining spectra. The radial velocity of both stars is well above the central escape velocity of M80 (41.4 $\mathrm{km} \mathrm{s}^{-1}$; Baumgardt \& Hilker 2018).

High-velocity stars have been reported in three other clusters: in NGC 2808 (Lützgendorf et al. 2012), in M3 (Gunn \& Griffin 1979), and in 47 Tuc (Meylan, Dubath \& Mayor 1991). In these cases, two stars were detected with a radial velocity a few times the velocity dispersion above or below the mean cluster velocity but below the central escape velocity of the respective cluster.

The high radial velocity of these two stars can be explained in several ways.

\subsection{Foreground star}

One or both stars could be foreground stars instead of cluster members. Using the Besançon model (Robin et al. 2003) of the region 

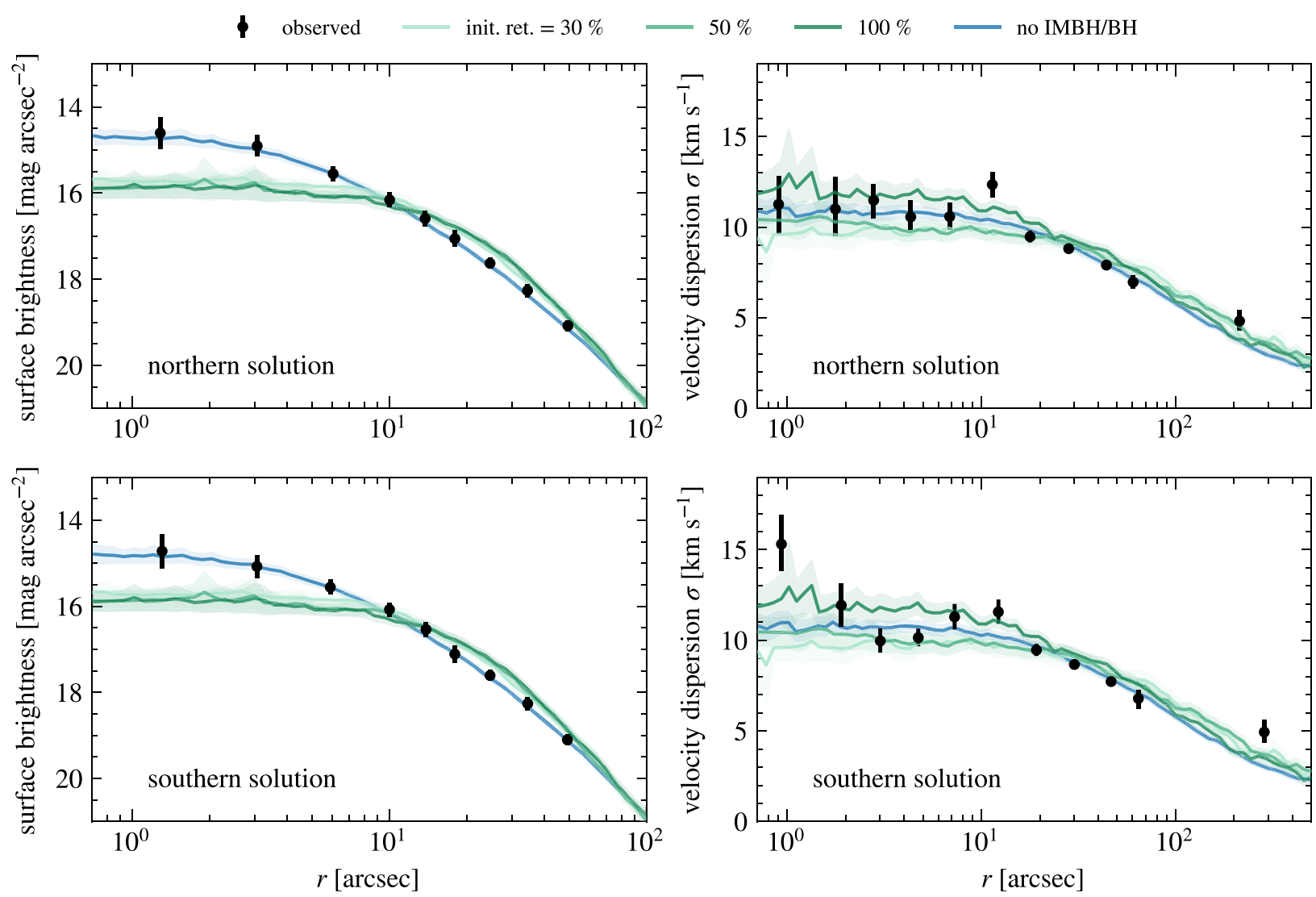

Figure 9. Comparison of the observed surface brightness and the observed velocity dispersion computed from radial bins around the northern and southern centre, respectively, to $N$-body models with stellar-mass BHs. The $N$-body models have different initial retention fractions for stellar-mass BHs (see Tables A3 and A4 for more details on the models).

close to M80, we estimate that about 0.7 per cent of all foreground stars have $-2.25<[\mathrm{Fe} / \mathrm{H}]<-1.75,|v|>80 \mathrm{~km} \mathrm{~s}^{-1}$ and a $\mathrm{V}$ brightness above $21 \mathrm{mag}$. As we find $n=25$ non-member stars in the central 6 arcsec, the probability of having one high-velocity non-member star in this region is 16 percent, and it is 5 percent for the central 1.5 arcsec. The probability that two or more such stars are present is about 1 percent and 0.04 per cent, respectively. In order to further investigate the membership of the fast-moving stars, we analysed two sets of HST observations, one obtained in 2006 (HST proposal ID 10775, PI: Sarajedini) and one taken in 2012 (HST proposal ID 12605, PI: Piotto). We analysed each data set using DOLPHOT (Dolphin 2000, 2016) and transformed the HST coordinates into the ICRS reference frame by cross-matching the stellar coordinates against those of the Gaia EDR3 catalogue (Gaia Collaboration 2021). For each data set, we performed an epoch transformation of the Gaia coordinates back to the epoch when the HST observations were taken, using the measured Gaia proper motions of the stars. Comparison of two sets of stellar coordinates obtained from the cross-matching then allows us to calculate the absolute proper motions of the stars. We obtain a mean cluster motion of $\left(\mu_{\alpha} *, \mu_{\delta}\right)=(-3.03 \pm 0.05,-5.61 \pm 0.05) \mathrm{mas}_{\mathrm{yr}}^{-1}$, in good agreement with the proper motion found by Vasiliev \& Baumgardt (2021) from the Gaia EDR3 data directly. For the fast-moving SGB star, we find a mean proper motion of $\left(\mu_{\alpha} *, \mu_{\delta}\right)=(-3.37 \pm 0.45$, $-5.87 \pm 0.45)$ mas $\mathrm{yr}^{-1}$, well within the range of proper motions that we obtain for the other member stars and fully compatible with a cluster membership of this star. We therefore consider it likely that the SGB star is a cluster member. Unfortunately, we are not able to determine a proper motion for the main-sequence star.

\subsection{Binary star}

The orbital motion of stars in a binary system could be the source for the high radial velocity. Since we only have two radial velocities $\left(104.9 \pm 6.4\right.$ and $\left.99.1 \pm 5.4 \mathrm{~km} \mathrm{~s}^{-1}\right)$ taken about 10 months apart for the subgiant star and only one for the main-sequence star, we cannot entirely exclude variations in the radial velocity. However, we note that our measurements are consistent with a constant radial velocity. Plausible binary system configurations that would produce a velocity amplitude of about $90 \mathrm{~km} \mathrm{~s}^{-1}$ containing a star with a mass of $0.75 \mathrm{M}_{\odot}$ are a short-period system with a low-mass star, a system with a compact host (white dwarf, neutron star, stellar-mass black hole) with a period of several days, or a system with an IMBH as host with a period of several years. To estimate the number of suitable binary systems, we use a binary fraction of 5 per cent estimated from our data, comparable to the low binary fraction of less than about 5 per cent in this cluster (see Milone et al. 2012; Ji \& Bregman 2015). This value is higher than the 2 percent of stars removed from our sample because of radial velocity variations (Section 3.4) since it is corrected for incompleteness due to a limited number of epochs and low-velocity variations. Assuming that 1 percent of all binary systems have a suitable configuration, we expect 0.5 of 1000 stars to be in such stellar binary systems. If one of the stars is in a bound orbit around an IMBH with a mass of $4000 \mathrm{M}_{\odot}$, its orbital distance must be less than $2 m_{\mathrm{IMBH}} G / v^{2}$ which corresponds to $0.1 \operatorname{arcsec}$ at the 
distance of M80. This also excludes the possibility that both stars are bound to the same IMBH.

\subsection{Radial velocity outlier}

Since the radial velocities of both stars are about 40 and $50 \mathrm{~km} \mathrm{~s}^{-1}$ greater than the cluster escape velocity of $41.4 \mathrm{~km} \mathrm{~s}^{-1}$ (Baumgardt \& Hilker 2018), the stars are not gravitationally bound to the cluster.

\subsection{Accelerated by interaction}

Interactions between binary systems with a single stellar-mass black hole can scatter one of the stars in the binary system and accelerate it to high velocities (e.g. Lützgendorf et al. 2012). We expect the probability of this process to be proportional to the number of stellarmass black holes in the cluster, which is low according to the bestfitting $N$-body models. Similarly, an IMBH, if present, should be able to scatter stars in the same way as a stellar-mass black hole.

In conclusion, the mechanism leading to the high radial velocities is not obvious. One of the most likely explanations, the Kepler motion in a binary system, could easily be confirmed or rejected with a few additional measurements.

\section{DISCUSSION}

Our MCMC sampling reveals the existence of two possible solutions for the centre offsets of the Jeans model of M80: the northern solution has the centre close to the photometric centres from the literature and it does not need an IMBH to describe the observed kinematics, and a southern solution with a centre at a distance of about 2.4 arcsec from the northern one that needs an IMBH with a mass of $4600_{-1400}^{+1700} \mathrm{M}_{\odot}$.

The dynamical centre of the southern solution is at a distance of about $2-3$ arcsec from the photometric centres in the literature. A distinct dynamical centre would indicate a perturbed system, possibly caused by an IMBH. We note that a distinct dynamical centre would be inconsistent with the assumption that the cluster can be described with a Jeans model where photometric and dynamical centre are the same by construction. We try to minimize this discrepancy by choosing an MGE profile constructed for the current centre in each iteration. Physically, the position of the IMBH is not expected to be identical with the photometric or dynamical centre. $N$-body models predict that the IMBH will wander inside a sphere with a characteristic radius, the wandering radius $r_{w}$, around the centre. de Vita, Trenti \& MacLeod (2018) analysed $N$-body models and found a scaling relation for this radius that depends on a number of cluster parameters (de Vita et al. 2018, equation 17). Using values for the core density and radius from Baumgardt \& Hilker (2018) and assuming a mean stellar mass in the core of $0.65 \mathrm{M}_{\odot}$, we find $r_{w} \approx 0.4$ arcsec for an IMBH mass of about $3000-4600 \mathrm{M}_{\odot}$. This radius is less than the distance between photometric and dynamical centre of the southern solution, indicating that the wandering motion cannot be the explanation for the observed offset. The sphere of influence of an IMBH with a mass of $4600 \mathrm{M}_{\odot}$ has a radius of $0.09 \mathrm{pc}$ (Peebles 1972), corresponding to 1.8 arcsec at the distance of M80.

Lützgendorf et al. (2013) found a $1 \sigma$ upper limit of $800 \mathrm{M}_{\odot}$ for an IMBH in M80 using data from the integral-field spectrograph FLAMES/ARGUS. This value is well below all upper limits we derived. Lützgendorf et al. (2013) calculated their own position of the cluster centre (see Fig. 6) and did not obtain the velocity dispersion by analysing spectra of individual stars, instead they combined unresolved spectra in radial bins. Their velocity dispersion profile is systematically lower in the cluster centre than ours: while our profile (Fig. 4) is above $10 \mathrm{~km} \mathrm{~s}^{-1}$ for all radii below 10 arcsec (even after accounting for the uncertainty in the determination of the correct centre), their profile stays below $10 \mathrm{~km} \mathrm{~s}^{-1}$. As discussed by Bianchini et al. (2015), there are possible systematic errors in both methods, integrated-light spectroscopy and single-star kinematics. However, a common criticism regarding the latter is that the dispersion would be biased towards low values because of contamination from unresolved stars. The fact that our dispersion measurements are above those by Lützgendorf et al. (2013) suggests that our method is not affected by this. Our IMBH mass upper limit of $3000 \mathrm{M}_{\odot}$ for the northern solution is below the IMBH mass estimate of $(3.63 \pm 0.95) \times 10^{3} \mathrm{M}_{\odot}$ predicted from Monte Carlo models (Arca Sedda et al. 2019), while the median IMBH mass of the southern solution agrees with their result. Estimates for the mass of an IMBH in M80 based on the $M_{\bullet}-\sigma$ correlation and similar correlations range from 1000 to $2610 \mathrm{M}_{\odot}$ (Safonova \& Shastri 2010, table 6).

We assumed that the cluster kinematics can be described by an isotropic model (see Section 4.1). As Zocchi, Gieles \& HénaultBrunet (2017) point out, radially anisotropic models of NGC 5139 show an increase in the central velocity dispersion similar to that due to the influence of an IMBH. If our assumption about isotropy is not satisfied in M80, our isotropic model would overestimate the IMBH mass. Since the cluster has a ratio of age to relaxation time greater than ten, the $N$-body models used in Lützgendorf et al. (2011) imply that the cluster centre is isotropic.

The comparison to $N$-body models performed in Section 5 suggests that the observed steep surface brightness profile of the cluster is better explained by models without IMBH. The binned surface brightness profile presented in Figs 4, 8, and 9 is derived from the photometry of Dalessandro et al. (2018) that is based on HST observations. This profile is about $0.5-0.8 \mathrm{mag} \operatorname{arcsec}^{-2}$ brighter in the central bins compared to the profile of Noyola \& Gebhardt (2006) and about 1-1.3 mag $\operatorname{arcsec}^{-2}$ brighter than the profiles of Trager, King \& Djorgovski (1995) computed from ground-based photometry. Figs 8 and 9 indicate that the other surface brightness profiles with lower central values is less well fitted by the no-IMBH model and better fitted by the models with an IMBH. An important difference between the two aforementioned surface brightness profiles and the one derived in this work is that the former are based on actual brightness measurements, whereas we adopted star counts. Profiles based on star counts are robust against shot noise effects caused by individual bright stars, yet require complete photometry even in the crowded cluster centres. The availability of ACS-HRC data makes us confident that our results are not affected by incompleteness. Note that we only included stars brighter than $F 435 W<19.6$ mag, i.e. about the main-sequence turn-off, when determining the number density profiles.

Ultimately, our models cannot answer the question which of the two solutions for the cluster centre is to be preferred, and therefore whether an IMBH exists in M80. In light of the better agreement with the photometric determinations of the cluster centre, the northern solution seems the more likely one. However, our models do include a prior which favours solutions in agreement with the photometric centres. Hence, in case the true cluster centre coincides with the photometric estimates (and our northern solution), the high-velocity dispersion around the centre of the southern solution is leading our Jeans models astray. As mentioned earlier, we verified that no individual high-velocity stars are responsible for the occurrence of the southern solution. Still, we cannot exclude that some of our model assumptions, such as a Gaussian line-of-sight velocity distribution 
or axisymmetry, are violated by the actual kinematics in the centre of M80.

\section{CONCLUSIONS}

We used spectra obtained with state-of-the-art adaptive optics of the central region in M80 to analyse kinematic properties of the cluster. We built on an axisymmetric Jeans model used previously for this cluster by Kamann et al. (2020) and introduced additional parameters to describe a hypothetical IMBH and offsets between the photometric centre and the kinematic centre. The parameter samples of our Jeans model show a bimodal distribution in the centre offsets: a third of all samples are part of a northern solution with a centre close to the known photometric centres, the other two-thirds are part of the southern solution with a centre at a distance of about 2.4 arcsec from the northern centre. While most parameters do not show significant differences between the two solutions, the distribution of mass of the central IMBH is different. Around the northern centre, we find a distribution with a peak below $1000 \mathrm{M}_{\odot}$ that quickly decreases with increasing IMBH mass. The 90 per cent upper limit is $3000 \mathrm{M}_{\odot}$. The IMBH mass distribution of the southern solution has a median and an $1 \sigma$ uncertainty of $4600_{-1400}^{+1700} \mathrm{M}_{\odot}, 90$ per cent of all samples are below $6800 \mathrm{M}_{\odot} . N$-body models support the existence of an IMBH in this cluster with a mass of up to $6000 \mathrm{M}_{\odot}$ although models without an IMBH provide a better fit to the observed surface brightness profile. They further indicate that the cluster has lost nearly all stellar-mass black holes. The overall radial profiles of the mass-to-light ratio and the rotation velocity agree with the previous analysis of this cluster (Kamann et al. 2020). While the part outside the cluster core clearly rotates, it is not clear whether the core of the cluster is rotating. Our analysis is consistent with no rotation in the centre but the uncertainty in the position of the cluster centre prohibits any definitive conclusion. We discussed the detection of two central stars with radial velocities clearly above the escape velocity of the cluster. Their high velocities could be explained if one or both stars belong to the Milky Way population instead to M80, if they are caused by binary motion, or if one or both stars were accelerated by interactions between a binary system and a stellar-mass or intermediate-mass black hole. Of these possibilities, the periodic change of radial velocity due to binary motion can be confirmed or ruled out by future observations.

Proper motion data of stars in the cluster centre could complement the radial velocities used in this study. Deep radio and X-ray observations of the cluster centre could lead to further insights about a possible IMBH in M80, similar to other clusters (Tremou et al. 2018). Finally, detailed studies of the stellar-mass function or the binary fraction within the core radius of M80 could be used to understand the amount of mass segregation present near the centre of M80. As argued by some authors, e.g. Gill et al. (2008), an IMBH would strongly reduce the amount of mass segregation expected near the centre. In this respect, the finding of Dalessandro et al. (2018) of differences in the concentrations of the three chemically distinct populations discovered in M80 might be considered as evidence against an IMBH.

\section{ACKNOWLEDGEMENTS}

We thank the referee Glenn van de Ven for his constructive suggestions and comments that helped to improve the paper. FG, SD, BG, and TOH acknowledge funding from the Deutsche Forschungsgemeinschaft (grant DR 281/35-1 and KA 4537/2-1) and from the German Ministry for Education and Science (BMBF-Verbundforschung) through grants 05A14MGA, 05A17MGA, 05A14BAC, 05A17BAA, and 05A20MGA. SK gratefully acknowledges funding from UKRI in the form of a Future Leaders Fellowship (grant no. MR/T022868/1). The authors acknowledge the French National Research Agency (ANR) for supporing this work through the ANR APPLY (grant ANR-19-CE31-0011) coordinated by B. Neichel. The work was supported by the North-German Supercomputing Alliance (HLRN).

\section{DATA AVAILABILITY}

The data underlying this article are available in the Göttingen Research Online repository, at https://doi.org/10.25625/VCNHOR. The raw exposures used in this article are available under their Programme ID (Table A1) in the ESO Archive, at https://archive.eso.org.

\section{REFERENCES}

Aarseth S. J., 1999, PASP, 111, 1333

Abbate F., Possenti A., Colpi M., Spera M., 2019, ApJ, 884, L9

Abbott R. et al., 2020, ApJ, 900, L13

Anderson J. et al., 2008, AJ, 135, 2055

Arca Sedda M., Askar A., Giersz M., 2018, MNRAS, 479, 4652

Arca Sedda M., Askar A., Giersz M., 2019, preprint (arXiv:1905.00902)

Bacon R. et al., 2010, in McLean I. S., Ramsay S. K., Takami H., eds, Proc. SPIE Conf. Ser. Vol. 7735, Ground-Based and Airborne Instrumentation for Astronomy III. SPIE, Bellingham, p. 773508

Baumgardt H., 2017, MNRAS, 464, 2174

Baumgardt H., Hilker M., 2018, MNRAS, 478, 1520

Baumgardt H. et al., 2019, MNRAS, 488, 5340

Baumgardt H., Sollima A., Hilker M., 2020, Publications of the Astronomical Society of Australia, 37, e046

Beers T. C., Flynn K., Gebhardt K., 1990, AJ, 100, 32

Bellini A. et al., 2014, ApJ, 797, 115

Bianchini P., Norris M. A., van de Ven G., Schinnerer E., 2015, MNRAS, 453,365

Cappellari M., 2008, MNRAS, 390, 71

Dalessandro E. et al., 2018, ApJ, 859, 15

de Boer T. J. L., Gieles M., Balbinot E., Hénault-Brunet V., Sollima A., Watkins L. L., Claydon I., 2019, MNRAS, 485, 4906

de Vita R., Trenti M., MacLeod M., 2018, MNRAS, 475, 1574

Dolphin A., 2000, PASP, 112, 1383

Dolphin A., 2016, Astrophysics Source Code Library, record ascl:1608.013

Dotter A. et al., 2009, ApJ, 708, 698

Ferraro F. R. et al., 2012, Nature, 492, 393

Fétick R. J. L. et al., 2019, A\&A, 628, A99

Foreman-Mackey D., Hogg D. W., Lang D., Goodman J., 2013, PASP, 125, 306

Gaia Collaboration, 2021, A\&A, 649, A1

Gieles M., Balbinot E., Yaaqib R., Henault-Brunet V., Zocchi A., Peuten M., Jonker P. G., 2018, MNRAS, 473, 4832

Giersz M., Leigh N., Hypki A., Lützgendorf N., Askar A., 2015, MNRAS, 454,3150

Giesers B. et al., 2019, A\&A, 632, A3

Gill M., Trenti M., Miller M. C., van der Marel R., Hamilton D., Stiavelli M., 2008, ApJ, 686, 303

Goldsbury R., Richer H. B., Anderson J., Dotter A., Sarajedini A., Woodley K., 2010, AJ, 140, 1830

Greene J. E., Strader J., Ho L. C., 2020, ARA\&A, 58, 257

Gunn J. E., Griffin R. F., 1979, AJ, 84, 752

Harris W. E., 1996, AJ, 112, 1487

Hénault-Brunet V., Gieles M., Strader J., Peuten M., Balbinot E., Douglas K. E. K., 2020, MNRAS, 491, 113

Hogg D. W., Foreman-Mackey D., 2018, ApJS, 236, 11

Husser T.-O. et al., 2016, A\&A, 588, A148

Jalali B., Baumgardt H., Kissler-Patig M., Gebhardt K., Noyola E., Lützgendorf N., Zeeuw P. T. d., 2012, A\&A, 538, A19

Ji J., Bregman J. N., 2015, ApJ, 807, 32 
Kamann S., Wisotzki L., Roth M. M., 2013, A\&A, 549, A71

Kamann S. et al., 2018, MNRAS, 473, 5591

Kamann S. et al., 2020, MNRAS, 492, 966

Kiziltan B., Baumgardt H., Loeb A., 2017, Nature, 542, 203

LIGO Scientific Collaboration, Virgo Collaboration, 2020, Phys. Rev. Lett., 125,101102

Lützgendorf N., Kissler-Patig M., Noyola E., Jalali B., de Zeeuw P. T., Gebhardt K., Baumgardt H., 2011, A\&A, 533, A36

Lützgendorf N. et al., 2012, A\&A, 543, A82

Lützgendorf N. et al., 2013, A\&A, 552, A49

MacLeod M., Trenti M., Ramirez-Ruiz E., 2016, ApJ, 819, 70

Mann C. R. et al., 2019, ApJ, 875, 1

Meylan G., Dubath P., Mayor M., 1991, ApJ, 383, 587

Milone A. P. et al., 2012, A\&A, 540, A16

Nitadori K., Aarseth S. J., 2012, MNRAS, 424, 545

Noyola E., Gebhardt K., 2006, AJ, 132, 447

Noyola E., Gebhardt K., Kissler-Patig M., Lützgendorf N., Jalali B., Zeeuw P. T. d., Baumgardt H., 2010, ApJ, 719, L60

Peebles P. J. E., 1972, ApJ, 178, 371

Portegies Zwart S. F., Baumgardt H., Hut P., Makino J., McMillan S. L. W., 2004, Nature, 428, 724
Rizzuto F. P., Naab T., Spurzem R., Giersz M., Ostriker J. P. et al., 2021, MNRAS, 501, 5257

Robin A. C., Reylé C., Derrière S., Picaud S., 2003, A\&A, 409, 523

Safonova M., Shastri P., 2010, Ap\&SS, 325, 47

Sarajedini A. et al., 2007, AJ, 133, 1658

Sollima A., Baumgardt H., Hilker M., 2019, MNRAS, 485, 1460

Trager S. C., King I. R., Djorgovski S., 1995, AJ, 109, 218

Tremou E. et al., 2018, ApJ, 862, 16

Usher C., Kamann S., Gieles M., Hénault-Brunet V., Dalessandro E., Balbinot E., Sollima A., 2021, MNRAS,503, 1680

van den Bosch R., de Zeeuw T., Gebhardt K., Noyola E., van de Ven G., 2006 ApJ, 641, 852

Vasiliev E., Baumgardt H., 2021, MNRAS, 505, 5978

Watkins L. L., van de Ven G., den Brok M., van den Bosch R. C. E., 2013, MNRAS, 436, 2598

Watkins L. L., Marel R. P. v. d., Bellini A., Anderson J., 2015, ApJ, 803, 29

Weilbacher P. M. et al., 2020, A\&A, 641, A28

Zocchi A., Gieles M., Hénault-Brunet V., 2017, MNRAS, 468, 4429

Zocchi A., Gieles M., Hénault-Brunet V., 2019, MNRAS, 482, 4713

\section{APPENDIX A: ADDITIONAL FIGURES AND TABLES}

Table A1. MUSE observations of NGC 6093

\begin{tabular}{|c|c|c|c|c|}
\hline Pointing & Obs. date & Inst. mode & Exp. time & Prog. ID \\
\hline \multirow[t]{3}{*}{01} & 2015-05-11 07:56:56 & WFM & $3 \times 200 s$ & 095.D-0629 \\
\hline & 2017-04-23 09:10:55 & WFM & $3 \times 200 \mathrm{~s}$ & 099.D-0019 \\
\hline & 2019-05-04 06:44:49 & WFM-AO & $3 \times 200 s$ & 0103.D-0204 \\
\hline \multirow[t]{4}{*}{02} & 2015-05-11 08:12:39 & WFM & $3 \times 200 s$ & 095.D-0629 \\
\hline & 2017-02-01 09:11:41 & WFM & $3 \times 200 s$ & 098.D-0148 \\
\hline & 2017-04-23 09:29:43 & WFM & $3 \times 200 s$ & 099.D-0019 \\
\hline & 2019-05-04 07:01:07 & WFM-AO & $3 \times 200 s$ & 0103.D-0204 \\
\hline \multirow[t]{4}{*}{03} & 2015-05-11 08:42:29 & WFM & $3 \times 200 s$ & 095.D-0629 \\
\hline & 2015-05-11 08:58:28 & WFM & $3 \times 200 \mathrm{~s}$ & 095.D-0629 \\
\hline & 2017-04-26 04:22:07 & WFM & $3 \times 200 s$ & 099.D-0019 \\
\hline & 2019-05-04 07:25:53 & WFM-AO & $3 \times 200 s$ & 0103.D-0204 \\
\hline \multirow[t]{3}{*}{04} & 2015-05-11 09:14:19 & WFM & $3 \times 200 s$ & 095.D-0629 \\
\hline & 2017-04-26 04:37:08 & WFM & $2 \times 200 \mathrm{~s}$ & 099.D-0019 \\
\hline & 2019-05-04 07:42:12 & WFM-AO & $3 \times 200 \mathrm{~s}$ & 0103.D-0204 \\
\hline \multirow[t]{2}{*}{91} & 2019-05-04 09:40:10 & NFM-AO & $4 \times 600 s$ & 0103.D-0204 \\
\hline & 2020-02-24 08:45:05 & NFM-AO & $4 \times 600 s$ & 0104.D-0257 \\
\hline
\end{tabular}

Table A2. Priors used in the Jeans model MCMC.

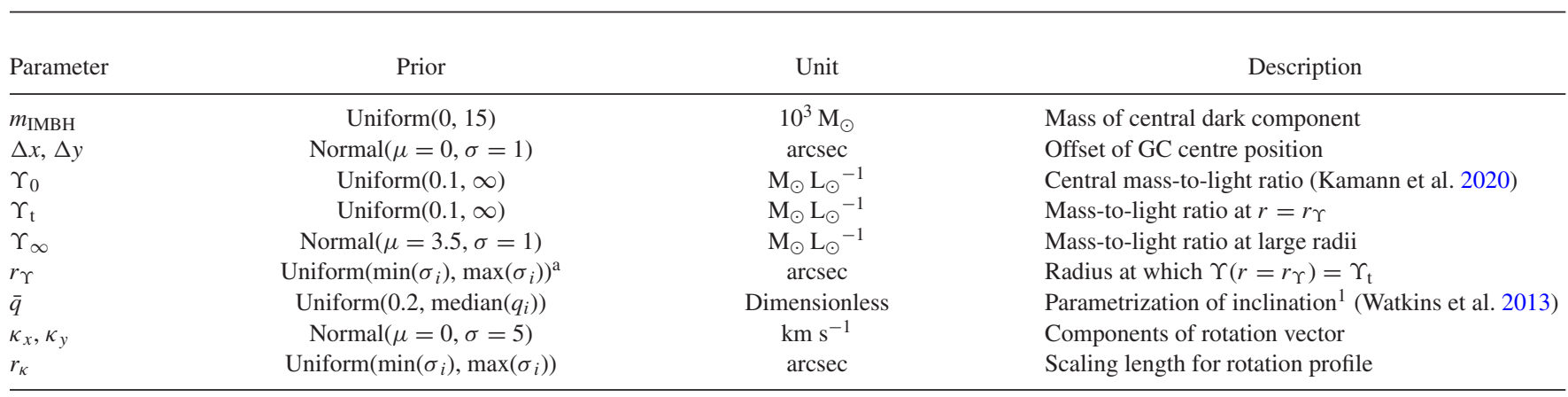

${ }^{a} \sigma_{i}$ and $q_{i}$ are the width and the axial ratio of the $i$-th component of the MGE. 
Table A3. Fit statistics for $N$-body models around the northern centre. 'RV' refers to fits of the binned radial velocities, 'SB' to fits of the surface brightness profile, and 'total' to simultaneous fits to both.

\begin{tabular}{lcccc}
\hline & $\begin{array}{c}\text { SBH retention frac. } \\
\text { (per cent) }\end{array}$ & $\chi_{r}^{2} \mathrm{RV}$ & $\chi_{r}^{2} \mathrm{SB}$ & $\chi_{r}^{2}$ total \\
\hline 0 & 10 & 1.23 & 0.78 & 0.93 \\
0 & 30 & 2.43 & 1.26 & 1.65 \\
0 & 50 & 1.79 & 1.57 & 1.93 \\
0 & 100 & 1.16 & 1.68 & 2.38 \\
1500 & 10 & 1.94 & 1.56 & 1.66 \\
3000 & 10 & 1.68 & 1.42 & 1.49 \\
6000 & 10 & 1.14 & 1.07 & 1.09 \\
15000 & 10 & 2.10 & 1.33 & 1.54 \\
\hline
\end{tabular}

Table A4. Similar to Table A4 but for $N$-body models around the southern centre.

\begin{tabular}{lcccc}
\hline$m_{\mathrm{IMBH}}\left(\mathrm{M}_{\odot}\right)$ & $\begin{array}{c}\text { SBH retention frac. } \\
\text { (per cent) }\end{array}$ & $\chi_{r}^{2} \mathrm{RV}$ & $\chi_{r}^{2} \mathrm{SB}$ & $\chi_{r}^{2}$ total \\
\hline 0 & 10 & 2.25 & 0.94 & 1.24 \\
0 & 30 & 3.35 & 1.15 & 1.82 \\
0 & 50 & 2.34 & 1.58 & 2.04 \\
0 & 100 & 1.77 & 1.33 & 2.51 \\
1500 & 10 & 2.00 & 0.97 & 1.51 \\
3000 & 10 & 2.06 & 1.02 & 1.21 \\
6000 & 10 & 1.54 & 1.29 & 1.16 \\
15000 & 10 & 1.87 & & 1.44 \\
\hline
\end{tabular}

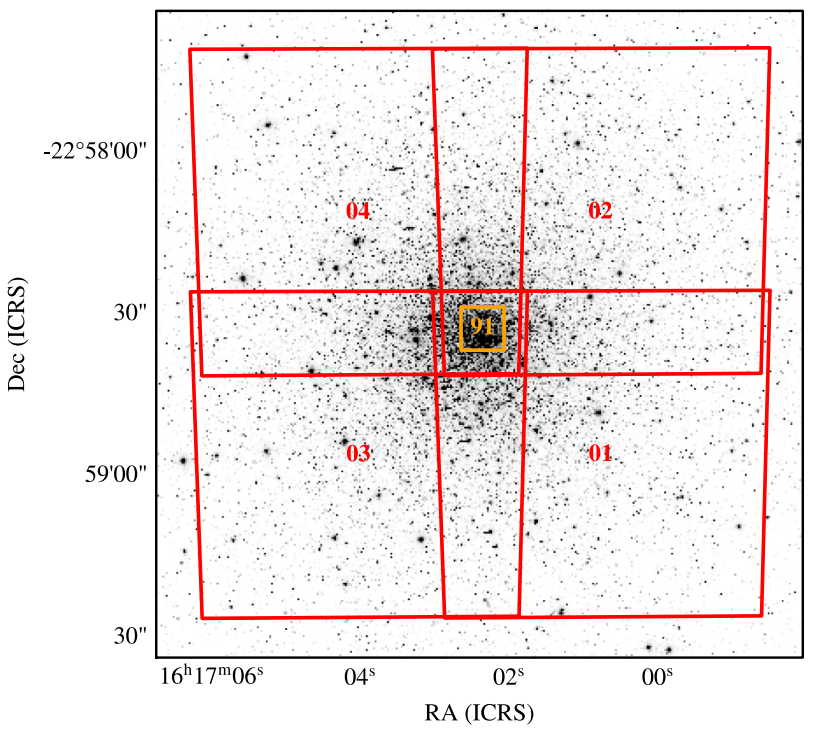

Figure A1. The four MUSE WFM pointings (01-04) and the central NFM pointing (91) on top of the HST ACS image of M80 (Sarajedini et al. 2007; Anderson et al. 2008). 

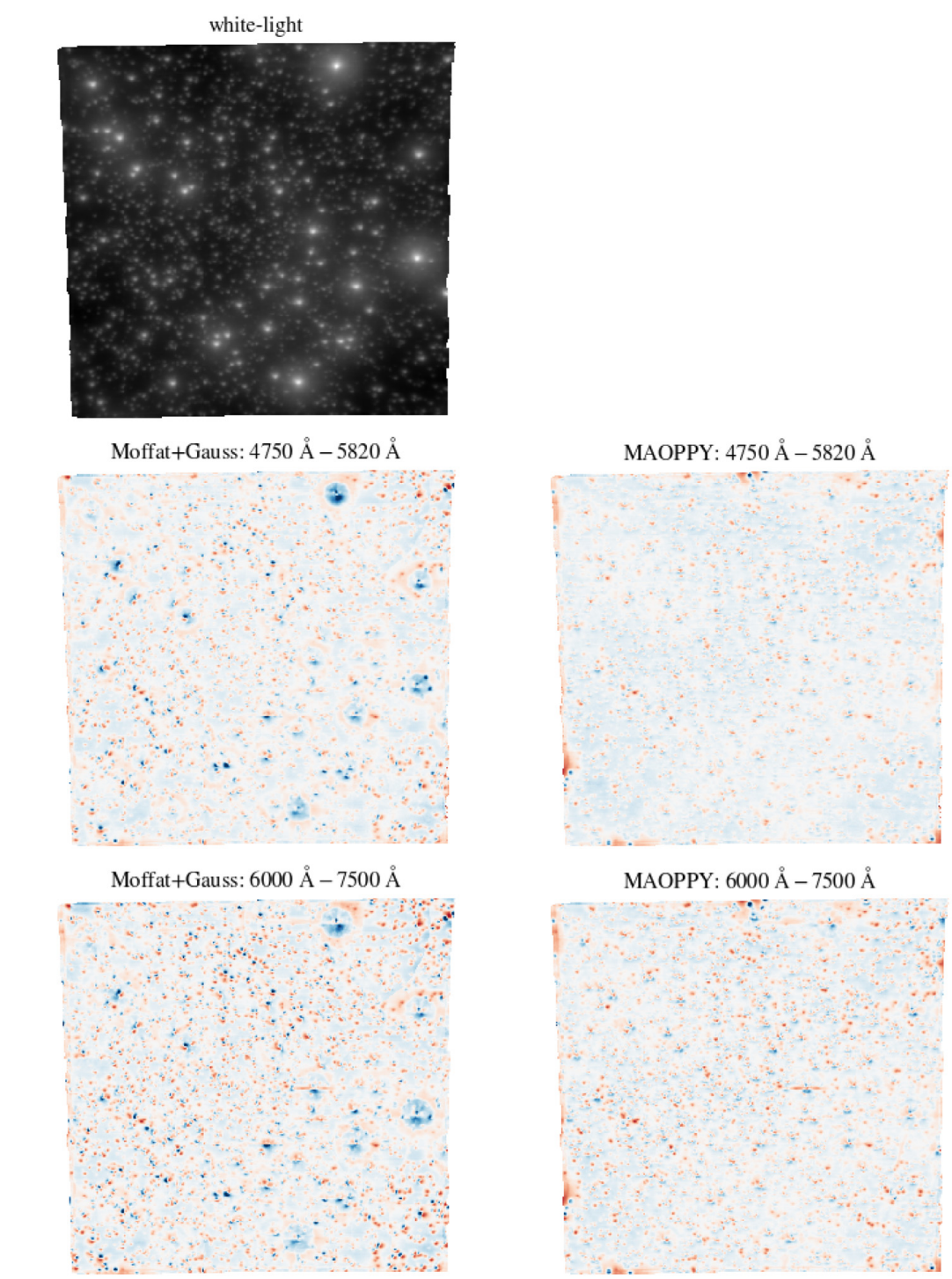

MAOPPY: $6000 \AA-7500 \AA$
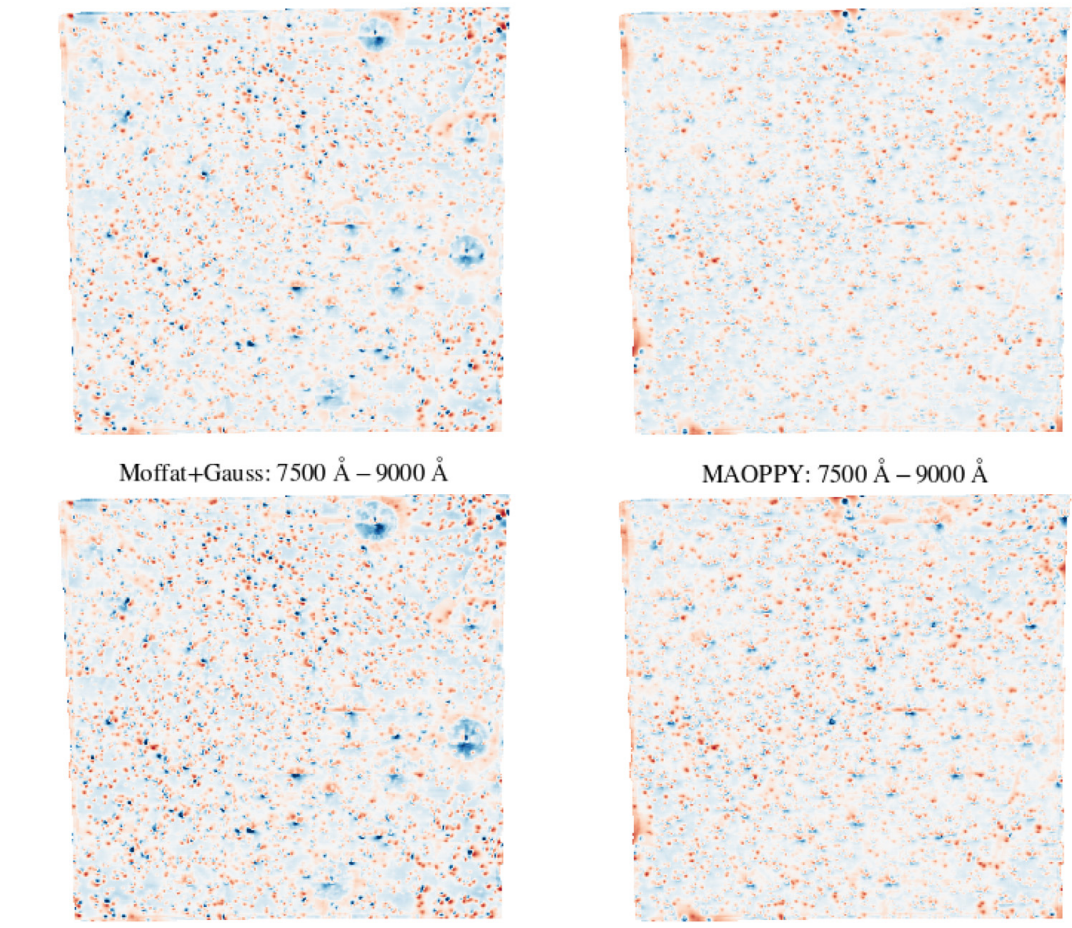

MAOPPY: $7500 \AA-9000 \AA$
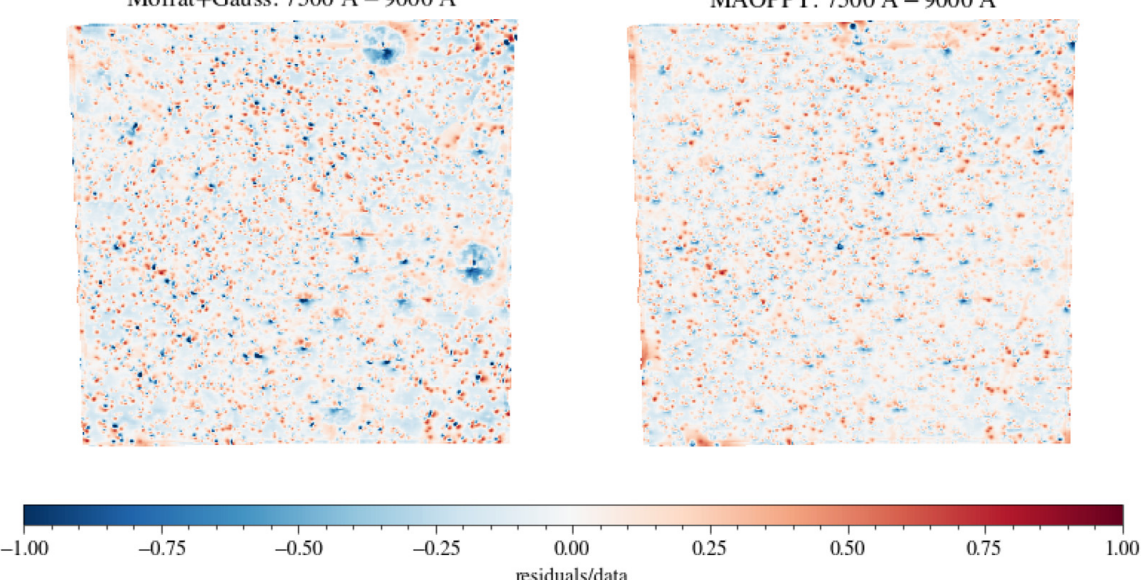

Figure A2. White-light image of an NFM observation and the residuals relative to the original data after subtracting the PSF model (left column: a combination of a Moffat curve with a Gaussian, right column: MAOPPY) and background in three different wavelength ranges. 


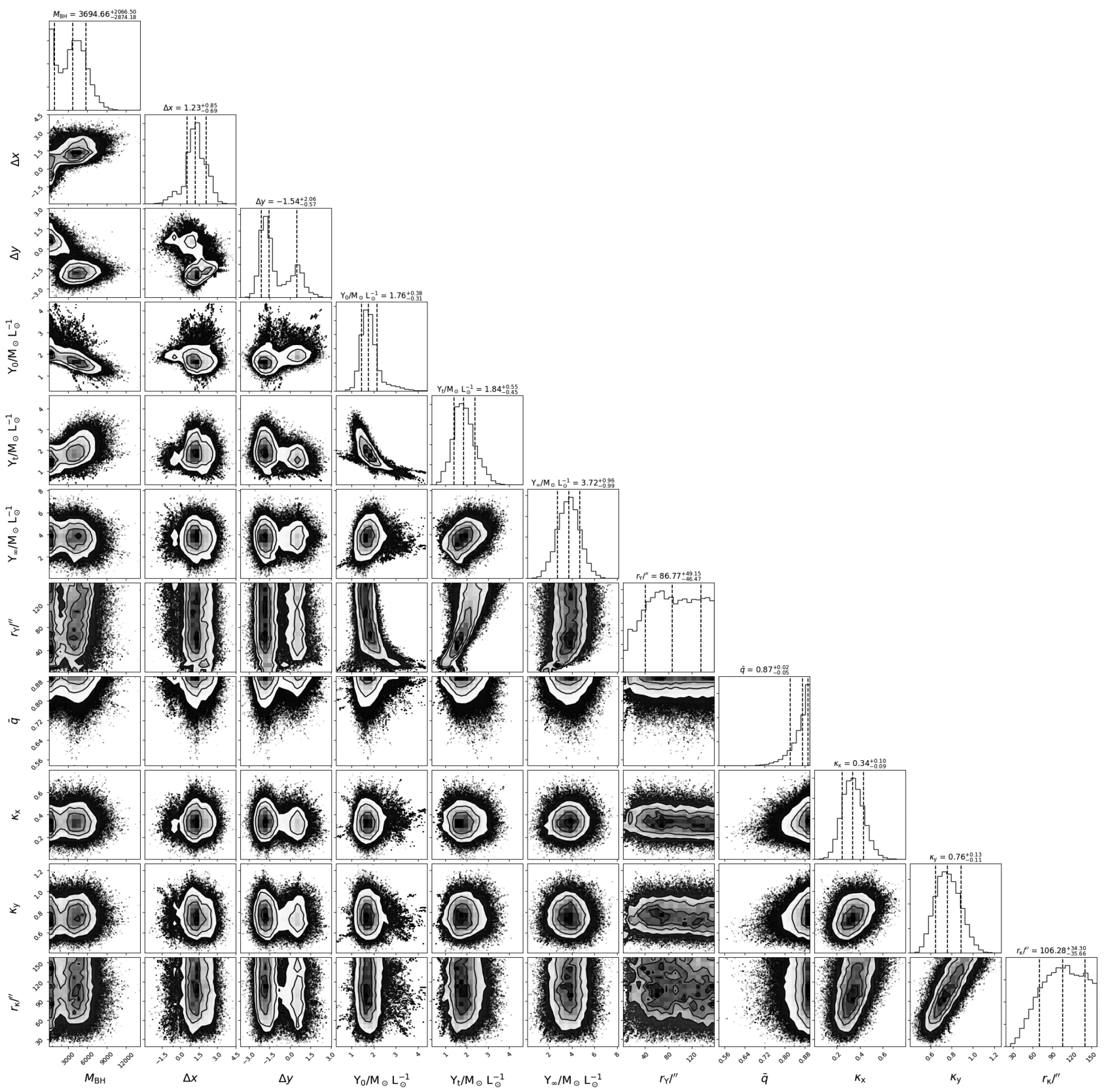

Figure A3. Corner plot of our final MCMC chain. See Table A2 for a description of the parameters. 


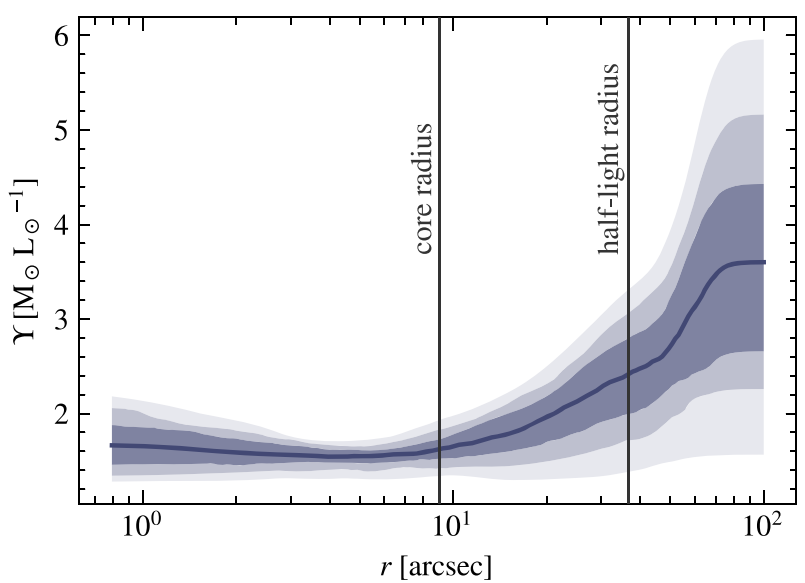

Figure A4. Radial profiles of the mass-to-light ratio $\Upsilon$, the blue lines corresponds to the median, the shaded areas are the $1 \sigma, 2 \sigma$, and $3 \sigma$ limits.

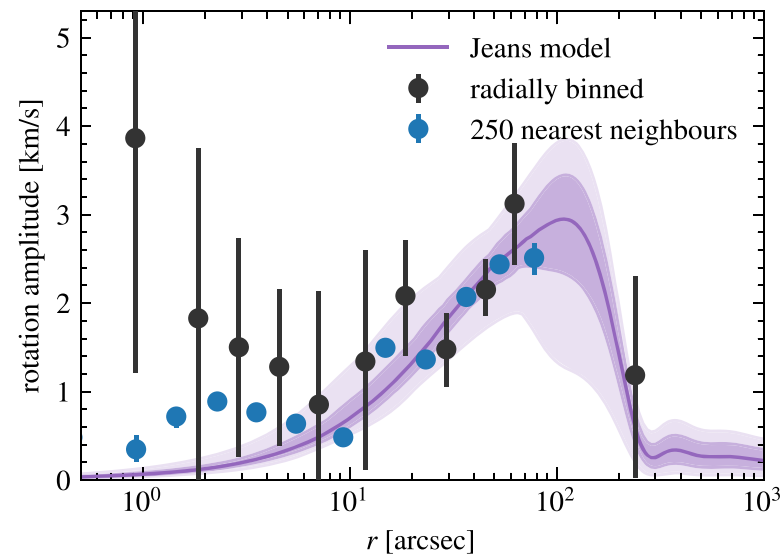

Figure A5. Radial profile of the rotation amplitude derived from the Jeans model, from individual radial velocities in radial bins, and from the mean radial velocities of the 250 nearest neighbours of each star.

This paper has been typeset from a $\mathrm{T}_{\mathrm{E}} \mathrm{X} / \mathrm{L} \mathrm{A} \mathrm{T} \mathrm{X}$ file prepared by the author. 


\section{Chapter 3}

\section{Additions to EMISSION-LINE}

\section{ANALYSIS}

Göttgens et al. (2019b, see Chapter A.1 in the Appendix) present a collection of emission-line objects in Galactic GCs found with MUSE data. This catalogue includes binary stars, variable stars, planetary nebulae, known and new cataclysmic variables, sub-subgiants, X-ray counterparts, and unclassifiable stellar sources. While the main algorithm was developed for my Master's thesis and many detected emission-line objects are already included in that thesis, Göttgens et al. (2019b) contains several important additions that I will briefly summarise here.

The paper includes spectra extracted from MUSE observations made between November 2014 and March 2019, about three years more data than the previous analysis (November 2014 until May 2016). NGC 1904, NGC 6218, and NGC 6624 are analysed in addition to the previous sample of clusters.

By combining measured noise properties with artificial emission lines, we derived limits on the emission flux that we can detect as a function of brightness for each cluster. These limits decrease by three orders of magnitude with increasing brightness from 20 mag to 12 mag but only by about one magnitude between clusters at fixed brightness. It is important to estimate the flux limits of such a survey because they can be compared with theoretical predictions of fluxes expected for e.g. planetary nebulae or cataclysmic variables. This allows the distinction between objects that should have been found if they existed (prediction above flux limit) and objects that can not be detected even if they exist (prediction below flux limit).

One main characteristic of our detection method is to discard spectra with a detection significance below a threshold. In order to justify the use of a detection 
threshold, the paper shows that the fraction of true positive detections (spectra marked as emission line candidates with real emission lines) to total detections correlates with the detection significance. It increases linearly from very low values of $5-10 \%$ at the lowest accepted detection significance of 7.5 to $60 \%$ at a significance of about 20.

Many new emission-line objects are listed in Göttgens et al. (2019b) that are not part of the Master's thesis. These include several candidates for subsubgiants and also the CV that caused the Nova T Scorpii observed in 1860 (Sawyer. 1938), one of the two novae observed in a GC.

Although the paper lists nine CVs (two new ones, seven previously known $\mathrm{CVs}$ or candidates in the literature), which is a large number compared to the ten previously spectroscopically confirmed CVs in a GC, it is still low when it is directly compared to the expected number of CVs per GC which is in the order of one hundred. The paper uses the expected brightness distribution from simulation of Belloni et al. (2019) to show that the nine CVs found in our survey data - given the distances, our spectral extraction completeness, and the emission-line detection efficiency - is actually consistent with the expectation of $10 \pm 2 \mathrm{CV}$ detections.

As a by-product of the search of emission-line objects in GCs, we also found spectra from background starburst galaxies. At first, these detections happened by chance when prominent galactic emission lines (from Oxygen or Hydrogen) were redshifted to the rest-frame wavelength of $\mathrm{H} \alpha$ which was the focus of the stellar emission line search. Later, we developed an algorithm that takes the wavelengths of strong emission line candidates found in a spectrum and checks if they are consistent with a at least three lines from a reference list of wavelengths of galactic emission lines and a common redshift. In total, 21 background galaxies could be found in this way, the range in redshifts is from 0.052 to 0.737 . One galaxy is located at a projected distance of only 4.6 arcsec from the centre of NGC 7099, a core-collapsed cluster and its discovery demonstrates the capability to look through GCs with MUSE. 


\section{Chapter 4}

\section{CONCLUSIONS AND OUTLOOK}

\subsection{The small emission nebula in M22}

The small, low-brightness emission nebula discovered in M22 (Section 2.1) is unique among the four known nebulae (all PNe) in GCs. It is also the only nebula we discovered in the area covered by the MUSE GC survey. If there are any additional PNe present, they must be even less luminous - and thus even less massive - or further outside where they would be easy to find by photometry. If this nebula is indeed connected to the PN in M22, GJJC-1, why is it that only this PN (or its central star) has a detached nebula with different spectral properties? GJJC-1 itself is a strange object, as it seems to be hydrogendeficient among several other puzzling features. Perhaps it is not even a PN (see Jacoby et al., 2017, and references therein). With its low brightness, small size, and short distance to the GC centre, it will be hard to observe this nebula with another instrument, e.g. to measure line widths or emission lines outside the MUSE spectral range. As noted by Jacoby et al. (2017), M22 hosts several peculiar objects, including two stellar-mass black holes (Strader et al., 2012) and of course GJJC-1, and it seems we need to add the recently discovered small emission nebula to that list.

\subsection{Is there an IMBH in M80?}

Our dynamical analysis of about 10,000 mean radial velocities in M80, of which about 1000 are from stars within 8 arcsec of the cluster centre, has two possible solutions: in one solution, the cluster centre is roughly where it is expected from photometric determinations and both Jeans models and $N$-body models do not need any IMBH to explain the observed velocity dispersion profile. However, 
a second solution with a dynamical cluster centre roughly two arcsec to the south of the photometric centre has a higher central velocity dispersion which can only be explained by an IMBH with a mass of 3000 to 6000 solar masses. As additional, simpler analyses show, the region of high velocity dispersion around the southern centre is real. Dispersion maps created by spatially binning velocities in a $2 \mathrm{D}$ square grid or with a nearest-neighbour algorithm show the same increase of the velocity dispersion around the southern centre.

If the northern solution is the one that is realised in nature and thus no IMBH exists in M80 (or it has a much lower mass) then what causes the increased velocity dispersion around the southern centre? Given the number of stars, it is unlikely that uncorrected binaries, i.e. binaries that are not removed from our analysis because their amplitude is too low compared to the velocity uncertainties, is the explanation.

If the southern solution is the real one, what causes the IMBH to be about four times as distant from the photometric centre as expected from numerical simulations? Does the axisymmetric Jeans model simplify the real velocity distribution too much? Since $N$-body models also allow for a 6000 -solar-mass IMBH in the southern centre, it seems unlikely that an IMBH detection is an artefact of the axisymmetric Jeans model.

Similar observations and analyses of more GCs would help to see if solutions with off-centre IMBHs are common to GCs suspected of having an IMBH. More observations could also reveal if other GCs, especially those without an expected IMBH, turn out to have small off-centre regions with an increased velocity dispersion compared to the photometric centre. This will be investigated by analysing NFM observations of additional GCs which already have been made or are currently planned.

\subsection{Central high-velocity stars in GCs}

During the analysis of individual radial velocities in M80, we found two stars close to the cluster centre with radial velocities higher than the escape velocity of the cluster. As mentioned in Section 6 in the paper, the origin of the high velocity of these stars is not clear. In particular, given their position, it seems very unlikely that one of them is the companion of an IMBH (MacLeod et al. 2016) since that would be located in the southern centre of the cluster. This observation shows the power of the MUSE NFM to find high-velocity stars in GCs. Multiple well-timed observations can be used to lower the probability that the radial velocity is due to binarity and the spectral analysis, in particular the metallicity, can be used to check if the star is a foreground object. The detection of central stars with a high but variable radial velocity is an independent way to 
find potential IMBHs: by observing the same GC centre multiple times, over a time span of several years, high-velocities stars could be detected and a simple analysis of their radial velocity curves could reveal if they are the companions of an IMBH or ordinary binaries. Detecting IMBH companions in this way seems far easier than using proper motions or astrometric techniques as suggested by Monty et al. (2021) using the planned MAVIS instrument, similar to Sgr A* (Ghez et al. 2008, Genzel et al., 2010). Compared to dynamical modelling with Jeans models, $N$-body codes, or any other model - the detection of IMBHs by finding their companions via radial velocities does not require numerous simplifications or any energy-intensive calculations.

\subsection{Proper-motions from MUSE NFM observations}

Usually, proper motions of individual stars in GCs are derived from multiple high-resolution images taken with HST, e.g. HSTPROMo (Bellini et al., 2017b). Given the low internal velocities in the order of kilometres per second and the typical distances to GCs of several kiloparsecs, the observations must be taken over a time range of several years. The observations also need to be calibrated in a more sophisticated way compared to normal photometric observations to reduce the effect of optical distortions in the field of view.

Since observations with the MUSE NFM of GC centres can have a spatial resolution better than HST-ACS in the wide-field channel (WFC), the question arises if the NFM data together with the HST catalogue positions can be used to determine proper motions. The idea is to use the published catalogue positions from the ACS Survey (Anderson et al. 2008 Sarajedini et al. 2007) as a reference point and the positions measured in the NFM datacubes as additional data points. In our data analysis pipeline, we use PAmpelMuse (Kamann et al. 2013) to extract the stellar spectra from the reduced datacube. The locations at which to extract the flux are not fully determined by PAMPelMuse, instead, it uses the catalogue of the ACS Survey as an initial guess (after correcting for a global offset). In its newest version, modified for the use with NFM data, PAMPELMusE then searches for the star in a small 2D region around the catalogue position. For each star, the offsets $\delta x, \delta y$ relative to the catalogue position are recorded for later use. The offsets together with the observation dates form a time-series $\left(t_{i}, \delta x_{i}\right)$. Ignoring any effects due to binarity or calibration, the relation between $t_{i}$ and $\delta x_{i}$ should be linear, i.e. $\delta_{x}=v_{x} t+x_{0}$, where $v_{x}$ is the proper motion in the $x$ direction. Computationally, $v_{x}$ and $v_{y}$ are determined by fitting a straight line to $t_{i}$ and $\delta x_{i}$ and $t_{i}$ and $\delta y_{i}$, respectively.

We apply the described method to M80 where we have two NFM observations, taken on May 4, 2019, and February 24, 2020. Together with the 


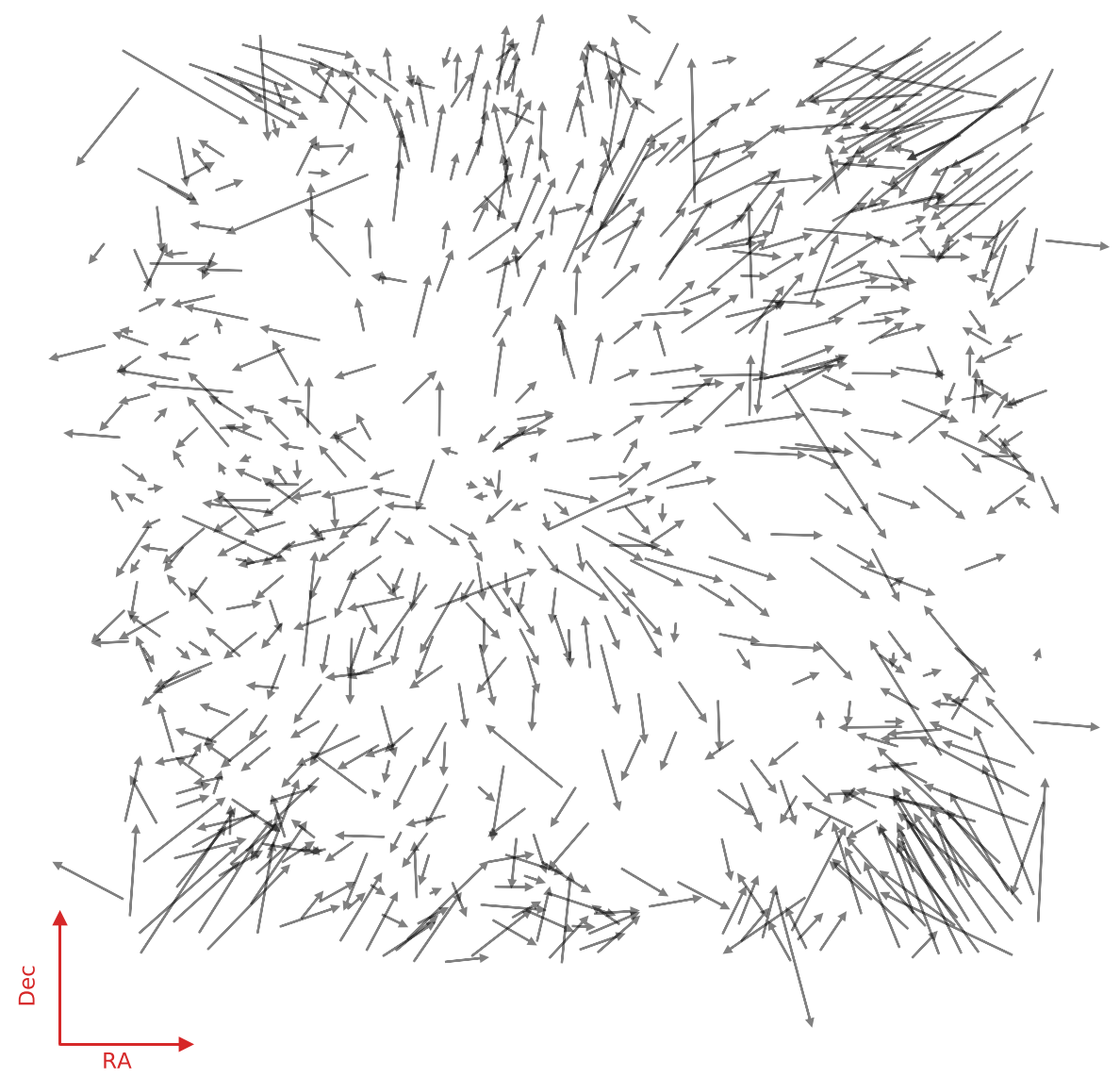

Figure 4.1: Proper-motions in the central $7.5 \times 7.5 \operatorname{arcsec}^{2}$ (the MUSE NFM field-of-view) of M80. Each arrow starts at a ACS catalogue position of a star. The $\mathrm{x}$ - and $\mathrm{y}$-component of each arrow is proportional to the proper motion in that direction. 
Table 4.1: Percentiles of the distributions of proper motions $v_{x}, v_{y}$, and radial velocity $v_{r}$, as well as their statistical uncertainties. All values are given in $\mathrm{km} / \mathrm{s}$.

\begin{tabular}{lrrr}
\hline & \multicolumn{3}{c}{ percentile } \\
parameter & 16th & 50 th & 84th \\
\hline$v_{x}$ & -27.5 & 1.6 & 30.0 \\
$v_{y}$ & -22.7 & 2.0 & 30.2 \\
$v_{r}$ & -12.8 & -0.4 & 12.0 \\
$v_{x, \text { unc. }}$ & 3.2 & 11.9 & 26.0 \\
$v_{y, \text { unc. }}$ & 2.9 & 10.3 & 23.6 \\
$v_{r, \text { unc. }}$ & 3.3 & 5.9 & 9.9 \\
\hline
\end{tabular}

corresponding HST observation used for the ACS catalogue of M80 taken on April 6, 2006 (Anderson et al. 2008), the baseline is about 14 years. The total number of stars in each MUSE NFM observation that we can extract with PAMPELMUSE is about 1000. However, about 700 stars that are in the ACS catalogue are extracted from both NFM datacubes.

Figure 4.1 shows the measured proper motions as arrows. Each arrow starts at a catalogue position of a star. The $\mathrm{x}$ - and y-component of each arrow is $v_{x}$ and $v_{y}$, respectively. In all four corners of the MUSE NFM field-of-view, there is a large number of correlated motions towards the centre. This is very likely an artefact, possibly caused by optical distortion. The proper motions seem to be correlated nearly everywhere in the plot, i.e. stars close to each other have moved in a similar direction with a similar speed. This too seems to be caused by instrumental effects, e.g. uncorrected small-scale distortions that would similarly affect neighbouring stars.

Table 4.1 lists percentiles for the distributions of velocity components and their uncertainties. The order of magnitude of the velocities is the same as the radial velocity. However, the standard deviation is about $30 \mathrm{~km} / \mathrm{s}$ and roughly twice as large as the standard deviation of the radial velocities. This property cannot only be explained by the larger uncertainties. It is thus very likely that systematic effects are dominant here. This is not totally unexpected as MUSE is not designed to be an astrometric instrument.

A possible way to quantify distortions and other systematic effects would be a direct comparison of proper motions of individual stars measured with high astrometric quality (e.g. HSTPROMO) and the offsets determined in the way described above for the same stars. This should be possible for NGC 5139 where proper motions measured from HST are available (Bellini et al. 2017a) and NFM observations also exist. 


\subsection{Future observations with MUSE and BlueMUSE}

While the MUSE wide-field mode (WFM) has been used to observe Galactic and extra-galactic GCs with and without adaptive optics for several years now, the possibility to observe with the NFM is relatively new and more restricting in terms of environmental conditions and the choice of a guide star. With recent relaxations to the guide star choice (ESO, 2021) and thus an increase in the possible locations in the sky where it can be used, the NFM might be used more frequently in the future. In the case of GC observations, this could mean more freedom in the exact positioning in a GC core or even the possibility of off-centre observations of interesting objects that are too strongly affected by crowding or are too faint for the WFM. Taking into account the high throughput of the NFM and the very large number of stars that can be simultaneously observed (about 1000 stars in case of our observations of M80), repeated observations of GC cores can efficiently detect binaries similar to Giesers et al. (2019). Future repeated NFM observations of central GC fields will allow the detection of binaries in the GC core for stars that are not even visible in WFM observations due to crowding or brightness. Because of mass segregation, the heaviest binaries, and potentially triple systems, are expected closer to the GC centre than less heavy systems. Besides interesting binary systems such as those with a stellar-mass black holes, neutron stars, or white dwarfs, companion stars of any potential IMBH could also be found this way (see Section 4.3). The potential to observe the brightest white dwarfs of a GC with the NFM is currently under investigation.

BlueMUSE is a proposed integral-field spectrograph for the VLT optimised for observations in the blue spectral region (Richard et al. 2019). Compared to MUSE, it is planned to have a field of view twice as large, a higher average spectral resolution of $R \approx 3600$ but a lower spatial sampling of 0.3 arcsec and no $\mathrm{AO}$ system. The planned spectral range extends from 350 to $600 \mathrm{~nm}$ compared to 480 to $930 \mathrm{~nm}$ for MUSE. For observations of globular clusters, BlueMUSE will allow the observation of spectral lines bluewards of $\mathrm{H} \beta$, in particular the $\mathrm{H}$ and $\mathrm{K}$ lines caused by calcium. The larger density of spectral lines, mostly from iron, in this region compared to the region available with MUSE will decrease the uncertainty of the metallicity determined with a full-spectrum fit. It will also improve measurements of the radial velocity which is expected to lead to more sensitive searches for radial velocity variations to detect binaries. Kinematical properties of the whole cluster, in particular the rotation and velocity dispersion profiles, can be measured more accurately. These analyses also benefit from the larger field of view which captures stars at a larger central distance and thus increases the spatial coverage compared to a single observation with MUSE. 
The spectroscopic analysis of multiple populations (see Section 1.2.6) will be possible with BlueMUSE since the $\mathrm{CN}$ molecular band is in its spectral range. Instead of the more usual separation of stars into populations by using pseudocolours derived from a few photometric filters, BlueMUSE will allow separation by spectroscopic properties (e.g. Hollyhead et al. 2017). Not only red-giant branch stars will be accessible this way, but also main-sequence stars which are less evolved and perhaps offer clues to the origin of multiple populations. 


\section{BibLIOGRAPHY}

Aarseth S. J., 1999, Publications of the Astronomical Society of the Pacific, 111, 1333

Anderson J., et al., 2008, The Astronomical Journal, 135, 2055

Aros F. I., Sippel A. C., Mastrobuono-Battisti A., Askar A., Bianchini P., van de Ven G., 2020, Monthly Notices of the Royal Astronomical Society, 499, 4646

Bachetti M., et al., 2014, Nature, 514, 202

Bacon R., Monnet G., 2017, Optical 3D-Spectroscopy for Astronomy. John Wiley \& Sons

Bacon R., et al., 2010. San Diego, California, USA, p. 773508, doi:10.1117/12.856027, http://proceedings.spiedigitallibrary.org/ proceeding. aspx?doi=10.1117/12.856027

Bajkova A. T., Bobylev V. V., 2020, arXiv e-prints, 2008, arXiv:2008.13624

Bastian N., Lardo C., 2018, Annual Review of Astronomy and Astrophysics, 56, 83

Baumgardt H., 2017, Monthly Notices of the Royal Astronomical Society, 464, 2174

Baumgardt H., Hilker M., 2018, Monthly Notices of the Royal Astronomical Society, 478, 1520

Baumgardt H., Makino J., Hut P., McMillan S., Portegies Zwart S., 2003, The Astrophysical Journal Letters, 589, L25

Baumgardt H., Makino J., Hut P., 2005, The Astrophysical Journal, 620, 238

Baumgardt H., Hilker M., Sollima A., Bellini A., 2019a, Monthly Notices of the Royal Astronomical Society. 482, 5138 
Baumgardt H., et al., 2019b, Monthly Notices of the Royal Astronomical Society, 488,5340

Bekki K., Freeman K. C., 2003, Monthly Notices of the Royal Astronomical Society. 346, L11

Bellini A., Anderson J., Bedin L. R., King I. R., van der Marel R. P., Piotto G., Cool A., 2017a, The Astrophysical Journal, 842, 6

Bellini A., Bianchini P., Varri A. L., Anderson J., Piotto G., van der Marel R. P., Vesperini E., Watkins L. L., 2017b, The Astrophysical Journal, 844, 167

Belloni D., Giersz M., Rivera Sandoval L. E., Askar A., Ciecieląg P., 2019, Monthly Notices of the Royal Astronomical Society. 483, 315

Benacquista M. J., Downing J. M. B., 2013, Living Reviews in Relativity, 16, 4

Bianchini P., van der Marel R. P., del Pino A., Watkins L. L., Bellini A., Fardal M. A., Libralato M., Sills A., 2018, Monthly Notices of the Royal Astronomical Society, 481,2125

Binney J., Tremaine S., 2008, Galactic Dynamics: Second Edition. https://ui. adsabs.harvard.edu/abs/2008gady. book..... B

Bond H. E., 2015, The Astronomical Journal, 149, 132

Brightman M., Walton D. J., Xu Y., Earnshaw H. P., Harrison F. A., Stern D., Barret D., 2020, The Astrophysical Journal, 889, 71

Brok M. d., et al., 2015, The Astrophysical Journal, 809, 101

Busoni L., Agapito G., Plantet C., Oberti S., Verinaud C., Le Louarn M., Esposito S., Ciliegi P., 2020, arXiv e-prints, p. arXiv:2012.14626

Cappellari M., 2008, Monthly Notices of the Royal Astronomical Society. 390, 71

Carretta E., Bragaglia A., Gratton R., Lucatello S., 2009, Astronomy \& Astrophysics, 505, 139

Cen R., 2001, The Astrophysical Journal, 560, 592

Chomiuk L., Strader J., Maccarone T. J., Miller-Jones J. C. A., Heinke C., Noyola E., Seth A. C., Ransom S., 2013, The Astrophysical Journal. 777, 69

Contenta F., Gieles M., Balbinot E., Collins M. L. M., 2017, Monthly Notices of the Royal Astronomical Society, 466, 1741 
Cordoni G., Milone A. P., Mastrobuono-Battisti A., Marino A. F., Lagioia E. P., Tailo M., Baumgardt H., Hilker M., 2020a, The Astrophysical Journal, 889, 18

Cordoni G., et al., 2020b, The Astrophysical Journal. 898, 147

Davies R., Kasper M., 2012, Annual Review of Astronomy and Astrophysics 50,305

Davis T. A., et al., 2020, Monthly Notices of the Royal Astronomical Society. 496,4061

ESO 2013, The globular star cluster 47 Tucanae, https : //www . eso . org/public/ images/eso1302a/

ESO 2015, The MUSE instrument on the VLT: equipped with 24 continuous flow cooling systems, https://www . eso.org/public/images/ann15041a/

ESO 2021, MUSE News, http://web.archive.org/web/20210617080619/ https://wWw.eso.org/sci/facilities/paranal/instruments/muse/ news.html

Emsellem E., Monnet G., Bacon R., 1994, Astronomy and Astrophysics, 285, 723

Feast M. W., Thackeray A. D., 1960, Monthly Notices of the Royal Astronomical Society, 120, 463

Forbes D. A., 2020, Monthly Notices of the Royal Astronomical Society. 493, 847

Forbes D. A., et al., 2018, Proceedings of the Royal Society A: Mathematical, Physical and Engineering Sciences, 474, 20170616

Fétick R. J. L., et al., 2019, Astronomy and Astrophysics, 628, A99

Gardner J. P., et al., 2006, Space Science Reviews. 123, 485

Gebhardt K., Pryor C., O'Connell R. D., Williams T. B., Hesser J. E., 2000, The Astronomical Journal, 119, 1268

Gebhardt K., Rich R. M., Ho L. C., 2002, The Astrophysical Journal Letters, 578, L41

Gebhardt K., Rich R. M., Ho L. C., 2005, The Astrophysical Journal, 634, 1093

Genzel R., Eisenhauer F., Gillessen S., 2010, Reviews of Modern Physics, 82, 3121

Ghez A. M., et al., 2008, The Astrophysical Journal, 689, 1044 
Gieles M., Zocchi A., 2015, Monthly Notices of the Royal Astronomical Society, 454,576

Gieles M., Balbinot E., Yaaqib R. I. S. M., Hénault-Brunet V., Zocchi A., Peuten M., Jonker P. G., 2018, Monthly Notices of the Royal Astronomical Society. 473,4832

Giersz M., 1998, Monthly Notices of the Royal Astronomical Society, 298, 1239

Giersz M., Leigh N., Hypki A., Lützgendorf N., Askar A., 2015, Monthly Notices of the Royal Astronomical Society, 454, 3150

Giesers B., et al., 2018, Monthly Notices of the Royal Astronomical Society. 475, L15

Giesers B., et al., 2019, Astronomy \& Astrophysics, 632, A3

Gill M., Trenti M., Miller M. C., Marel R. v. d., Hamilton D., Stiavelli M., 2008, The Astrophysical Journal, 686, 303

Gillett F. C., Jacoby G. H., Joyce R. R., Cohen J. G., Neugebauer G., Soifer B. T., Nakajima T., Matthews K., 1989, The Astrophysical Journal, 338, 862

Gilmozzi R., Spyromilio J., 2007, The Messenger, 127, 11

González E., Kremer K., Chatterjee S., Fragione G., Rodriguez C. L., Weatherford N. C., Ye C. S., Rasio F. A., 2021, The Astrophysical Journal Letters, 908, L29

Graham-Smith F., 2016, Eyes on the Sky: A Spectrum of Telescopes. Oxford University Press, Oxford, New York

Greene J. E., Strader J., Ho L. C., 2020, Annual Review of Astronomy and Astrophysics, 58, 257

Gunn J. E., Griffin R. F., 1979, The Astronomical Journal, 84, 752

Göttgens F., 2017, Master's thesis, Georg-August-Universität Göttingen, Göttingen

Göttgens F., et al., 2019a, A\&A, 626, A69

Göttgens F., et al., 2019b, A\&A, 631, A118

Göttgens F., et al., 2021, Monthly Notices of the Royal Astronomical Society. 507,4788

Haffert S. Y., Bohn A. J., de Boer J., Snellen I. A. G., Brinchmann J., Girard J. H., Keller C. U., Bacon R., 2019, Nature Astronomy, 3, 749 
Hameury J. M., 2020, Advances in Space Research 66, 1004

Hameury J.-M., Lasota J.-P., 2020, Astronomy \& Astrophysics, 643, A171

Harris W. E., 1996, The Astronomical Journal, 112, 1487

Hearnshaw J., 2010, Journal of Astronomical History and Heritage, 13, 90

Hearnshaw J. B., 2014, The Analysis of Starlight: Two Centuries of Astronomical Spectroscopy. Cambridge University Press

Hogg H. S., Wehlau A., 1964, The Astronomical Journal, 69, 141

Hollyhead K., et al., 2017, Monthly Notices of the Royal Astronomical Society: Letters, 465, L39

Husser T.-O., Wende-von Berg S., Dreizler S., Homeier D., Reiners A., Barman T., Hauschildt P. H., 2013, Astronomy \& Astrophysics, 553, A6

Hénault-Brunet V., Gieles M., Agertz O., Read J. I., 2015, Monthly Notices of the Royal Astronomical Society. 450, 1164

Hénault-Brunet V., Gieles M., Sollima A., Watkins L. L., Zocchi A., Claydon I., Pancino E., Baumgardt H., 2019, Monthly Notices of the Royal Astronomical Society, 483, 1400

Hénault-Brunet V., Gieles M., Strader J., Peuten M., Balbinot E., Douglas K. E. K., 2020, Monthly Notices of the Royal Astronomical Society, 491, 113

Jacoby G. H., Morse J. A., Fullton L. K., Kwitter K. B., Henry R. B. C., 1997, The Astronomical Journal, 114, 2611

Jacoby G. H., Ciardullo R., Marco O. D., Lee M. G., Herrmann K. A., Hwang H. S., Kaplan E., Davies J. E., 2013, The Astrophysical Journal, 769, 10

Jacoby G. H., Marco O. D., Davies J., Lotarevich I., Bond H. E., Harrington J. P., Lanz T., 2017, The Astrophysical Journal, 836, 93

Jalali B., Baumgardt H., Kissler-Patig M., Gebhardt K., Noyola E., Lützgendorf N., Zeeuw P. T. d., 2012, Astronomy \& Astrophysics, 538, A19

Joshi K. J., Rasio F. A., Zwart S. P., 2000, The Astrophysical Journal, 540, 969

Kamann S., Wisotzki L., Roth M. M., 2013, Astronomy and Astrophysics, 549, A71

Kamann S., et al., 2018, Monthly Notices of the Royal Astronomical Society. 473,5591 
Kamann S., et al., 2020a, Monthly Notices of the Royal Astronomical Society, 492,966

Kamann S., et al., 2020b, Monthly Notices of the Royal Astronomical Society. 492,2177

Kamann S., et al., 2020c, Astronomy \&amp; Astrophysics, 635, A65

Knigge C., 2012, Mem.Soc.Ast.It., 83, 549

Kollatschny W., Weilbacher P. M., Ochmann M. W., Chelouche D., MonrealIbero A., Bacon R., Contini T., 2020, Astronomy \& Astrophysics, 633, A79

Kong A. K. H., 2007, The Astrophysical Journal, 661, 875

Kremer K., Rui N. Z., Weatherford N. C., Chatterjee S., Fragione G., Rasio F. A., Rodriguez C. L., Ye C. S., 2021, arXiv e-prints, 2104, arXiv:2104.11751

Kruijssen J. M. D., 2015, Monthly Notices of the Royal Astronomical Society. 454,1658

Kruijssen J. M. D., Pfeffer J. L., Reina-Campos M., Crain R. A., Bastian N., 2019, Monthly Notices of the Royal Astronomical Society. 486, 3180

Kruijssen J. M. D., et al., 2020, Monthly Notices of the Royal Astronomical Society, 498,2472

Kizıltan B., Baumgardt H., Loeb A., 2017, Nature, 542, 203

LIGO Scientific Collaboration and Virgo Collaboration et al., 2020, Physical Review Letters, 125, 101102

Latour M., et al., 2019, Astronomy and Astrophysics, 631, A14

Lilly S., 1998, The Next Generation Space Telescope (NGST): Submission to the Long Range Planning Panel, http://www . casca.ca/lrp/vol2/ngst/hstng. html

Lützgendorf N., Kissler-Patig M., Noyola E., Jalali B., de Zeeuw P. T., Gebhardt K., Baumgardt H., 2011, Astronomy and Astrophysics, 533, A36

MacLeod M., Trenti M., Ramirez-Ruiz E., 2016, The Astrophysical Journal, 819, 70

Maccarone T. J., 2004, Monthly Notices of the Royal Astronomical Society, 351, 1049

Magorrian J., et al., 1998, The Astronomical Journal, 115, 2285 
Mann C. R., et al., 2019, The Astrophysical Journal. 875, 1

Martocchia S., et al., 2018a, Monthly Notices of the Royal Astronomical Society. 473,2688

Martocchia S., et al., 2018b, Monthly Notices of the Royal Astronomical Society. 477,4696

Martocchia S., et al., 2020, Monthly Notices of the Royal Astronomical Society, 499,1200

Massari D., Koppelman H. H., Helmi A., 2019, Astronomy \& Astrophysics, 630, L4

Mayor M., et al., 2003, The Messenger, 114, 20

Meylan G., Sarajedini A., Jablonka P., Djorgovski S. G., Bridges T., Rich R. M., 2001, The Astronomical Journal, 122, 830

Mezcua M., 2017, International Journal of Modern Physics D. 26, 1730021

Miller-Jones J. C. A., et al., 2012, The Astrophysical Journal Letters, 755, L1

Monty S., et al., 2021, arXiv e-prints, p. arXiv:2107.13199

Neumayer N., Seth A., Böker T., 2020, Astronomy and Astrophysics Review, 28, 4

Nitadori K., Aarseth S. J., 2012, Monthly Notices of the Royal Astronomical Society, 424, 545

Noyola E., Gebhardt K., Bergmann M., 2008, The Astrophysical Journal, 676, 1008

Pease F. G., 1928, Publications of the Astronomical Society of the Pacific 40, 342

Pepe F., et al., 2014, Astronomische Nachrichten, 335, 8

Perera B. B. P., et al., 2017, Monthly Notices of the Royal Astronomical Society, 468,2114

Piotto G., et al., 2015, The Astronomical Journal, 149, 91

Pooley D., Rappaport S., 2006, The Astrophysical Journal Letters, 644, L45

Portegies Zwart S. F., McMillan S. L. W., 2002, The Astrophysical Journal, 576, 899

Richard J., et al., 2019, arXiv:1906.01657 [astro-ph] 
Rigaut F., Neichel B., 2018, Annual Review of Astronomy and Astrophysics, 56, 277

Rizzuto F. P., et al., 2021, Monthly Notices of the Royal Astronomical Society. 501,5257

Rui N. Z., Weatherford N. C., Kremer K., Chatterjee S., Fragione G., Rasio F. A., Rodriguez C. L., Ye C. S., 2021, Research Notes of the AAS, 5, 47

Ryu J., Lee M. G., 2018, The Astrophysical Journal Letters, 863, L38

Sahman D. I., Dhillon V. S., Knigge C., Marsh T. R., 2015, Monthly Notices of the Royal Astronomical Society, 451, 2863

Sahman D. I., Dhillon V. S., Littlefair S. P., Hallinan G., 2018, Monthly Notices of the Royal Astronomical Society, 477, 4483

Salaris M., Cassisi S., 2005, Evolution of stars and stellar populations. J. Wiley, Chichester, West Sussex, England ; Hoboken, NJ, USA

Saracino S., et al., 2020, Monthly Notices of the Royal Astronomical Society, 498,4472

Sarajedini A., et al., 2007, The Astronomical Journal, 133, 1658

Sawyer H. B., 1938, Journal of the Royal Astronomical Society of Canada, 32, 69

Schwarzschild M., 1979, The Astrophysical Journal, 232, 236

Shara M. M., et al., 2007, Nature, 446, 159

Sollima A., Baumgardt H., Hilker M., 2019, Monthly Notices of the Royal Astronomical Society, 485, 1460

Steinicke W., 2017, Observing and cataloguing nebulae and star clusters: from Herschel to Dreyer's New General Catalogue. Cambridge University Press, Cambridge

Stoyan R., Binnewies S., Friedrich S., 2008, Atlas of the Messier Objects: Highlights of the Deep Sky. Cambridge University Press, Cambridge, doi:10.1017/CBO9780511536502 https: //www.cambridge.org/core/books/atlas-of-the-messier-objects/ A45098338EC4DCDDB0D84386CECFCF1A

Strader J., Chomiuk L., Maccarone T. J., Miller-Jones J. C. A., Seth A. C., 2012, Nature, 490, 71 
Ströbele S., et al., 2012, in Adaptive Optics Systems III. International Society for Optics and Photonics, p. 844737, doi:10.1117/12.926110. https://wwW.spiedigitallibrary.org/conference-proceedings-ofspie/8447/844737/GALACSI-system-design-and-analysis/10.1117/12. 926110.short

Stuik R., Bacon R., Conzelmann R., Delabre B., Fedrigo E., Hubin N., Le Louarn M., Ströbele S., 2006, New Astronomy Reviews, 49, 618

Tremou E., et al., 2018, The Astrophysical Journal, 862, 16

Trenti M., Padoan P., Jimenez R., 2015, The Astrophysical Journal, 808, L35

Tyson R. K., 2011, Principles of Adaptive Optics. CRC Press

Ulvestad J. S., Greene J. E., Ho L. C., 2007, The Astrophysical Journal Letters, 661, L151

Valcin D., Bernal J. L., Jimenez R., Verde L., Wandelt B. D., 2020, Journal of Cosmology and Astroparticle Physics, 2020, 002

VandenBerg D. A., Brogaard K., Leaman R., Casagrande L., 2013, The Astrophysical Journal 775,134

Varri A. L., Bertin G., 2012, Astronomy and Astrophysics, 540, A94

Vasiliev E., Baumgardt H., 2021, arXiv:2102.09568 [astro-ph]

Vitral E., Mamon G. A., 2021, Astronomy \& Astrophysics, 646, A63

Voggel K., Hilker M., Baumgardt H., Collins M. L. M., Grebel E. K., Husemann B., Richtler T., Frank M. J., 2016, Monthly Notices of the Royal Astronomical Society, 460, 3384

Warner B., 1995, in Cambridge Astrophysics, Cataclysmic Variable Stars. Cambridge University Press, pp 126-215, doi:10.1017/CBO9780511586491.004

Watkins L. L., van de Ven G., den Brok M., van den Bosch R. C. E., 2013, Monthly Notices of the Royal Astronomical Society, 436, 2598

Watkins L. L., van der Marel R. P., Bellini A., Anderson J., 2015, The Astrophysical Journal, 803, 29

Webb J. J., Carlberg R. G., 2021, Monthly Notices of the Royal Astronomical Society, 502, 4547 
Wilson R. N., 1999, Reflecting Telescope Optics II: Manufacture, Testing, Alignment, Modern Techniques. Astronomy and Astrophysics Library, Astronomy,Astrophysics Library Wilson,R.:Reflecting Telescope Opt., SpringerVerlag, Berlin Heidelberg, doi:10.1007/978-3-662-08488-5, https://www. springer.com/de/book/9783540603566

Wilson O. C., Coffeen M. F., 1954, The Astrophysical Journal, 119, 197

Woo J.-H., Cho H., Gallo E., Hodges-Kluck E., Le H. A. N., Shin J., Son D., Horst J. C., 2019, Nature Astronomy, 3, 755

Xie C., Haffert S. Y., de Boer J., Kenworthy M. A., Brinchmann J., Girard J., Snellen I. a. G., Keller C. U., 2020, Astronomy \&amp; Astrophysics. 644, A149

Yeh F.-C., Carraro G., Korchagin V. I., Pianta C., Ortolani S., 2020, Astronomy \& Astrophysics, 635, A125

Zocchi A., Gieles M., Hénault-Brunet V., 2017, Monthly Notices of the Royal Astronomical Society 468, 4429

de Vita R., Trenti M., Bianchini P., Askar A., Giersz M., van de Ven G., 2017, Monthly Notices of the Royal Astronomical Society, 467, 4057

van der Marel R. P., Anderson J., 2010, The Astrophysical Journal, 710, 1063 


\section{Appendix A}

\section{Appendix}

\section{A.1 A Stellar Census in Globular Clusters with MUSE: A Spectral Catalogue of Emission-Line Sources (Göttgens et al., 2019b)}

The following publication consists of material published in my master's thesis (Göttgens, 2017) with updates and additions as described in Chapter 3

Reproduced with permission from Astronomy \& Astrophysics, @ ESO 


\title{
A stellar census in globular clusters with MUSE: A spectral catalogue of emission-line sources ${ }^{\star}$
}

\author{
Fabian Göttgens ${ }^{1}$, Tim-Oliver Husser ${ }^{1}$, Sebastian Kamann ${ }^{2}$, Stefan Dreizler ${ }^{1}$, Benjamin Giesers ${ }^{1}$, \\ Wolfram Kollatschny ${ }^{1}$, Peter M. Weilbacher ${ }^{3}$, Martin M. Roth ${ }^{3}$, and Martin Wendt ${ }^{4}$ \\ ${ }^{1}$ Institut für Astrophysik, Georg-August-Universität Göttingen, Friedrich-Hund-Platz 1, 37077 Göttingen, Germany \\ e-mail: fabian.goettgens@uni-goettingen.de \\ 2 Astrophysics Research Institute, Liverpool John Moores University, 146 Brownlow Hill, Liverpool L3 5RF, UK \\ 3 Leibniz-Institut für Astrophysik Potsdam (AIP), An der Sternwarte 16, 14482 Potsdam, Germany \\ ${ }^{4}$ Institut für Physik und Astronomie, Universität Potsdam, Karl-Liebknecht-Str. 24/25, 14476 Golm, Germany \\ Received 9 August 2019 / Accepted 10 September 2019
}

\begin{abstract}
Aims. Globular clusters produce many exotic stars due to a much higher frequency of dynamical interactions in their dense stellar environments. Some of these objects were observed together with several hundred thousand other stars in our MUSE survey of 26 Galactic globular clusters. Assuming that at least a few exotic stars have exotic spectra (i.e. spectra that contain emission lines), we can use this large spectroscopic data set of over a million stellar spectra as a blind survey to detect stellar exotica in globular clusters. Methods. To detect emission lines in each spectrum, we modelled the expected shape of an emission line as a Gaussian curve. This template was used for matched filtering on the differences between each observed 1D spectrum and its fitted spectral model. The spectra with the most significant detections of $\mathrm{H} \alpha$ emission are checked visually and cross-matched with published catalogues.

Results. We find 156 stars with $\mathrm{H} \alpha$ emission, including several known cataclysmic variables (CV) and two new CVs, pulsating variable stars, eclipsing binary stars, the optical counterpart of a known black hole, several probable sub-subgiants and red stragglers, and 21 background emission-line galaxies. We find possible optical counterparts to $39 \mathrm{X}$-ray sources, as we detected $\mathrm{H} \alpha$ emission in several spectra of stars that are close to known positions of Chandra X-ray sources. This spectral catalogue can be used to supplement existing or future X-ray or radio observations with spectra of potential optical counterparts to classify the sources.
\end{abstract}

Key words. globular clusters: general - stars: emission-line, Be - novae, cataclysmic variables - catalogs techniques: imaging spectroscopy

\section{Introduction}

In the dense stellar environments of globular clusters (GCs), the frequent interactions between stars produce a wealth of stellar exotica. This includes interacting binary systems and end states of stellar evolution, such as cataclysmic variables (CVs, Ivanova et al. 2006), pulsars (Ransom 2007), and planetary nebulae (PNe; Jacoby et al. 2017). Emission lines are expected to appear in the optical spectra in at least some of these stellar types; those stars are then classified as emission-line stars. Because of the old age of globular clusters and their stars, some types of emission-line stars still present in the Milky Way disc do not exist (anymore) in globular clusters, for example Wolf-Rayet stars or Be stars.

In recent years, several stellar-mass black hole $(\mathrm{BH})$ candidates have been found in binary systems in globular clusters (Strader et al. 2012; Giesers et al. 2018). Photometric observations suggest that some of these systems could be $\mathrm{H} \alpha$ emitters. While stellar-mass BHs were long thought to be ejected from GCs during cluster evolution, the discoveries of stellarmass $\mathrm{BH}$ candidates in multiple clusters indicate a large population of these black holes inside evolved GCs (Strader et al. 2012; Askar et al. 2018; Kremer et al. 2018).

* Table A.2 and spectra (FITS) are available at the CDS via anonymous ftp to cdsarc.u-strasbg.fr $(130.79 .128 .5)$ or via http: //cdsarc.u-strasbg.fr/viz-bin/cat/J/A+A/631/A118
Cataclysmic variables are binary systems consisting of a hot, compact white dwarf and a dwarf star in a close orbit. The white dwarf accretes material from its companion star that accumulates in an accretion disc. In the dense stellar environments of globular clusters, CVs and progenitor systems are influenced by dynamical interactions, with up to $50 \%$ forming via a binary encounter (Ivanova et al. 2006, but also see Belloni et al. 2019). The number of predicted CVs per cluster is of the order of 200 , but the number of observed CV candidates or confirmed CVs in the literature is much lower (Knigge 2012). CV candidates can be found with photometric observations, for example by looking for dwarf nova outbursts, for stars with UV excess (e.g. Rivera Sandoval et al. 2018), for outliers in the colourmagnitude diagram (CMD) of a GC (e.g. Campos et al. 2018), or by using $\mathrm{H} \alpha$ surveys (Knigge 2012). Alternatives for detecting CVs are far-ultraviolet spectroscopy which has also been useful for detecting CVs in globular clusters (Knigge et al. 2003), and $\mathrm{X}$-ray observations. Follow-up optical spectroscopy to confirm $\mathrm{CVs}$ in GCs is difficult because of the crowded fields and the intrinsically low brightness of CVs.

When a nova occurs in a CV, it can leave behind a visible emission nebula as a remnant such as the one in NGC 6656 (Göttgens et al. 2019). Nova remnants are not the only type of nebula in GCs; another type are planetary nebulae of which four are known in the Galactic GC system. Even this low number of $\mathrm{PNe}$ in GCs is too high because the low masses of AGB stars should prohibit the formation of PNe (Jacoby et al. 1997). This 
lead to the prediction that PNe in GCs are formed by a different mechanism, possibly by binary interaction (Jacoby et al. 1997, 2017).

Many stellar exotica in GCs have been found using X-ray observations. However, it is less clear which optical counterpart belongs to an X-ray source when only broad-band photometry is available. In this case, a counterpart is identified if it is an outlier in the optical CMD with respect to all other cluster stars, that is if its separation from the main sequence or the red-giant branch (RGB) is too large, or if its colour is too blue (e.g. Bassa et al. 2004; Webb et al. 2004). Similarly, the presence of optical emission lines in a spectrum of a star close to an X-ray source could also indicate it is a counterpart.

Previous optical surveys for typical classes of emissionline objects used photometric observations and the on/off-band technique, variability, or anomalous colours to detect candidate objects: Jacoby et al. (1997) conducted the most successful PNe survey for GCs, Knigge (2012) lists several CV surveys. Spectroscopic follow-up observations are then used to confirm the classification and to derive more properties of the source.

The data used in this paper were obtained with the Multi Unit Spectroscopic Explorer (MUSE, Bacon et al. 2010), a panoramic integral-field spectrograph at the Very Large Telescope, as part of a survey of Galactic globular clusters. With the MUSE data already obtained, emission-line objects can be found without the need of additional observations because both spatial and spectral information is present. While Roth et al. (2018) demonstrated the efficiency of MUSE at detecting emission-line objects including Wolf-Rayet stars, supernova remnants, H II regions, and PNe in the galaxy NGC 300, we can for the first time conduct a blind survey for emission-line stars in Galactic globular clusters.

\section{Data}

\subsection{Observations and reduction}

This work makes use of all data taken with MUSE for our survey of 26 Galactic globular clusters between September 2014 and March 2019 (PI: S. Dreizler, S. Kamann) ${ }^{1}$. MUSE has a large field of view $\left(1^{\prime} \times 1^{\prime}\right)$ combined with a spatial sampling of $0.2^{\prime \prime}$ and an intermediate resolution $R$ between 1800 and 3500 in the spectral range from 4750 to $9350 \AA$. The observations and the analysis steps are described in detail by Kamann et al. (2018) and are summarised here. In contrast to Kamann et al. (2018), this work also includes data from observations made after October 2016. Table 1 gives an overview of the observation statistics for each cluster.

Each observation was reduced with the standard MUSE pipeline (Weilbacher et al. 2012, 2014) which calibrates the images from the 24 MUSE spectrographs, including cosmic ray rejection, and transforms them into a datacube. In the next step, single stellar spectra are extracted with a point-spread-function (PSF) from this datacube. The extractions use the PSF-fitting developed in Kamann et al. (2013) to measure the PSF parameters and determine stellar positions in the datacube as a function of wavelength. We mostly used stellar positions from the Hubble Space Telescope (HST) ACS survey of Galactic globular clusters (hereafter ACS catalogue, Sarajedini et al. 2007; Anderson et al. 2008) as an input for the extraction, see Table 2 in Kamann et al. (2018) for details. The extracted spectra were then analysed with

1 ESO Programme IDs: 094.D-0142, 095.D-0629, 096.D-0175, 097.D-0295, 098.D-0148, 099.D-0019, 0100.D-0161, 0101.D-0268, and 0102.D-0270.
Table 1. Overview of globular cluster data used in this paper.

\begin{tabular}{|c|c|c|c|c|c|c|}
\hline $\begin{array}{l}\text { NGC } \\
(1)\end{array}$ & $\begin{array}{c}\text { Name } \\
(2)\end{array}$ & $\begin{array}{r}N_{\text {pointings }} \\
\text { (3) }\end{array}$ & $\begin{array}{r}N_{\text {epochs }} \\
(4)\end{array}$ & $\begin{array}{r}\text { ToT [h] } \\
\text { (5) }\end{array}$ & $\begin{array}{r}N_{\text {spectra }} \\
(6)\end{array}$ & $\begin{array}{r}N_{\text {stars }} \\
(7)\end{array}$ \\
\hline 104 & 47 Tuc & 10 & 13 & 12.2 & 309911 & 32055 \\
\hline 362 & & 6 & 2 & 1.3 & 24049 & 9363 \\
\hline 1851 & & 4 & 6.5 & 4.7 & 67267 & 11614 \\
\hline 1904 & M 79 & 4 & 5.5 & 2.5 & 32597 & 5669 \\
\hline 2808 & & 4 & 2 & 1.2 & 20230 & 8040 \\
\hline 3201 & & 5 & 12 & 11.6 & 61855 & 4503 \\
\hline 5139 & $\omega$ Cen & 10 & 10.5 & 12.2 & 335614 & 45616 \\
\hline 5286 & & 1 & 4 & 1.0 & 17954 & 8282 \\
\hline 5904 & M 5 & 6 & 2 & 2.3 & 51450 & 18203 \\
\hline 6093 & M 80 & 4 & 2 & 1.6 & 21051 & 9153 \\
\hline 6121 & M 4 & 2 & 1 & 0.1 & 1251 & 1067 \\
\hline 6218 & & 4 & 3 & 3.0 & 22989 & 6616 \\
\hline 6254 & M 10 & 8 & 1.5 & 3.3 & 29633 & 14296 \\
\hline 6266 & M 62 & 4 & 3 & 2.0 & 39190 & 15900 \\
\hline 6293 & & 1 & 2 & 0.1 & 2154 & 1326 \\
\hline 6388 & & 4 & 4 & 2.2 & 46600 & 14484 \\
\hline 6441 & & 4 & 4 & 2.8 & 43473 & 13247 \\
\hline 6522 & & 1 & 3 & 0.2 & 7564 & 3567 \\
\hline 6541 & & 5 & 2 & 1.9 & 35352 & 12003 \\
\hline 6624 & & 1 & 2 & 0.5 & 8300 & 4556 \\
\hline 6656 & M 22 & 4 & 2.5 & 2.2 & 36609 & 13204 \\
\hline 6681 & M 70 & 1 & 2 & 0.8 & 8283 & 4773 \\
\hline 6752 & & 8 & 2 & 3.0 & 31070 & 14086 \\
\hline 7078 & M 15 & 4 & 3 & 1.6 & 40606 & 18015 \\
\hline 7089 & M 2 & 4 & 4 & 2.4 & 47764 & 15309 \\
\hline 7099 & M 30 & 4 & 3.5 & 2.6 & 34176 & 9111 \\
\hline Total & & 114 & 103.0 & 80.8 & 1379362 & 316428 \\
\hline
\end{tabular}

Notes. This includes all observations made between September 2014 and March 2019. (1) NGC number. (2) Alternative identifier (if any). (3) Number of pointings. This number roughly corresponds to the covered field of view in arcminutes. (4) Average number of epochs available for each pointing. (5) Total integration time in hours. (6) Number of extracted spectra. (7) Number of stars with at least one extracted spectrum.

respect to the Göttingen spectral library (Husser et al. 2013), a grid of synthetic spectral models suitable for most stars in globular clusters. A chi-square fit on the full spectrum minimises the difference between the observed spectrum and a model spectrum by interpolating between grid spectra to determine the stellar parameters effective temperature, metallicity, and the radial velocity (Husser et al. 2016).

In addition to the spectra obtained from single observations, we also used these to create a high-signal-to-noise spectrum for each star. We shifted all spectra of each star to the Sun's restframe and added the flux weighted by the signal-to-noise $(\mathrm{S} / \mathrm{N})$ ratio of the spectrum. These combined spectra were then analysed in a similar way to the one described above.

\subsection{Residuals from spectral fitting}

The residuals from the spectral fitting are defined as the difference between model and observation. We can use these residuals to detect emission-line stars because the spectral library does not contain spectra with emission lines; in other words, if an emission line is present in the observed spectrum, it will also be visible in the residuals.

The residuals can contain random noise, additional absorption from the interstellar medium (Wendt et al. 2017), systematic 


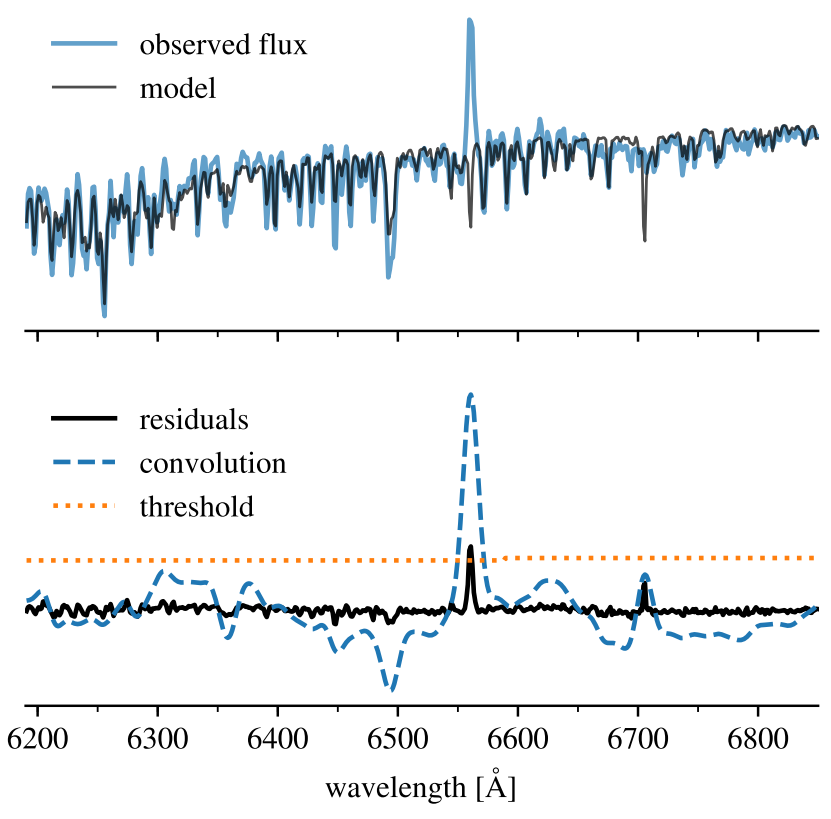

Fig. 1. Illustration of emission-line detection with matched filtering. Top: observed flux of V190 in NGC 6266 with $\mathrm{H} \alpha$ emission and the fitted spectral model. Bottom: $\mathrm{H} \alpha$ emission line is still present in the residuals, as well as an absorption line at $6700 \AA$ which is only found in the models. The convolution with a Gaussian increases the emission line above the threshold calculated from the mean absolute deviation of the residuals.

errors of the model spectra (e.g. absorption lines that only exist in the models, see Fig. 1, or vice versa), instrumental systematics, and true emission lines. If the fit does not find the global minimum of the chi-square space, the residuals will contain a large amount of stellar light. In this case, the parameters determined by the fit do not necessarily describe the star, and the residuals can cause false positive detections of emission lines.

It is also possible that the spectral model grid does not contain a suitable model for the observed spectrum. This occurs for horizontal branch stars and some M stars. Spectra of M stars contain strong molecular bands which have a great influence on the overall spectral appearance. A slight mismatch in the fit of an $\mathbf{M}$ star spectrum has a large impact on the residuals. If a spectrum of an $\mathrm{M}$ star contains emission lines, the effect of the emission lines on the residuals could be smaller than the effects of spectrum mismatch. In these cases, the method based on matched filtering to detect emission lines described in Sect. 3.1 was not reliable and the detection failed. However, the method based on the residuals without convolution (Sect. 3.2) still worked in these cases.

\section{Emission line detection}

As shown in Table 1, we extracted millions of stellar spectra from our observations. The large number of observed spectra makes it impossible to visually check each of them.

We used two approaches to detect emission lines in the residuals from the spectral fit. The first approach based on matched filtering is widely applied to similar problems, for example in gravitational-wave detection (Abbott et al. 2016) and to detect emission-line galaxies in MUSE datacubes (Herenz \& Wisotzki 2017). The second method uses only the residuals and a running estimate of the noise. This method is used as a backup whenever matched filtering fails to detect a signal, because it is much simpler and more robust but also produces more false detections. Both approaches assign a significance to each detection which is then used to select the most promising candidates for visual inspection.

We stress that we did not use existing catalogues of emissionline stars as a prior to find those in our data. Since the aim of this work is to find new and unexpected sources, we used external catalogues only after our methods identify a possible spectrum with emission lines.

\subsection{Matched filtering with mean absolute deviation}

Because of the large dataset, we needed to choose an approach that is fast and can extract potentially weak signals. One algorithm with these properties is called matched filtering (see Vio \& Andreani 2016, and references therein) that requires prior knowledge about the expected signal. We assumed that each emission line can be described by a Gaussian curve with a standard deviation (width) of $5 \AA$. This width is determined from simulations in Sect. 3.3. Instead of applying matched filtering directly on the observed spectral flux (top panel in Fig. 1), we used it on the residuals that result from spectral fitting (see bottom panel of Fig. 1). Mathematically, matched filtering computes the convolution $C(\lambda)$ of the filter (the expected line profile) and the residual flux. As shown in the bottom panel in Fig. 1 (dashed line), the convolution is high at a certain wavelength when the expected flux shape matches the measured flux. On the other hand, noise - which is typically only a few pixels wide - is smoothed out, that means the convolution gives a much lower value as it would for real emission. Compared to the convolution of noise or continuum flux with a Gaussian, emission flux appears in the convolution as a peak centred at the emission line. We detect an emission signal at wavelength $\lambda_{\mathrm{e}}$ if the convolution at that point is larger than some threshold function $t(\lambda)$ at the same point. The threshold function is constructed as the median absolute deviation calculated separately for wavelength bins of the residual flux (dotted line in the bottom panel of Fig. 1). By construction, the ratio $D_{\mathrm{s}}=C\left(\lambda_{\mathrm{e}}\right) / t\left(\lambda_{\mathrm{e}}\right)$ is higher for more prominent emission signals. We call this ratio detection significance and use it to select promising candidates for visual inspection by requiring that a detection lies above a minimum value of $D_{\mathrm{s}}$. The detection efficiency depends on this choice and it is analysed with simulated emission lines in Sect. 3.3.

\subsection{Plain residuals and running noise estimate}

This section presents a more robust method of detecting emission lines that relies on fewer assumptions. Similar to the method based on matched filtering, it relies on the residuals from the spectral fit. The residual flux $r_{\mathrm{i}}=r\left(\lambda_{\mathrm{i}}\right)$ at each wavelength point $\lambda_{\mathrm{i}}$ is compared to the residual noise $s_{\mathrm{i}}$ at the adjacent wavelengths. We locally estimated the noise from the difference of the ninetieth and tenth percentile of the residual flux in a $100 \AA$ window centred at $\lambda_{\mathrm{i}}$. Since the spectral model does not describe the observed flux perfectly, the residuals contain noise and systematic effects (see Sect. 2.2). We accounted for these outliers in the residual flux by using percentiles instead of extrema or measures that are sensitive to outliers. At each wavelength, the ratio of residual flux to the noise estimate $D_{\mathrm{s}}=r_{\mathrm{i}} / s_{\mathrm{i}}$ represents the significance of an emission-line detection. For comparison, if the noise was normally distributed with a variance $\sigma^{2}$, a ratio of $D_{\mathrm{s}}=1$ corresponds to an observation with a significance of $\approx 1.3 \sigma$.

Simulations showed that this method works well for narrow emission lines but not for broad ones. This is because a 


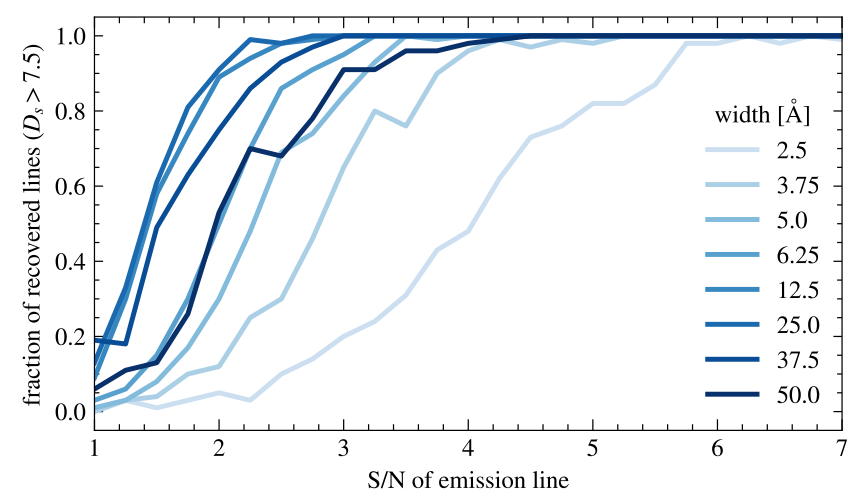

Fig. 2. Fraction of simulated emission lines that are recovered with a filter width of $6 \AA$ Aepending on the simulated line width. Only detections above a detection significance $D_{\mathrm{s}}>7.5$ are taken into account.

broad emission line increases the residuals over a broader spectral range, and thus the percentiles used to estimate the noise increase as well. Since this increases $s_{\mathrm{i}}$ but not the amplitude $r_{\mathrm{i}}$, the detection significance $D_{\mathrm{s}}$ decreases accordingly.

\subsection{Detection efficiency}

We estimated the fraction of emission signals recovered with the method based on matched filtering described in Sect. 3.1 with simulated emission lines. The detection significance of an emission-line candidate depends on the amplitude and width of the emission peak in the residual flux, and on the noise of the spectrum and the width of the Gaussian filter. We constructed emission lines by sampling a Gaussian curve with a standard deviation $\sigma$ that we vary between 3 and $60 \AA$, its amplitude is set to one. We drew noise from a normal distribution with a width of $(\mathrm{S} / \mathrm{N})^{-1}$ and add it to the signal. For each simulated emission line, we applied the detection method (Sect. 3.1) and calculated the detection significance for filter widths of $6,25,60$, and $120 \AA$. We note that this leads to an estimate of how effective the detection methods is with respect to the amplitude of an emission line and not the total line flux.

The main results of this analysis were: we can find broad synthetic emission lines of several tens of Angström even with a narrow filter width of $6 \AA$ while the reverse is not true. As Fig. 2 shows, we recovered about $50 \%$ of broad emission lines $(\approx 40 \AA)$ with a filter width of $6 \AA$ for a S/N of 2 if we only take detections with $D_{\mathrm{s}}>7.5$ into account. This fraction increased with increasing $\mathrm{S} / \mathrm{N}$ and with decreasing emission-line width (except for very narrow widths $<5 \AA$ below the filter width). We concluded from these simulated emission lines that for our data the choice of a $6 \AA$ filter is reasonable, and $D_{\mathrm{s}}>7.5$ is a good lower limit for a detection to be inspected further. The filter width is four to five times the FWHM of the line-spread-function (LSF) of MUSE which varies between 2.5 and $3 \AA$ depending on wavelength (Bacon et al. 2017). In principle, one could choose the threshold $D_{\mathrm{s}}$ much lower than this for the price of many more detections to inspect, which will contain a much higher frequency of false positives. The choice of $D_{\mathrm{s}}>7.5$ is also justified by the low empirical true positive rate of $\lesssim 5 \%$ below this limit (see Sect. 4.1 and Fig. 4).

\subsection{Limiting flux}

The results of the simulations above were used to calculate the minimum amplitude of an emission line that can be detected.
Table 2. Limiting fluxes for all clusters, calculated using a broad emission line $(40 \AA)$ ) and also valid for narrow lines (see text).

\begin{tabular}{|c|c|c|c|}
\hline \multirow[t]{2}{*}{ Cluster } & \multicolumn{3}{|c|}{$\log _{10} F_{\min }\left[\operatorname{erg~s}^{-1} \mathrm{~cm}^{-2} \AA^{-1}\right]$} \\
\hline & MS & TO & RGB \\
\hline NGC 104 & $-16.9_{-0.5}^{+0.3}$ & $-16.7_{-0.5}^{+0.3}$ & $-16.3_{-0.2}^{+0.4}$ \\
\hline NGC 1851 & $-17.5_{-0.2}^{+0.3}$ & $-17.3_{-0.3}^{+0.3}$ & $-17.0_{-0.3}^{+0.5}$ \\
\hline NGC 1904 & $-17.5_{-0.2}^{+0.3}$ & $-17.4_{-0.2}^{+0.3}$ & $-16.9_{-0.2}^{+0.4}$ \\
\hline NGC 2808 & $-17.4_{-0.2}^{+0.3}$ & $-17.3_{-0.2}^{+0.3}$ & $-16.8_{-0.2}^{+0.4}$ \\
\hline NGC 3201 & $-17.6_{-0.2}^{+0.3}$ & $-17.4_{-0.1}^{+0.3}$ & $-16.6_{-0.1}^{+0.1}$ \\
\hline NGC 362 & $-17.1_{-0.2}^{+0.3}$ & $-17.1_{-0.2}^{+0.3}$ & $-16.5_{-0.3}^{+0.5}$ \\
\hline NGC 5139 & $-17.1_{-0.2}^{+0.3}$ & $-16.9_{-0.2}^{+0.3}$ & $-16.5_{-0.2}^{+0.2}$ \\
\hline NGC 5286 & $-17.6_{-0.2}^{+0.3}$ & $-17.5_{-0.2}^{+0.3}$ & $-17.2_{-0.2}^{+0.4}$ \\
\hline NGC 5904 & $-17.4_{-0.3}^{+0.3}$ & $-17.2_{-0.3}^{+0.3}$ & $-16.6_{-0.1}^{+0.4}$ \\
\hline NGC 6093 & $-17.5_{-0.2}^{+0.3}$ & $-17.5_{-0.2}^{+0.3}$ & $-17.1_{-0.4}^{+0.1}$ \\
\hline NGC 6121 & $-16.9_{-0.1}^{+0.3}$ & $-16.9_{-0.1}^{+0.2}$ & $-16.4_{-0.0}^{+0.0}$ \\
\hline NGC 6218 & $-17.8_{-0.1}^{+0.3}$ & $-17.4_{-0.1}^{+0.2}$ & $-16.9_{-0.1}^{+0.3}$ \\
\hline NGC 6254 & $-17.6_{-0.3}^{+0.3}$ & $-17.4_{-0.2}^{+0.3}$ & $-16.7_{-0.1}^{+0.3}$ \\
\hline NGC 6266 & $-17.6_{-0.2}^{+0.3}$ & $-17.4_{-0.2}^{+0.4}$ & $-16.9_{-0.5}^{+0.1}$ \\
\hline NGC 6293 & $-17.1_{-0.2}^{+0.2}$ & $-17.2_{-0.2}^{+0.2}$ & $-16.8_{-0.2}^{+0.4}$ \\
\hline NGC 6388 & $-17.5_{-0.2}^{+0.2}$ & $-17.4_{-0.3}^{+0.2}$ & $-17.1_{-0.4}^{+0.2}$ \\
\hline NGC 6441 & $-17.6_{-0.1}^{+0.2}$ & $-17.5_{-0.2}^{+0.2}$ & $-17.2_{-0.3}^{+0.2}$ \\
\hline NGC 6522 & $-17.4_{-01}^{+0.3}$ & $-17.4_{-01}^{+0.3}$ & $-17.0_{-02}^{+0.3}$ \\
\hline NGC 6541 & $-17.4_{-0.2}^{+0.3}$ & $-17.3_{-0.2}^{+0.3}$ & $-16.9_{-0.3}^{+0.2}$ \\
\hline NGC 6624 & $-17.6_{-0.2}^{+0.3}$ & $-17.6_{-0.2}^{+0.3}$ & $-17.2_{-0.5}^{+0.1}$ \\
\hline NGC 6656 & $-17.2_{-0.3}^{+0.2}$ & $-17.1_{-0.3}^{+0.4}$ & $-16.5_{-0.1}^{+0.2}$ \\
\hline NGC 6681 & $-17.8_{-0.2}^{+0.3}$ & $-17.7_{-0.2}^{+0.3}$ & $-17.2_{-0.1}^{+0.1}$ \\
\hline NGC 6752 & $-17.3_{-0.4}^{+0.2}$ & $-17.0_{-0.4}^{+0.2}$ & $-16.6_{-0.3}^{+0.3}$ \\
\hline NGC 7078 & $-17.5_{-0.2}^{+0.4}$ & $-17.4_{-0.3}^{+0.4}$ & $-16.8_{-0.3}^{+0.5}$ \\
\hline NGC 7089 & $-17.4_{-0.2}^{+0.2}$ & $-17.3_{-0.2}^{+0.3}$ & $-16.9_{-0.2}^{+0.3}$ \\
\hline NGC 7099 & $-17.6_{-0.2}^{+0.2}$ & $-17.4_{-0.2}^{+0.2}$ & $-16.9_{-0.2}^{+0.2}$ \\
\hline
\end{tabular}

Notes. Uncertainties give the central $80 \%$ of the distribution on the main-sequence (MS), main-sequence turn-off (TO), and red giant branch (RGB).

Since we selected detections with a significance above 7.5, emission lines in spectra with too high noise will not be found. From the simulations, we first estimated the minimum signal-to-noise ratio $S / N_{\min }$ for that $80 \%$ of simulated emission lines are found. This $S / N_{\text {min }}$ depends on the width of the simulated emission line. Here, we chose a width of $40 \AA$, corresponding to CVs. The minimum signal that we could detect is estimated by measuring the noise $\sigma$ in the residuals of all spectra from $6000 \AA$ to $7000 \AA$. In practice, the noise depends on the brightness of the target star, observing conditions, stellar crowding, etc. We measured this effective noise in the residuals obtained from the spectral fitting. The minimum detectable signal in each spectrum is $F_{\text {min }}=S / N_{\text {min }} \cdot \sigma$. Table 2 lists $F_{\text {min }}$ of a broad emission line for different representative points in the stellar population of each cluster we observed, and Fig. 3 shows the limiting flux as a function of stellar brightness in three clusters. Since we used a $S / N_{\min }$ for which $80 \%$ of simulated emission lines are found, Table 2 gives the limiting flux for which $80 \%$ of all spectra with an emission line are found. Because we used a narrow filter width of $5 \AA$ to detect emission lines, the limits given for 


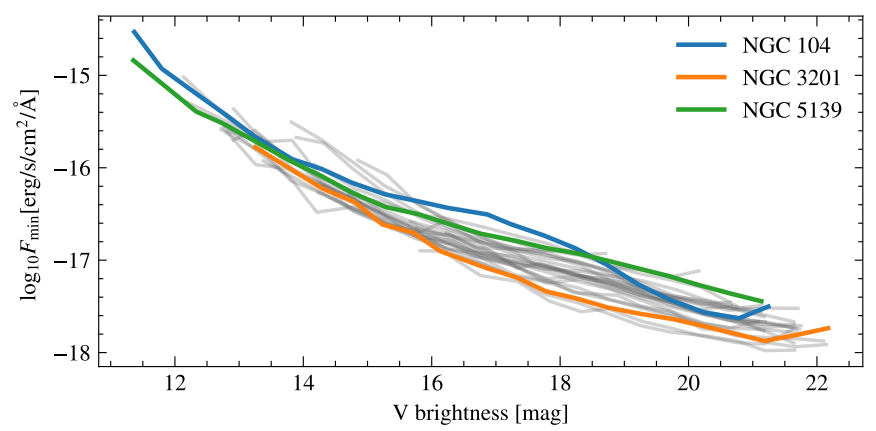

Fig. 3. Limiting flux for which $80 \%$ of sources will be detected as determined from simulated emission lines for different clusters as a function of brightness. Each grey curve represents a GC, the clusters NGC 104, NGC 3201, and NGC 5139 are highlighted.

a broad emission line can be treated as a conservative estimate of the limiting flux of narrow emission lines. The limiting fluxes for narrow emission lines generally fall inside the uncertainties given in Table 2, this means that this table is also valid for narrow lines. Depending on the brightness of the target star, we find that $F_{\min }$ is generally between $10^{-17}$ and $10^{-16} \mathrm{erg} \mathrm{s}^{-1} \mathrm{~cm}^{-2} \AA^{-1}$. To our knowledge, this is the first optical emission-line survey estimating the upper limit of fluxes for sources that remain undetected.

\section{The catalogue of emission-line sources}

\subsection{Results of visual inspection}

We applied both detection methods to spectra extracted from MUSE observations of globular clusters (see Sect. 2.1) that have an $\mathrm{S} / \mathrm{N}$ of at least five. Setting this threshold ensures that the spectral fit gives meaningful residuals. We wanted to inspect promising emission-line spectra visually. As this would have been very time consuming with thousands of candidate spectra, we checked candidate spectra which contain an emission line close to $\mathrm{H} \alpha$ (between 6540 and $6580 \AA$ ) if $D_{\mathrm{s}}>7.5$. With this set-up, we expect to find emission-line stars, typically $\mathrm{H} \alpha$ emitters, while galaxies would remain undetected. Section 4.7 describes how we find galaxies using all detected emission lines. In total, 1200 individual stars have at least one such spectrum, with a total of about 9000 spectra.

For each spectrum, we checked if the emission line could be valid according to a set of criteria. The potential emission line has to be at least two pixels wide (a pixel corresponds to $1.25 \AA$ ) and it must fulfil at least one of the following criteria:

- The line candidate appears in roughly the same position with the same shape in multiple spectra of the same star, or

- the spectrum shows emission lines in addition to $\mathrm{H} \alpha$, or

- the corresponding star is listed in the Catalogue of Variable Stars in Galactic Globular Clusters (CVSGGC, Clement et al. 2001; Clement 2017), Simbad (Wenger et al. 2000), or in a suitable catalogue in Vizier (Ochsenbein et al. 2000), or

- the star is close to an X-ray source as listed in the Chandra Source Catalog Release 2.0 (Evans et al. 2010).

Typically, an emission-line candidate is not valid if the spectrum seems to be contaminated by other stars or nebulae. This occured if a much brighter star is close $\left(\approx 2^{\prime \prime}\right.$ or less $)$ to the target star, or if it was close to one of the three nebulae in our survey.

Inspection of the results show that false positives are mainly caused by noise, contamination by brighter stars, and poor fit

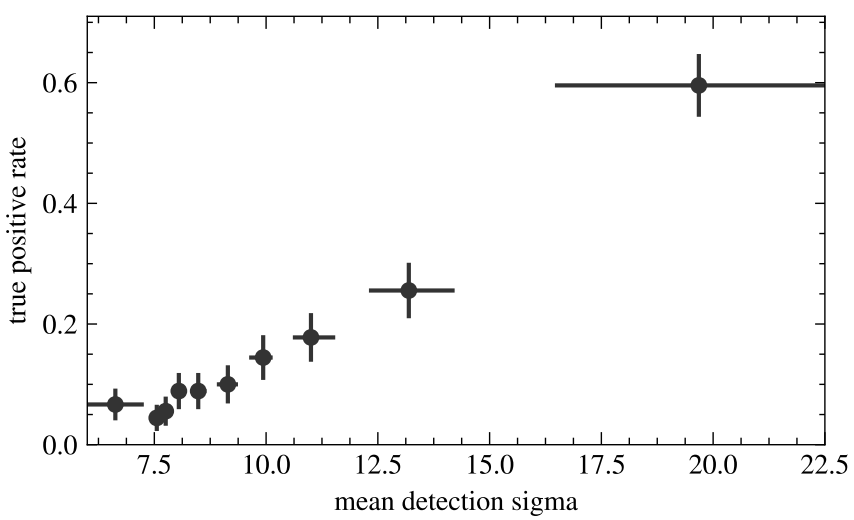

Fig. 4. Empirical true positive rate of matched filtering after checking about 1200 stars with detected emission lines. Each bin contains 90 stars and the errorbars in $x$-direction contain the central $1 \sigma$ interval of the detection significance per bin.

results. Figure 4 shows the empirical true positive rate after a visual check of each star with $D_{\mathrm{s}}>7.5$. For testing purposes, we also checked emission-line candidates with a lower significance than 7.5, these stars are also included in this figure. As expected, the true positive rate correlates with the mean detection significance and reaches about $60 \%$ for $D_{\mathrm{s}}>6$.

Table A.2 lists all stars with spectra containing valid emission lines that we found in our survey. This table also gives the original ID used in the ACS catalogue in column "ACS ID". The columns " $d_{\mathrm{C}}$ " lists the projected distance to the respective cluster centre. The table also contains our estimate whether the star is a likely cluster member in column "mem.?". In contrast to Kamann et al. (2018), this estimate is based on radial velocities only. Column "vrad?" contains an indicator whether the star shows variations in its radial velocity based on the method described in Giesers et al. (2019). We converted the probability of variability calculated in Giesers et al. (2019) in the following way: $p<0.15$ : not variable, $p>0.85$ : variable, $0.15<p<0.86$ : unsure (?). Blank fields indicate insufficient data. We expect a false positive rate of $15 \%$. Cross-matches with other catalogues and papers are given in column "Ident." with the corresponding reference in column "Ref.". The column " $d_{\mathrm{X}}$ " contains the separation to the next Chandra X-ray source (Evans et al. 2010), if it is less than the positional uncertainties of the X-ray source. Background galaxies are listed in Table 3.

\subsection{Cataclysmic variables}

As described above, CVs are binary systems consisting of a hot, compact white dwarf and a dwarf star in a close orbit. Only ten CVs have been confirmed by spectroscopy in the whole globular cluster system of the Milky Way (Knigge 2012; Webb \& Servillat 2013). Most CV candidates identified by photometry are not bright enough to be observed with MUSE and our relatively short exposure times. For example, Rivera Sandoval et al. (2018) lists $R_{625}$ magnitudes for $21 \mathrm{CV}$ candidates in NGC 104 of which only four are brighter than $20 \mathrm{mag}$.

\subsubsection{Known CVs and confirming CV candidates}

We find nine CVs, of which seven are either previously spectroscopically confirmed CVs or candidates. The normalised MUSE spectra of several CVs including the previously unknown ones are shown in Fig. 5. Characteristic broad Balmer emission lines 


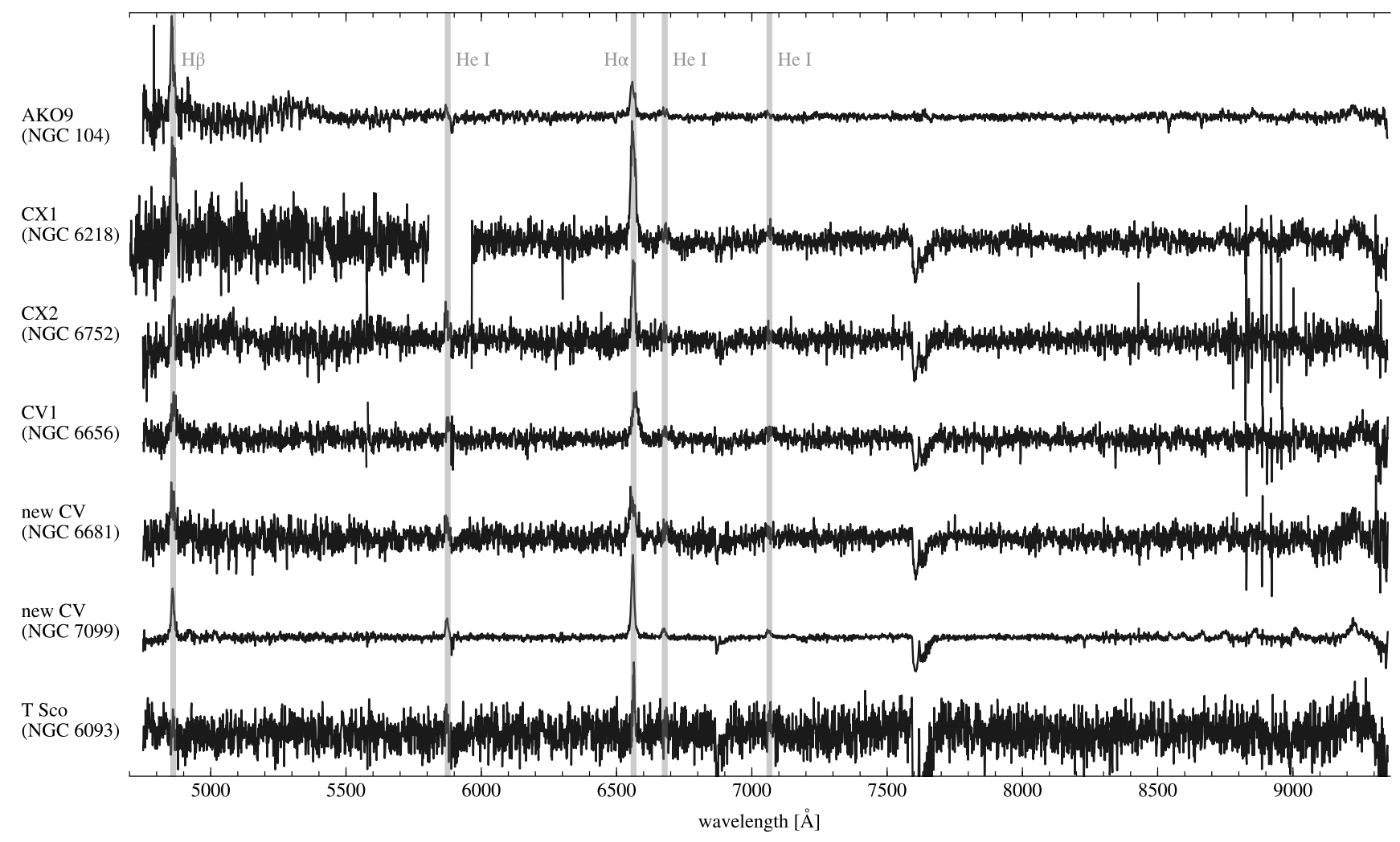

Fig. 5. Normalised spectra of known cataclysmic variables AKO9, CX1, CX2, CV1, of the new CVs in NGC 6681 and NGC 7099, and of the CV underlying the Nova T Sco in 1860. All spectra are detected by their broad Balmer emission lines, they also show He I emission. The spectrum shown for AKO9 was created by combining several observed spectra.

of $\mathrm{H} \alpha$ and $\mathrm{H} \beta$ are clearly visible in all spectra, as well as $\mathrm{He} \mathrm{I}$ emission.

One of them, CX1 in NGC6218 was detected as an $\mathrm{X}$-ray source with optical counterpart and classified as a CV by Lu et al. (2009) who consider it to be a member of the cluster based on its X-ray luminosity. We find that its optical counterpart is not the star marked in their finding chart but rather the star directly to the east with a F606W magnitude of 20.8. With this new counterpart, we can confirm that $\mathrm{CX} 1$ is indeed a $\mathrm{CV}$.

We do not see the characteristic broad Balmer emission lines for a CV in any spectrum of W56/X6 in NGC 104 which was classified as a CV in Heinke et al. (2005). However, the spectra show a $\mathrm{H} \alpha$ absorption line that is less deep than our spectral model predicts. Since this is not clearly a CV, we do not include it in our discussion in Sect. 5.1.

\subsubsection{Nova T Scorpii}

In 1860, a classical nova in NGC 6093 was observed by Pogson (1860), Nova T Scorpii. Both Shara \& Drissen (1995) and Dieball et al. (2010) looked for the underlying CV using near- and far-UV observations and they found a UV bright source at the right spatial position. Using the finding charts in Dieball et al. (2010), we can identify their source 2129 with ACS ID 44184 (F336W-F438W =-0.1, F438W = 18.5, Piotto et al. 2015; Soto et al. 2017). This star was independently detected by our algorithm because of its broad $\mathrm{H} \alpha$ emission in several of its ten spectra observed with MUSE. A visual inspection shows that also $\mathrm{H} \beta$ and a weak He I emission are present and variable. The $\mathrm{H} \alpha$ and $\mathrm{H} \beta$ lines seem to switch between emission and absorption. However, as ACS ID 44184 is located on the lower RGB in the optical CMD, the CV has either a giant donor star or it is not resolved in the HST photometry but instead blended with a unrelated star.

\subsubsection{New cataclysmic variables}

Additionally to the seven known CVs, we find two more stars with very similar emission lines, indicating that these two stars are CVs as well. One new CV is close to the centre of NGC 7099 with a distance of $11^{\prime \prime}$ and a F606W magnitude of 20.3 in the ACS catalogue (ACS ID 23423). Based on its position close to an X-ray source and its blue $U-V$ colour, Lugger et al. (2007) identified this star as a possible $\mathrm{CV}$ candidate (source $\mathrm{C}$ ). However, it is not included as a CV in the CVSGGC, which is why we list it as a new CV here.

The new CV in NGC 6681 (ACS ID 19706) has a distance of 27 " to the cluster centre and a F606W magnitude of 22.7. The spectra of the new and known CVs are shown in Fig. 5. Both new CVs are close $\left(0.13\right.$ and $0.25^{\prime \prime}$, respectively) to a Chandra X-ray source listed in the November 2017 pre-release of the Chandra Source Catalog Release 2.0 (Evans et al. 2010). Although NGC 6681 was observed with HST in the UV (see e.g. Massari et al. 2013) and with the Chandra X-ray observatory (Pooley 2007; Dieball 2008), no articles about CVs in this cluster have been published.

Are these CVs actually part of their respective cluster? In general, we use the radial velocity and metallicity to determine if a star is a member of a globular cluster or a field star. The standard spectral fit failed to determine reliable radial velocities or metallicities from the spectra of the new CVs. We used a Gaussian fit to the $\mathrm{H} \alpha$ line to estimate the radial velocity for each $\mathrm{CV}$, including the known $\mathrm{CV}$ s. The velocities differ from 
the cluster values by up to $300 \mathrm{~km} \mathrm{~s}^{-1}$. This could be because the emission lines in some cases seem to have a more complex, that is non-Gaussian, shape, or because of intrinsically high velocity variations due to the orbital motions, or possibly because of eclipses of the accretion disc as observed for AKO9 (Knigge et al. 2003).

We assume that CVs in GCs are spatially distributed in the same way as all other stars in a GC. In the simulations of Belloni et al. (2019) CVs are either distributed more centrally or in the same way as main-sequence stars, depending on the relation time of the GCs. With this property of CVs we can also use Bayes factors to decide between the two hypotheses $A \equiv$ "CV is a cluster member" and $B \equiv$ " $\mathrm{CV}$ is not a cluster member". To calculate the factors we made use of the spatial distribution and membership probability derived from the observed radial velocity and metallicity of all the other stars observed with MUSE in the same field of view (see Kamann et al. 2018 for details). The distances of the new CVs to the respective cluster centre are about $1 / 6$ of the half-light radius (NGC 7099) and 2/3 (NGC 6681) when the values from Harris (1996) are used. In the MUSE field of view (FoV) of NGC $709996 \%$ of all stars are cluster members; this leaves about $4 \%$ non-members. Of all member stars, $8 \%$ are closer to the cluster centre than the $\mathrm{CV}$ we consider, while the remaining $92 \%$ are farther away. Thus, the likelihood of being a member star and at the same separation from the cluster centre or even closer is $p_{A}=0.074$. As for non-members, $10 \%$ lie closer to the centre than the $\mathrm{CV}$, and $90 \%$ are farther away. This gives $p_{B}=0.004$. The Bayes factor is $p_{A} / p_{B} \approx 18$, which means that the positions of the CV provide evidence in favour of hypothesis $A$. The same analysis for the $\mathrm{CV}$ that may be associated with NGC 6681 gives a Bayes factor of $p_{A} / p_{B} \approx 13$. Here, $92 \%$ are member stars, of which two third lie closer to the centre than the CV. Of the $8 \%$ non-members, $55 \%$ lie closer to the centre. According to the interpretation of Jeffreys (1998, p. 432), Bayes factors between 10 and $10^{3 / 2}$ provide strong evidence in favour of hypothesis $A$. In conclusion, the positions of the CVs and all the other stars in the MUSE FoV strongly suggest that both CVs are members of the respective cluster.

\subsection{Optical counterpart of M62-VLA1}

Several stellar mass black holes or candidates are known in globular clusters, including three in NGC 3201 (Giesers et al. 2018, 2019), M22-VLA1 and -VLA2 in NGC 6656 (Strader et al. 2012), and M62-VLA1 in NGC 6266 (Chomiuk et al. 2013). The black-hole candidates in NGC 3201 were discovered by Giesers et al. $(2018,2019)$ using variations in the radial velocities of their visible companions observed with MUSE. These discoveries demonstrate that MUSE observations can be used to detect stellar exotica in GCs.

The black-hole candidate M62-VLA1 is close to the centre of NGC 6266 and was discovered by Strader et al. (2012) using radio and X-ray observations. It is likely to be part of a binary system with a star on the lower RGB, which the authors identified in HST images very close to their radio source. Our emission-line search found the optical counterpart of this black hole because of its $\mathrm{H} \alpha$ emission line. Both the position of this star and the counterpart reported in Strader et al. (2012) match, as well as the location in the colour-magnitude diagram. This star was observed several times with MUSE in 2015 and again in 2018 with varying S/N. The spectra with the highest S/N show a $\mathrm{H} \alpha$ emission line which seem to vary between observations. These variations in the $\mathrm{H} \alpha$ region are shown in Fig. 6 where

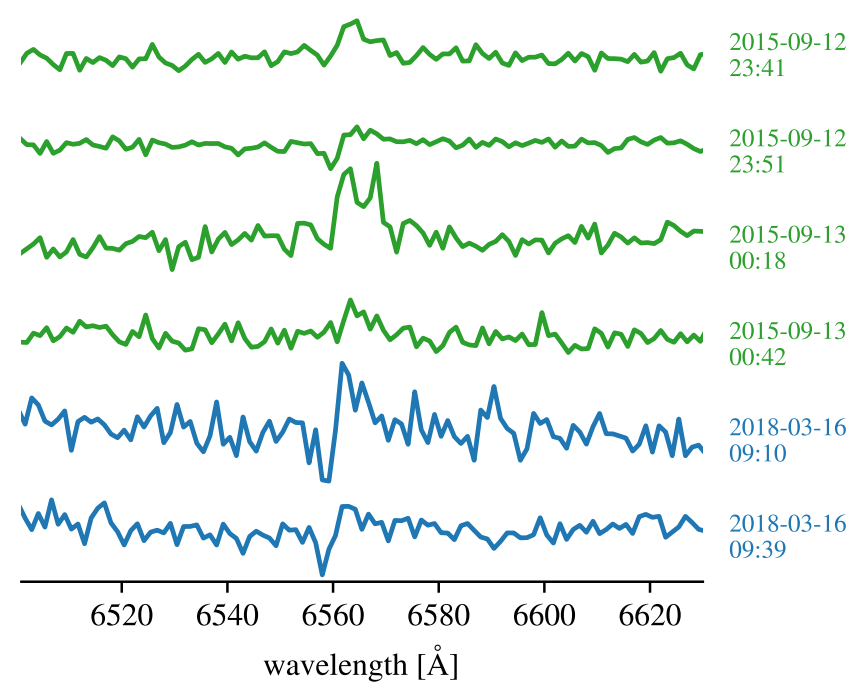

Fig. 6. $\mathrm{H} \alpha$ region of multiple spectra of the likely companion of the black-hole candidate M62-VLA1. This part of the spectrum is variable on the timescale of minutes.

the shape of the emission line changes within tens of minutes. We need more observations to determine reliable orbital parameters for this system similarly to the black holes and the 92 other binary systems in NGC 3201 (Giesers et al. 2019).

\subsection{Red stragglers and sub-subgiants}

Red stragglers (RS) and sub-subgiants (SSG) are stars in globular clusters that occupy the region redwards of the RGB or below the subgiant branch in the CMD. Since stellar evolution theory predicts these regions to be empty, their existence needs to be explained by more complicated formation theories (Geller et al. 2017a,b; Leiner et al. 2017). In addition to their unusual location in the CMD, $\mathrm{RS}$ are X-ray and $\mathrm{H} \alpha$ emitters, photometrically variable and mostly radial-velocity binaries (Geller et al. 2017a). As expected, several detected emission-line stars fall into the CMD region occupied by RS (four stars) and SSG (12 stars, see column "ID" in Table A.2). In particular, we detected the RS binary in NGC 6254 discovered by Shishkovsky et al. (2018) which is also a source of radio and X-ray radiation. A similar case is a star (ACS ID 40733) in the RS region of NGC 6541 which has a very broad and variable $\mathrm{H} \alpha$ emission, and it is close to an X-ray source $\left(0^{\prime \prime}, 18\right)$. Of the eleven SSG that show $\mathrm{H} \alpha$ emission and are probable cluster members, eight are close to an X-ray source, eight show variations in their radial velocities, and seven SSG are both close to an X-ray source and have radial velocity variations. All four RS with $\mathrm{H} \alpha$ emission are members of their respective cluster, three are close to an X-ray source and those three RS also show radial velocity variations. We did not detect variability in the radial velocities of the fourth RS and it is not associated with an X-ray source. These correlations fit the general characteristics of SSG and RS as described in Geller et al. (2017a). Orbital parameters for several SSG systems in NGC 3201, including the $\mathrm{H} \alpha$ emitters discovered here, are presented in Giesers et al. (2019).

\subsection{Pulsating variables}

About $40 \%$ of all emission-line stars found in this survey are already known pulsating variable stars including W Viriginis variables, slow irregular variables, long-period variables, 


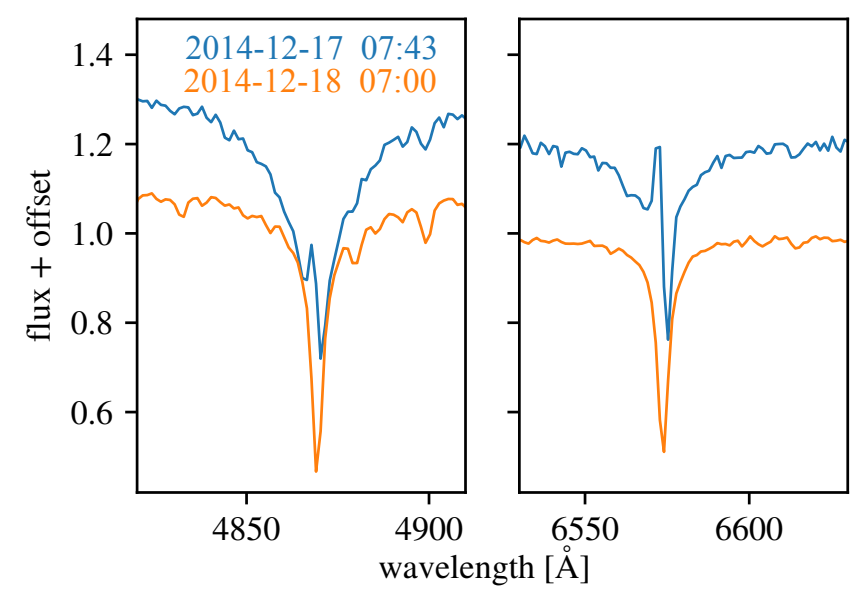

Fig. 7. Two spectra of the RR Lyrae star NGC 3201-V90 (CVSGGC) observed approximately $24 \mathrm{~h}$ apart show very different Balmer profiles. $\mathrm{H} \alpha$ and $\mathrm{H} \beta$ emission is only observed in one of the spectra, but not in the other one.

semiregular variables, and RR Lyrae variables. Spectra of these stars show constant or variable $\mathrm{H} \alpha$ and sometimes $\mathrm{H} \beta$ emission fluxes.

Since the work of Struve (1947) it is known that spectra of RR Lyrae stars have a varying, weak emission component in several Hydrogen lines. Figure 7 shows two spectra of the RR Lyrae star V90 in NGC 3201 as an example of variable emission lines. The two spectra were observed roughly $24 \mathrm{~h}$ apart and only the earlier one shows $\mathrm{H} \alpha$ and $\mathrm{H} \beta$ emission lines. The flux profiles are very similar to the first or second apparition of the RR Lyr variable X Ari shown Gillet \& Fokin (2014) in Fig. 1. The variable star V13 and possibly also V190 in NGC 6266 are currently classified as RR Lyrae stars in the literature. However, their spectra show very strong $\mathrm{H} \alpha$ and $\mathrm{H} \beta$ emission lines, similar to those of W Viriginis variables.

\subsection{Known nebulae}

There are four known planetary nebulae (PNe) in the whole globular cluster system of the Milky Way. These are Ps 1 in NGC 7078 (Pease 1928), GJJC-1 in NGC 6656 (Gillett et al. 1989), JaFu-1 in Pal 6, and JaFu-2 in NGC 6441 (both Jacoby et al. 1997).

The most successful and also largest survey of PNe in globular clusters is the one from Jacoby et al. (1997), who used the on-band/off-band technique at the [O III] line at $5007 \AA$ on 133 globular clusters. With this survey, they doubled the number of known PNe in GCs from two to four.

Of the four globular clusters with known PNe, Pal 6 is not included in our sample of GCs. Although our survey covers NGC 6656, GJJC-1 is not inside the MUSE FoV because of its position inside the cluster. This leaves Ps 1 and JaFu- 2 for which we provide flux maps and spectra. Figure 8 shows [O III] maps of the two PNe side-by-side, the PN spectra are shown in Fig. 9. The spectra were extracted with a relatively large circular aperture covering the whole nebula. Although the nebulae are not HST point sources and, accordingly, we did not automatically extract a spectrum at their positions, the detection algorithm finds the nebular emission lines. This is because their emission flux contaminated the otherwise purely stellar spectra of dozens of nearby stars as its high spatial variability is not accounted for in the extraction.
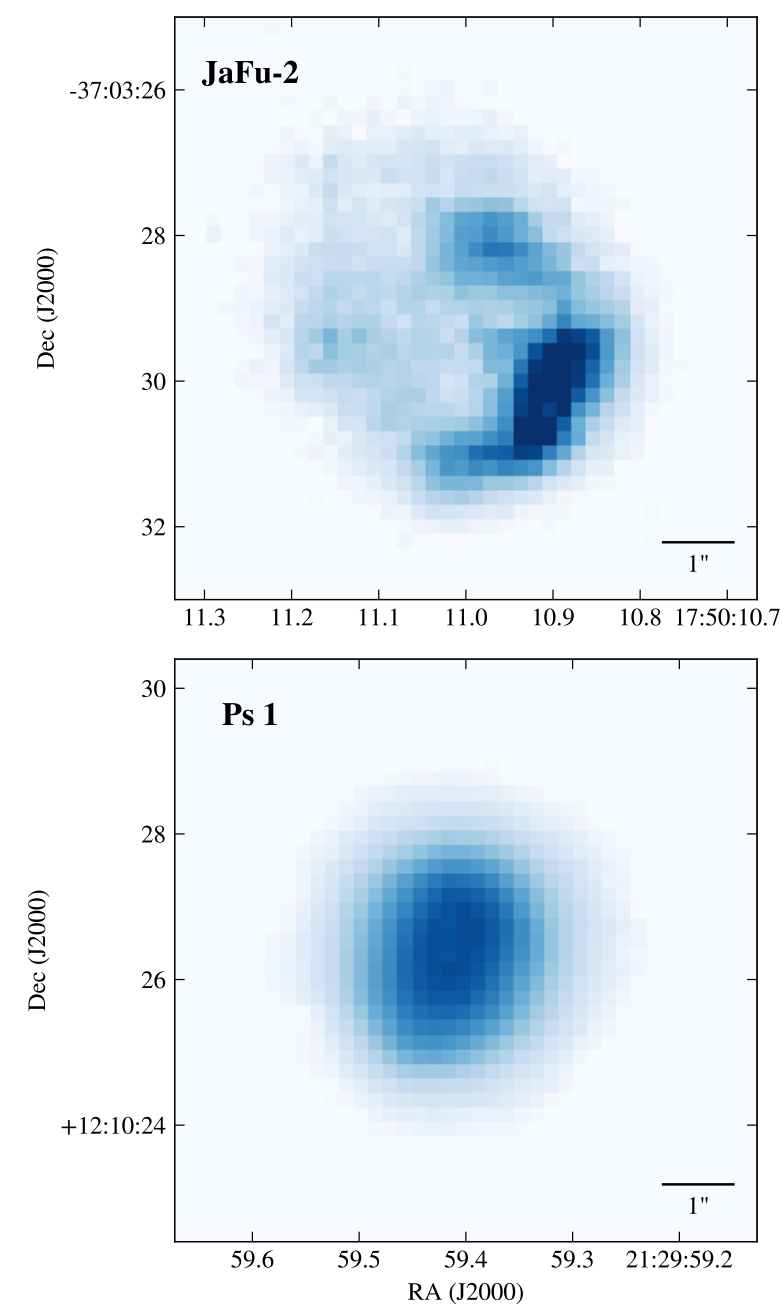

Fig. 8. Flux maps of the two known planetary nebulae in our sample: JaFu-2 (top) in NGC 6441 and Ps 1 (bottom) in NGC 7078. Shown is the $[\mathrm{O}$ III $] \lambda 5007$ flux after the stellar background has been subtracted.

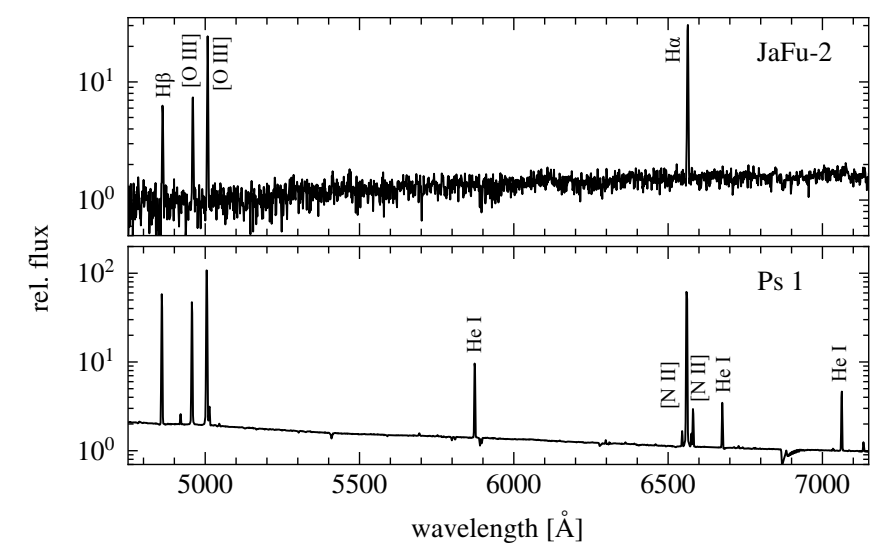

Fig. 9. Spectra of JaFu-2 (top) and Ps 1 (bottom). Prominent emission lines of Hydrogen, He I, [N II] and [O III] are labelled.

In addition to these two planetary nebulae, we detected a nova remnant in NGC 6656 which is described in detail in Göttgens et al. (2019). However, we did not find any additional nebulae in our observations. We checked this null-result by stacking cubes from different observations to increase our sensitivity. We used the on-band/off-band technique around $\mathrm{H} \alpha$ to search for extended emitting regions and did not find any nebula. 
Table 3. Positions and redshifts of background galaxies with emission lines.

\begin{tabular}{lcccc}
\hline \hline Cluster & RA $\left[^{\circ}\right]$ & $\operatorname{Dec}\left[{ }^{\circ}\right]$ & $d_{\mathrm{C}}\left[^{\prime \prime}\right]$ & $z$ \\
\hline NGC 104 & 5.97861 & -72.10556 & 100.6 & 0.330 \\
NGC 104 & 6.05634 & -72.05887 & 88.4 & 0.472 \\
NGC 1851 & 78.52291 & -40.03877 & 31.5 & 0.309 \\
NGC 1851 & 78.53530 & -40.03363 & 50.5 & 0.633 \\
NGC 5139 & 201.66150 & -47.40166 & 293.4 & 0.144 \\
NGC 5139 & 201.66193 & -47.39549 & 314.4 & 0.052 \\
NGC 5904 & 229.61804 & 2.06133 & 102.0 & 0.414 \\
NGC 6266 & 255.29045 & -30.10210 & 58.0 & 0.722 \\
NGC 6388 & 264.07237 & -44.73578 & 1.8 & 0.420 \\
NGC 6541 & 272.02490 & -43.70413 & 55.1 & 0.413 \\
NGC 6681 & 280.79848 & -32.28750 & 21.9 & 0.305 \\
NGC 6681 & 280.79929 & -32.29805 & 24.4 & 0.173 \\
NGC 6752 & 287.66226 & -59.98960 & 100.4 & 0.364 \\
NGC 6752 & 287.69412 & -59.96586 & 79.0 & 0.312 \\
NGC 6752 & 287.69667 & -59.96068 & 93.5 & 0.246 \\
NGC 6752 & 287.72543 & -60.01487 & 110.1 & 0.505 \\
NGC 6752 & 287.77154 & -59.98476 & 98.0 & 0.105 \\
NGC 7078 & 322.49858 & 12.17520 & 35.4 & 0.261 \\
NGC 7078 & 322.50275 & 12.15647 & 51.0 & 0.670 \\
NGC 7089 & 323.37114 & -0.82728 & 34.0 & 0.737 \\
NGC 7099 & 325.09295 & -23.17880 & 4.6 & 0.398 \\
\hline
\end{tabular}

Notes. The projected angular separation to the cluster centre is $d_{\mathrm{C}}$.

\subsection{Galaxies}

For each spectrum, we used the full list of emission-line candidates and their wavelengths to check if they correspond to a list of typical galactic emission lines, assuming they all have the same redshift. Using this method, we find 21 background galaxies that contaminate spectra we have extracted at known stellar positions in observations of several GCs (see Table 3). Since these spectra contain the stellar $\mathrm{H} \alpha$ absorption line, we conclude that these are indeed blended spectra of a star and a background galaxy. The spectra were identified by their prominent emission lines of Hydrogen and ionised Oxygen, as shown in Fig. 10 for three examples. We detected emission lines corresponding to restframe wavelengths of $3727 \AA$ from [O II], of $4959 \AA$ and $5007 \AA$ from [O III], and $\mathrm{H} \beta$ and $\mathrm{H} \alpha$ emission. Table 3 lists the position, the redshift calculated from the emission lines, and the projected angular separation to the cluster centre for each galaxy. Because of the low [N II] to $\mathrm{H} \alpha$ flux, all galaxies fall into the region occupied by starburst galaxies in the BPT diagram (Baldwin et al. 1981; Veilleux \& Osterbrock 1987), except for one galaxy behind NGC 6752 at $z=0.364$ which lies at the border of Seyfert galaxies and LINER. Deeper photometric observations of the fields containing the new galaxies may identify the counterparts which could be used to convert relative stellar proper motions into absolute proper motions, as done for NGC 6681 using HST images (Massari et al. 2013). These serendipitous discoveries resemble the one reported by Bedin et al. (2019), who found a dwarf spheroidal galaxy behind the globular cluster NGC 6752 using HST photometry. The fact that a galaxy lies very close to the core of the core-collapsed cluster NGC 7099 shows our capability to look through the GCs.

\subsection{Unidentified sources}

We find several emission-line stars that lie close to known X-ray sources. As a reference, we used the November 2017 pre-release of the Chandra Source Catalog Release 2.0 (Evans et al. 2010), which includes positions and error ellipses (including astrometric uncertainties) for X-ray sources in all but two clusters in our survey (NGC 6254 and NGC 6624). Based on the source positions and the associated errors, we estimated that there is no physical relation between a star and an X-ray source if their distance is $>1^{\prime \prime}$. In general, multiple stars have a distance $>1^{\prime \prime}$ to an $\mathrm{X}$-ray source which prohibits a unique identification of the optical counterpart. However, because both emission-line sources and X-ray sources are rare objects in globular clusters, we indicate if an X-ray source is close in the Table A.2.

As indicated in Table A.2, some stars show variable $\mathrm{H} \alpha$ emission wings or asymmetric absorption. In the case of giant stars, these features could point to chromospheric activity or mass motions (e.g. Cohen 1976; Cacciari et al. 2004; Meszaros et al. 2008).

\section{Discussion and conclusions}

\subsection{Completeness of the extraction of stellar sources}

There are two steps in the detection of emission-line sources in MUSE data that influence how many existing sources can be found: the extraction completeness and the efficiency of matched filtering. We discuss only the first one here, the second one was described in Sect. 3.3.

The extraction completeness is the ratio of ACS catalogue sources for which a spectrum can be extracted from the MUSE datacube to all sources in the MUSE field of view. Figure 11 shows the dependence of our extraction completeness for all clusters taking all spectra with a $\mathrm{S} / \mathrm{N}$ better than five into account. An extraction completeness of $100 \%$ does not mean that we have a spectrum of all stars in our FoV but only of those listed in the ACS catalogue. This is an important distinction for low brightness stars in the central few arcseconds of core-collapsed clusters, such as NGC 7078. Thus we expect that the completeness depends not only on the brightness of a star but also on its position relative to the cluster centre (see Fig. A.1).

We estimate the extraction completeness for different magnitudes and for three regions: the whole FoV, the central 10", the intermediate region from $10^{\prime \prime}$ to $60^{\prime \prime}$ and the remaining outer regions. Figure A.1 shows how our extraction completeness depends on the brightness of the star and its position in the case of NGC 7078. In general we see a completeness of close to $100 \%$ for bright stars throughout the cluster. The crowded cluster centre hinders the extraction of faint sources and the completeness starts to drop below $50 \%$ for magnitudes between 18 and 19 mag for most clusters.

\subsection{Do we find enough CVs?}

Massive globular clusters are expected to host about 200 CVs (Ivanova et al. 2006; Knigge 2012). However, the cluster with the most $\mathrm{CV}$ candidates, as determined by $\mathrm{UV}$ and optical photometry and X-ray data, is NGC 104 with 43 CVs (Rivera Sandoval et al. 2018). In contrast, the number of spectroscopically confirmed CVs is much lower: only ten CVs have been confirmed by spectroscopy in the whole globular cluster system of the Milky Way (Knigge 2012; Webb \& Servillat 2013). We add seven CVs to this list, including two newly detected CVs. CVs in globular clusters are hard to observe by spectroscopy because of the low brightness of the secondary component and crowding. We expect to find dwarf novae (DNe), a subtype of CVs, because they have spectra with emission 


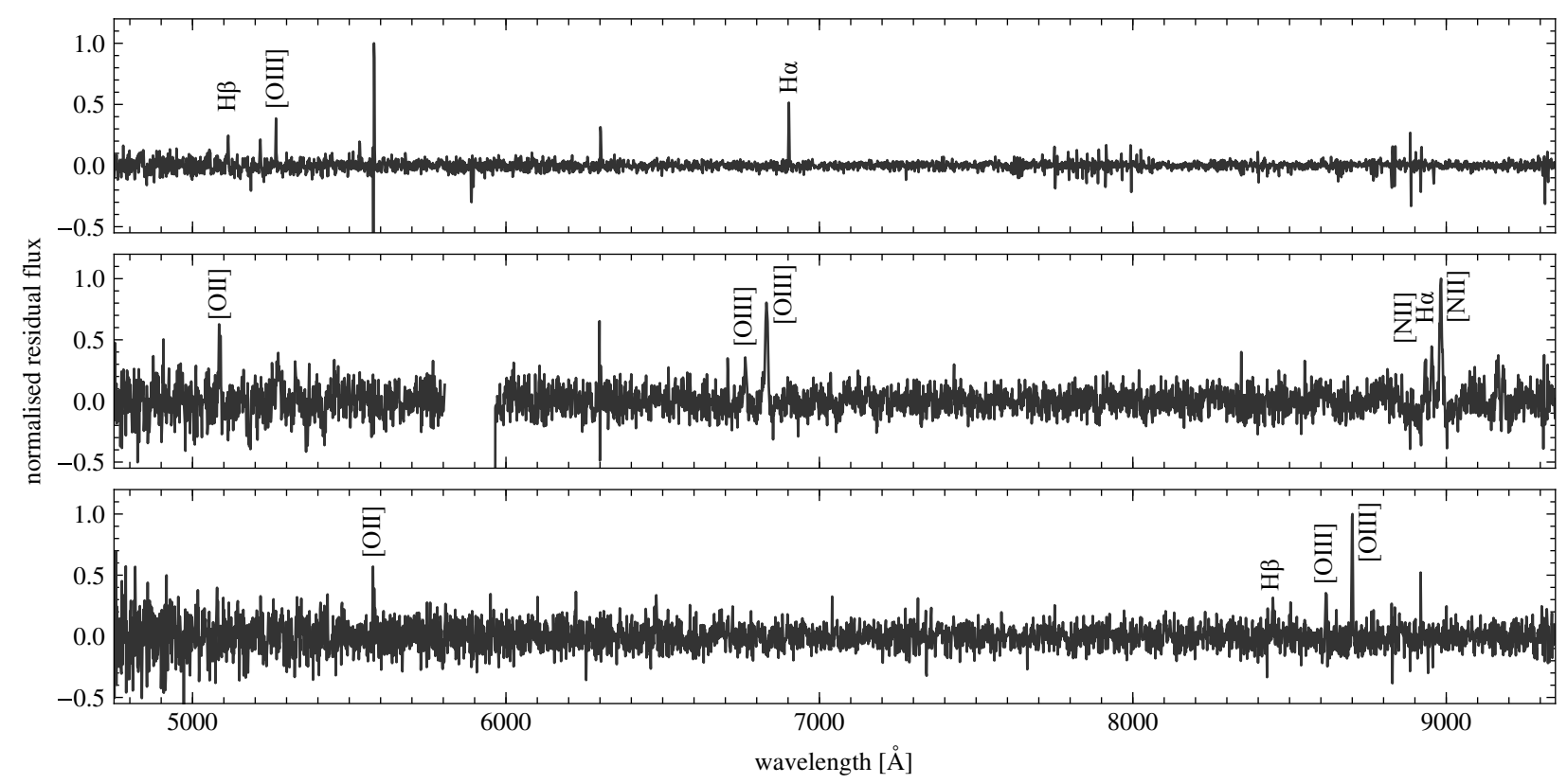

Fig. 10. Normalised residuals of stellar spectra that contain extragalactic emission lines including from [O II], [O III], and H I. Redshifts determined from these lines are $z=0.05,0.36$ and 0.737 , respectively. Top and bottom panels: starburst galaxy, while middle panel: AGN.

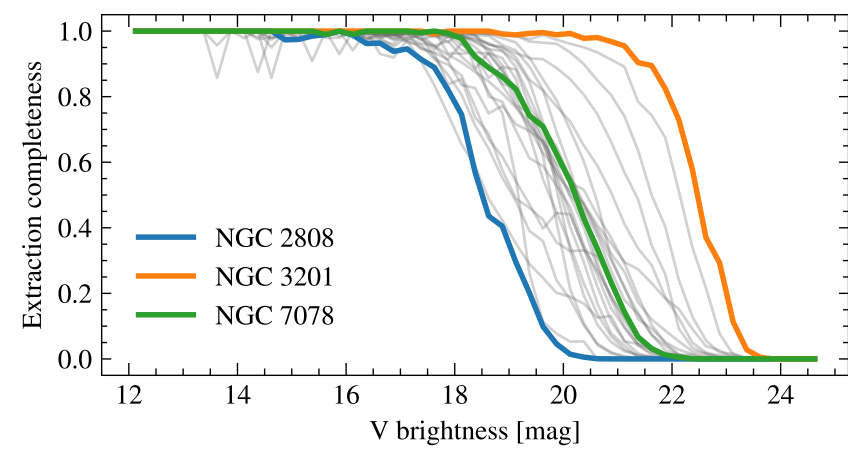

Fig. 11. Spectral extraction completeness $(S / N>5)$ for all clusters with respect to the ACS catalogue as a function of brightness (filters are listed in Table 4). Each grey curve represents a single cluster, the curves for NGC 2808, NGC 3201, and NGC 7078 are highlighted. Since the completeness mainly depends on the stellar density, it also depends on the radial distance to the cluster centre (see Fig. A.1).

lines in quiescence (Clarke et al. 1984; Warner 1995) and observations show that most CVs are DNe (Knigge et al. 2011). Is our number of $\mathrm{CV}$ detections consistent with the prediction? To answer this question, we used the average CV brightness distribution from MOCCA simulations of globular clusters (Belloni et al. 2016). We did not consider the effects of incomplete spatial coverage in our survey, because most CVs are expected to be located inside the half-mass radius of the respective cluster (Belloni et al. 2016, but also see Belloni et al. 2019). For each cluster, we drew samples from this distribution and used our completeness function and a detection probability of $80 \%$ (Sect. 3.3) to estimate the number of CVs for which we should have extracted spectra (see Table A.1). Since the clusters differ in their structural parameters and Belloni et al. (2016) give a $\mathrm{CV}$ brightness distribution for an average globular cluster, the number of CVs for each individual cluster is probably not meaningful. The total number of expected CV detections using the model of Belloni et al. (2016) in our sample is $10 \pm 2$, which is consistent with our number of nine detected CVs.
Table 4. Choice of $V$ filter for each cluster used in this paper.

\begin{tabular}{ll}
\hline \hline ACS/WFC3 filter & Clusters \\
\hline F625W & NGC 6522 \\
F555W & NGC 1904, NGC 6266, NGC 6293 \\
F606W & NGC 104, NGC 1851, NGC 2808, NGC 3201 \\
& NGC 362, NGC 5139, NGC 5286, NGC 5904, \\
& NGC 6093, NGC 6121, NGC 6218, NGC 6254, \\
& NGC 6388, NGC 6441, NGC 6541, NGC 6624 \\
& NGC 6656, NGC 6681, NGC 6752, NGC 7078 \\
& NGC 7089, NGC 7099 \\
\hline
\end{tabular}

The step that restricts the overall completeness for $\mathrm{CV}$ s the most is the extraction completeness at magnitudes of 22 and below. This could be improved with longer observations using the narrow-field mode (NFM) of MUSE, which will offer a much higher spatial sampling in a smaller FoV.

\subsection{Exclusion of more PNe}

While the limiting fluxes given in Table 2 are, if interpreted strictly, only valid for emission in stellar spectra, we can rule out any large diffuse source of $\mathrm{H} \alpha$ emission lines in our fields of view. Any nebula or other diffuse source of emission would need to overlap with at least some stars of which we extracted spectra. In the same way we easily detected JaFu-2, Ps 1 , and the nova remnant in NGC 6656 (Göttgens et al. 2019), these contaminated spectra would have been found.

There are still some possible but unlikely ways a hypothetical PNe could be hidden in a GC: It could be very small so that it only contaminates a few stars, ideally of low brightness. In this case, we might not extract a spectrum for them. The maximum size of this nebula cannot be large, considering the high density of sources for which we can extract spectra. Another possibility is a nebula with very faint $\mathrm{H} \alpha$ emission that would have to be much fainter than the known ones because those were easily found with our detection method. 
However, it is still possible that more nebulae similar to the nova in NGC 6656 lie outside the area covered by our survey. In this case, it has to be in a region of relatively high stellar density where it cannot be detected by photometric surveys.

\section{Summary}

We analysed data from our MUSE survey of 26 Galactic globular clusters, looking for signs of emission-line objects. Taking advantage of previous work on the same data, including data reduction, spectra extraction, and spectral analysis, we find 156 emission-line stars and several non-stellar emission-line sources. By assuming a Gaussian emission-line shape and using matched filtering, we detected this shape in the residuals generated during a full spectrum fit to the observed spectra. Since this generated many potentially interesting emission-line candidates, we use a threshold to select only the most promising candidates and checked them visually. We did not use external catalogues to search for known sources in our data, but we used them to validate and categorise our findings. We find two new cataclysmic variables, many known pulsating variable stars, and several unidentified emission-line stars close to known X-ray sources. The total number of $\mathrm{CVs}$ detected in this survey is consistent with numerical simulations when our spectral extraction completeness is taken into account. In addition to stellar emission-line sources, we also find 20 previously unknown starburst galaxies and one AGN in the background with redshifts from 0.05 to 0.74 .

Acknowledgements. We want to thank R. Terlevich for his helpful commen about the emission lines in the PN spectra. FG, SK, and SD acknowledge sup port from the German Research Foundation (DFG) through projects KA 4537/21 and DR 281/35-1. SK gratefully acknowledges funding from a European Research Council consolidator grant (ERC-CoG-646928- Multi-Pop). PMW SK, SD, and BG also acknowledge support from the German Ministry for Education and Science (BMBF Verbundforschung) through projects MUSE-AO, grants 05A14BAC and 05A14MGA, and MUSE-NFM, grants 05A17MGA and 05A17BAA. Based on observations made with ESO Telescopes at the La Silla Paranal Observatory under programme IDs 094.D-0142, 095.D-0629, 096.D0175, 097.D-0295, 098.D-0148, 099.D-0019, 0100.D-0161, 0101.D-0268, and 0102.D-0270. Also based on observations made with the NASA/ESA Hubble Space Telescope, obtained from the data archive at the Space Telescope Science Institute. STScI is operated by the Association of Universities for Research in Astronomy, Inc. under NASA contract NAS 5-26555.

\section{References}

Abbott, B., Abbott, R., Abbott, T., et al. 2016, Phys. Rev. Lett., 116, 061102 Albrow, M. D., Gilliland, R. L., Brown, T. M., et al. 2001, ApJ, 559, 1060 Anderson, J., Sarajedini, A., Bedin, L. R., et al. 2008, AJ, 135, 2055 Askar, A., Sedda, M. A., \& Giersz, M. 2018, MNRAS, 478, 1844 Bacon, R., Accardo, M., Adjali, L., et al. 2010, in Ground-based and Airborne Instrumentation for Astronomy III, Int. Soc. Opt. Photon., 7735, 773508

Bacon, R., Conseil, S., Mary, D., et al. 2017, A\&A, 608, A1 Baldwin, J. A., Phillips, M. M., \& Terlevich, R. 1981, PASP, 93, 5

Bassa, C., Pooley, D., Homer, L., et al. 2004, ApJ, 609, 755

Bedin, L. R., Salaris, M., Rich, R. M., et al. 2019, MNRAS, 484, L54

Belloni, D., Giersz, M., Askar, A., Leigh, N., \& Hypki, A. 2016, MNRAS, 462, 2950

Belloni, D., Giersz, M., Rivera Sandoval, L. E., Askar, A., \& Ciecielag, P. 2019, MNRAS, 483, 315

Cacciari, C., Bragaglia, A., Rossetti, E., et al. 2004, A\&A, 413, 343 Campos, F., Pelisoli, I., Kamann, S., et al. 2018, MNRAS, 481, 4397 Chomiuk, L., Strader, J., Maccarone, T. J., et al. 2013, ApJ, 777, 69 Clarke, J. T., Capel, D., \& Bowyer, S. 1984, ApJ, 287, 845 Clement, C. 2017, EPJ Web Conf., 152, 01021

Clement, C. M., Muzzin, A., Dufton, Q., et al. 2001, AJ, 122, 2587

Cohen, J. G. 1976, ApJ, 203, L127
Cool, A. M., Haggard, D., Arias, T., et al. 2013, ApJ, 763, 126 Dieball, A. 2008, Chandra Proposal 10300600

Dieball, A., Long, K. S., Knigge, C., Thomson, G. S., \& Zurek, D. R. 2010, ApJ, 710, 332

Evans, I. N., Primini, F. A., Glotfelty, K. J., et al. 2010, ApJS, 189, 37

Geller, A. M., Leiner, E. M., Bellini, A., et al. 2017a, ApJ, 840, 66 Geller, A. M., Leiner, E. M., Chatterjee, S., et al. 2017b, ApJ, 842, 1 Giesers, B., Dreizler, S., Husser, T.-O., et al. 2018, MNRAS, 475, L15

Giesers, B., Kamann, S., Dreizler, S., et al. 2019, A\&A, in press, https://doi.org/10.1051/0004-6361/201936203

Gillet, D., \& Fokin, A. B. 2014, A\&A, 565, A73

Gillett, F. C., Jacoby, G. H., Joyce, R. R., et al. 1989, ApJ, 338, 862 Göttgens, F., Weilbacher, P. M., Roth, M. M., et al. 2019, A\&A, 626, A69 Harris, W. E. 1996, AJ, 112, 1487

Heinke, C. O., Grindlay, J. E., Edmonds, P. D., et al. 2003, ApJ, 598, 516 Heinke, C. O., Grindlay, J. E., Edmonds, P. D., et al. 2005, ApJ, 625, 796 Herenz, E. C., \& Wisotzki, L. 2017, A\&A, 602, A111

Husser, T.-O., Wende-von Berg, S., Dreizler, S., et al. 2013, A\&A, 553, A6

Husser, T.-O., Kamann, S., Dreizler, S., et al. 2016, A\&A, 588, A148

Ivanova, N., Heinke, C. O., Rasio, F. A., et al. 2006, MNRAS, 372, 1043

Jacoby, G. H., Morse, J. A., Fullton, L. K., Kwitter, K. B., \& Henry, R. B. C. 1997, AJ, 114, 2611

Jacoby, G. H., Marco, O. D., Davies, J., et al. 2017, ApJ, 836, 93

Jeffreys, H. 1998, The Theory of Probability (Oxford: Oxford University Press)

Kaluzny, J., Rozyczka, M., Thompson, I. B., et al. 2016, Acta Astron., 66, 31

Kamann, S., Wisotzki, L., \& Roth, M. M. 2013, A\&A, 549, A71

Kamann, S., Husser, T.-O., Dreizler, S., et al. 2018, MNRAS, 473, 5591

Knigge, C. 2012, Mem. Soc. Astron. It., 83, 549

Knigge, C., Zurek, D. R., Shara, M. M., Long, K. S., \& Gilliland, R. L. 2003 , ApJ, 599, 1320

Knigge, C., Baraffe, I., \& Patterson, J. 2011, ApJS, 194, 28

Kremer, K., Ye, C. S., Chatterjee, S., Rodriguez, C. L., \& Rasio, F. A. 2018, ApJ, 855, L15

Kunder, A., Stetson, P. B., Catelan, M., Walker, A. R., \& Amigo, P. 2013, AJ, 145,33

Lebzelter, T., \& Wood, P. R. 2016, A\&A, 585, A111

Leiner, E., Mathieu, R. D., \& Geller, A. M. 2017, ApJ, 840, 67

Lu, T.-N., Kong, A. K. H., Bassa, C., et al. 2009, ApJ, 705, 175

Lugger, P. M., Cohn, H. N., Heinke, C. O., Grindlay, J. E., \& Edmonds, P. D. 2007, ApJ, 657, 286

Lugger, P. M., Cohn, H. N., Cool, A. M., Heinke, C. O., \& Anderson, J. 2017, ApJ, 841, 53

Massari, D., Bellini, A., Ferraro, F. R., et al. 2013, ApJ, 779, 81

Meszaros, S., Dupree, A. K., \& Szentgyorgyi, A. 2008, AJ, 135, 1117

Ochsenbein, F., Bauer, P., \& Marcout, J. 2000, A\&AS, 143, 23

Pease, F. G. 1928, PASP, 40, 342

Piotto, G., Milone, A. P., Bedin, L. R., et al. 2015, AJ, 149, 91

Pogson, N. 1860, MNRAS, 21, 32

Pooley, D. 2007, Chandra Proposal 09300101

Pooley, D., Lewin, W. H. G., Homer, L., et al. 2002, ApJ, 569, 405

Ransom, S. M. 2007, Proc. Int. Astron. Union, 3, 291

Rivera Sandoval, L. E., van den Berg, M., Heinke, C. O., et al. 2018, MNRAS, 475,4841

Roth, M. M., Sandin, C., Kamann, S., et al. 2018, A\&A, 618, A3

Sarajedini, A., Bedin, L. R., Chaboyer, B., et al. 2007, AJ, 133, 1658

Shara, M. M., \& Drissen, L. 1995, ApJ, 448, 203

Shishkovsky, L., Strader, J., Chomiuk, L., et al. 2018, ApJ, 855, 55

Soto, M., Bellini, A., Anderson, J., et al. 2017, AJ, 153, 19

Strader, J., Chomiuk, L., Maccarone, T. J., Miller-Jones, J. C. A., \& Seth, A. C. 2012, Nature, 490, 71

Struve, O. 1947, PASP, 59, 192

Veilleux, S., \& Osterbrock, D. E. 1987, ApJS, 63, 295

Vio, R., \& Andreani, P. 2016, A\&A, 589, A20

Warner, B. 1995, Cataclysmic Variable Stars, Cambridge Astrophysics (Cambridge, UK: Cambridge University Press), 126

Webb, N. A., \& Servillat, M. 2013, A\&A, 551, A60

Webb, N. A., Serre, D., Gendre, B., et al. 2004, A\&A, 424, 133

Weilbacher, P. M., Streicher, O., Urrutia, T., et al. 2012, in Software and Cyberinfrastructure for Astronomy II, Int. Soc. Opt. Photon., 8451, 84510B

Weilbacher, P. M., Streicher, O., Urrutia, T., et al. 2014, in Astronomical Data Analysis Software and Systems XXIII, eds. N. Manset, \& P. Forshay, ASP Conf. Ser., 485, 451

Wendt, M., Husser, T.-O., Kamann, S., et al. 2017, A\&A, 607, A133

Wenger, M., Ochsenbein, F., Egret, D., et al. 2000, A\&AS, 143, 9 


\section{Appendix A: Completeness}

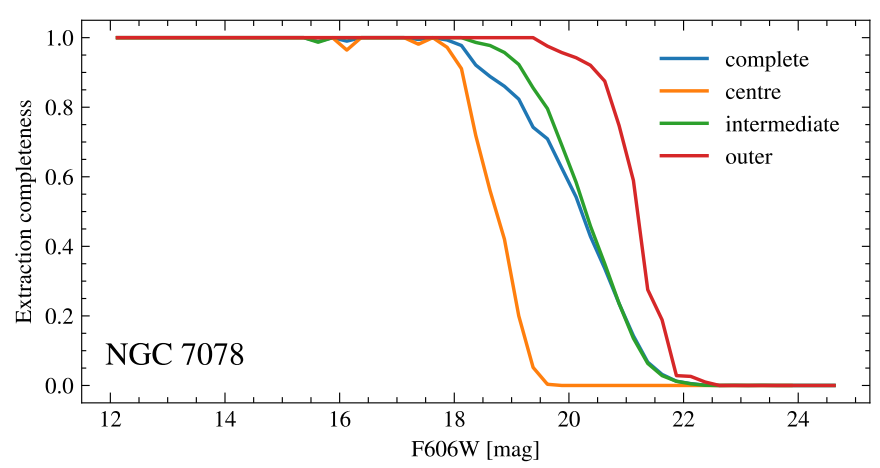

Fig. A.1. Spectral extraction completeness in NGC 7078 relative to the ACS catalogue. For this cluster, we reach a completeness of $50 \%$ at a magnitude of 17, 20, and 21.5 for the central region (innermost $10^{\prime \prime}$ ), an intermediate region (between $10^{\prime \prime}$ and $60^{\prime \prime}$ ), and the outer regions (outside $60^{\prime \prime}$ ), respectively.
Table A.1. Number of expected CVs in each cluster using the brightness distribution from Belloni et al. (2016).

\begin{tabular}{lcccc}
\hline \hline Cluster & Lower & Median & Upper & $N_{\text {found }}$ \\
\hline NGC 3201 & 1.5 & 2.9 & 4.8 & \\
NGC 6218 & 0.9 & 1.7 & 3.3 & 1 \\
NGC 6254 & 0.8 & 1.6 & 2.9 & \\
NGC 6656 & 0.5 & 1.3 & 2.1 & 1 \\
NGC 6752 & 0.4 & 0.9 & 1.4 & 1 \\
NGC 6121 & 0.1 & 0.3 & 0.9 & \\
NGC 104 & 0.0 & 0.1 & 0.3 & 1 \\
NGC 5904 & 0.0 & 0.1 & 0.3 & \\
NGC 6681 & 0.0 & 0.1 & 0.3 & 1 \\
NGC 7099 & 0.0 & 0.1 & 0.6 & 1 \\
NGC 6266 & 0.0 & 0.0 & 0.2 & \\
NGC 6624 & 0.0 & 0.0 & 0.2 & \\
NGC 5139 & 0.0 & 0.0 & 0.2 & 1 \\
NGC 6541 & 0.0 & 0.0 & 0.1 & \\
NGC 5286 & 0.0 & 0.0 & 0.0 & \\
NGC 6388 & 0.0 & 0.0 & 0.0 & \\
NGC 6441 & 0.0 & 0.0 & 0.0 & \\
NGC 7078 & 0.0 & 0.0 & 0.0 & \\
NGC 6093 & 0.0 & 0.0 & 0.0 & 2 \\
NGC 1851 & 0.0 & 0.0 & 0.0 & \\
NGC 6293 & 0.0 & 0.0 & 0.0 & \\
NGC 6522 & 0.0 & 0.0 & 0.0 & \\
NGC 362 & 0.0 & 0.0 & 0.0 & \\
NGC 2808 & 0.0 & 0.0 & 0.0 & \\
NGC 1904 & 0.0 & 0.0 & 0.0 & \\
NGC 7089 & 0.0 & 0.0 & 0.0 & \\
Total & 8.1 & 10.0 & 12.5 & 9 \\
\hline
\end{tabular}

Notes. Columns "lower" and "upper" give the $1 \sigma$ interval around the median value listed in column "median". The column $N_{\text {found }}$ refers to the number of spectroscopically detected CVs in our survey. 


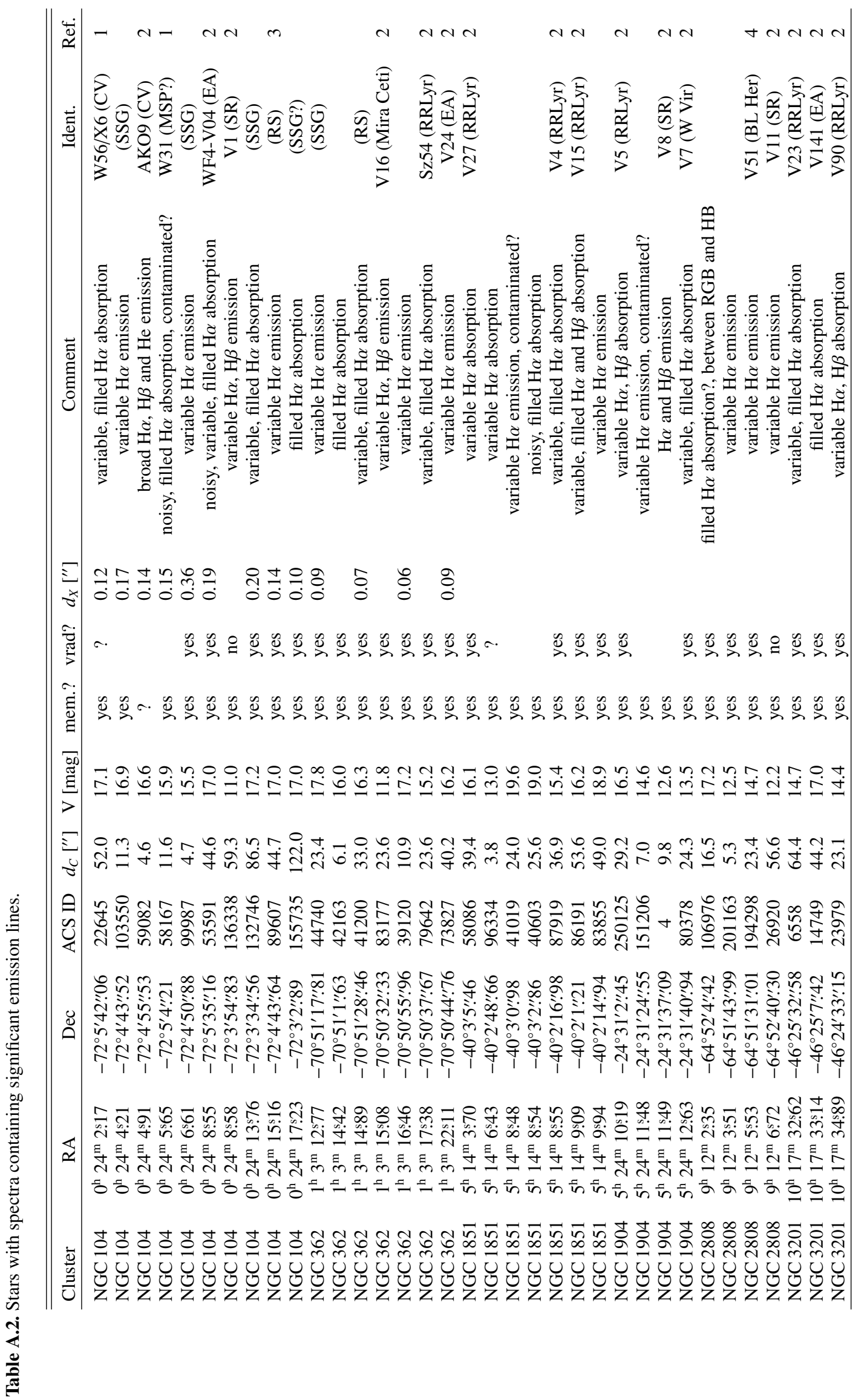

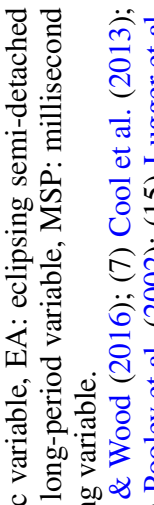

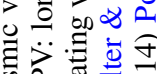

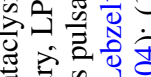

J.

30

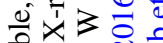

每

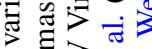

on $30 \overline{0}$

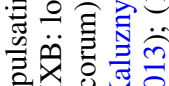

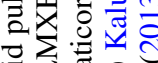

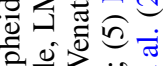

क्ष

等

o

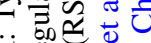

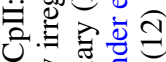

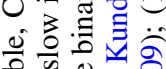

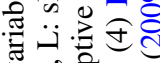

6n

.

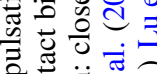

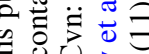

可参论

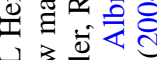

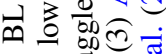

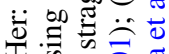

슬

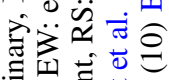

o

急言要

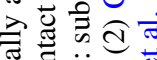

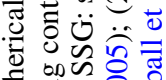

政.

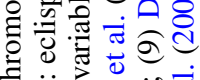

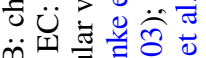

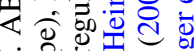

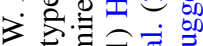

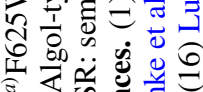

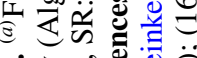

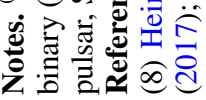

A118, page 13 of 16 


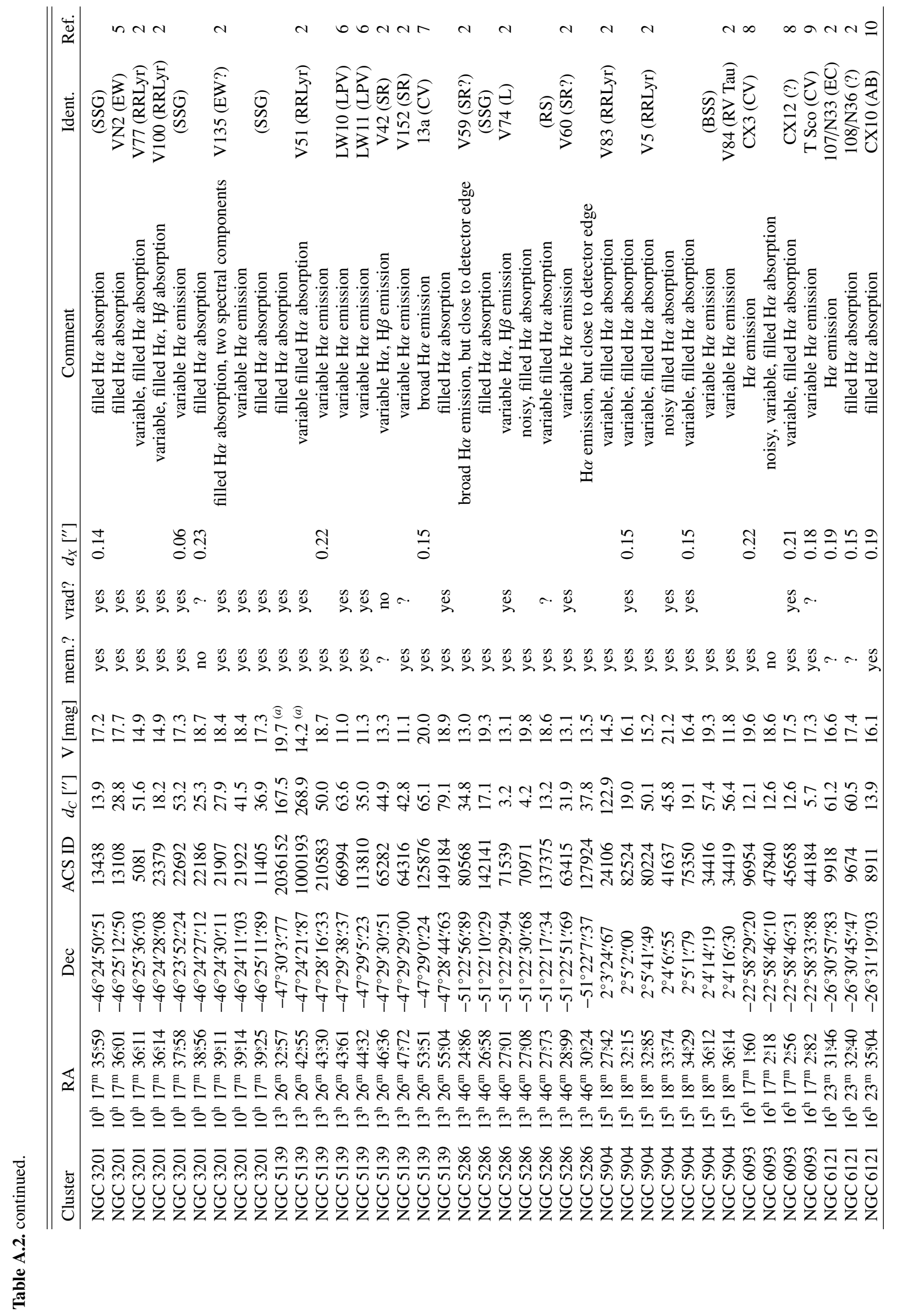


F. Göttgens et al.: A spectral catalogue of emission-line sources in Galactic globular clusters

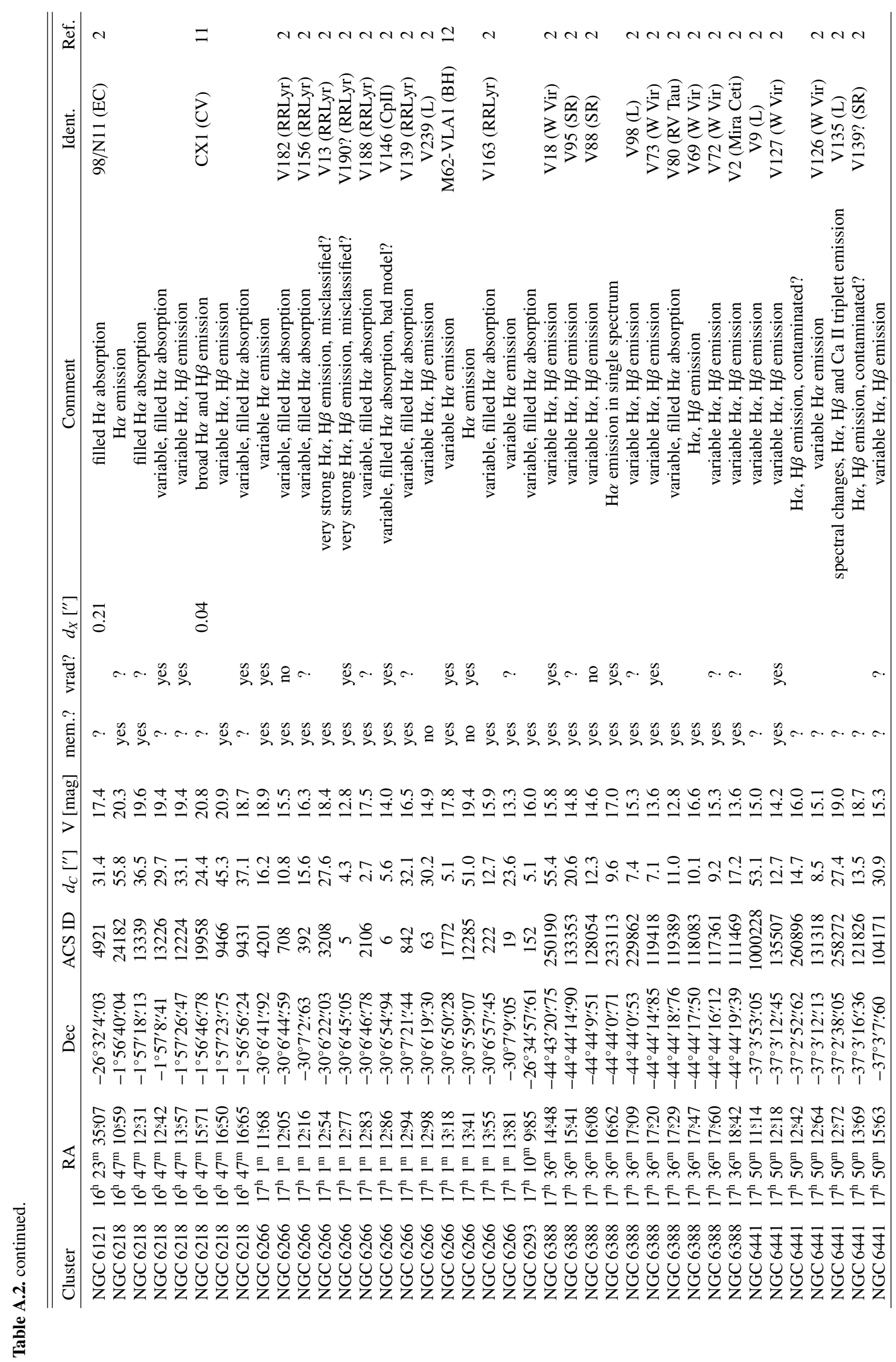




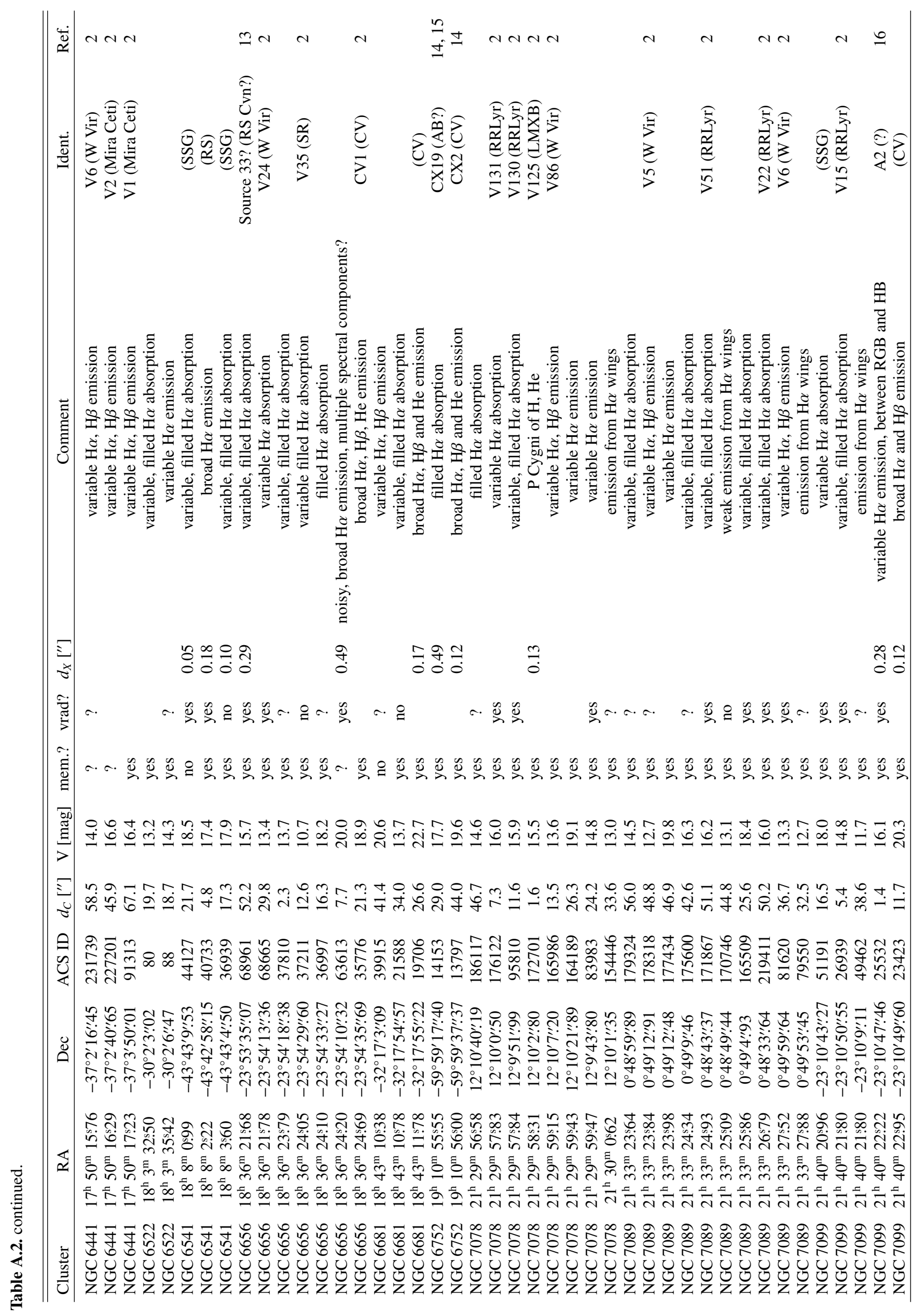

